\title{
Quiver Gauge Theory and Conformality at the TeV Scale
}

\author{
Paul H. Frampton ${ }^{(a)}$ and Thomas W. Kephart ${ }^{(b)}$ \\ (a) Department of Physics and Astronomy, University of North Carolina, \\ Chapel Hill, NC 27599. \\ (b) Department of Physics and Astronomy, Vanderbilt University, \\ Nashville, TN 37235.
}

\begin{abstract}
This review describes the conformality approach to extending the standard model of particle phenomenology using an assumption of no conformal anomaly at high energy. Topics include quiver gauge theory, the conformality approach to phenomenology, strong-electroweak unification at $4 \mathrm{TeV}$, cancellation of quadratic divergences, cancellation of $\mathrm{U}(1)$ anomalies, and a dark matter candidate.
\end{abstract}




\section{Table of Contents}

\section{Introduction}

1.1 Beyond the standard model

2. Quiver gauge theories

2.1 Orbifolding

\section{Conformality phenomenology}

3.1 Experimental evidence for conformality.

4. Tabulation of simplest abelian quivers

5. Chiral fermions

6. Model building

6.1 Abelian model building

6.1.1 Abelian non-SUSY models

6.1.2 $\mathcal{N}=1$ chiral $Z_{n}$ models.

6.1.3 $\mathcal{N}=0,1$ chiral models for abelian product groups.

6.1.4 Abelian SUSY models.

6.2 Non abelian model building

6.2.1 Non-Abelian non-SUSY models

6.3 Non-abelian groups with order $g \leq 31$

6.3.1 The Scalar Sector

6.3.2 Spontaneous symmetry breaking

\section{Unification}

7.1 Grand unification at $4 \mathrm{TeV}$.

8. Quadratic divergences

8.1 Is there a global symmetry?

9. U(1) Factors

9.1 Conformality of $\mathrm{U}(1)$ couplings.

\section{Dark matter candidate}

\section{Proton decay}

\section{Conclusions}

Bibliography 


\section{Introduction}

The standard model of particle phenomenology is a gauge field theory based on the gauge group $S U_{C}(3) \times S U_{L}(2) \times U_{Y}(1)$ and with three families of quarks and leptons. The electroweak symmetry $S U_{L}(2) \times U_{Y}(1)$ is spontaneously broken by a Higgs mechanism to the electromagnetic symmetry $U_{E M}(1)$, leaving one Higgs boson.

This model has successfully explained all experimental data (with the exception of neutrino masses which can be accommodated by extension). At higher energy than yet explored, the proliferation of parameters (19 without neutrino mass, 28 with) strongly suggests new physics beyond the standard model.

In this review we discuss such an extension based on four dimensional conformal invariance at high energy and inspired initially by the duality between gauge theory and superstring theory.

Such conformality model building is a less explored but equally motivated alternative to other directions of model building such as extra dimensions or supersymmetry.

\subsection{Beyond the standard model}

Particle phenomenology is in an especially exciting time, mainly because of the anticipated data in a new energy regime expected from the Large Hadron Collider (LHC), to be commissioned at the CERN Laboratory in 2007. This new data is long overdue. The Superconducting Supercollider (SSC) could have provided such data long ago were it not for its political demise in 1993.

Except for the remarkable experimental data concerning neutrino masses and mixings which has been obtained since 1998, particle physics has been data starved for the last thirty years. 
The standard model invented in the '60s and '70s has been confirmed and reconfirmed. Consequently, theory has ventured into speculative areas such as string theory, extra dimensions and supersymmetry. While these ideas are of great interest and theoretically consistent there is no direct evidence from experiment for them. Here we describe a more recent, post 1998, direction known as conformality. First, to set the stage, we shall discuss why the conformality approach which is, in our opinion, competitive with the other three approaches, remained unstudied for the twenty years up to 1998 .

A principal motivation underlying model building, beyond the standard model, over the last thirty years has been the hierarchy problem which is a special case of naturalness. This idea stems from Wilson [1] in the late '60s. The definition of naturalness is that a theory should not contain any unexplained very large (or very small in the inverse) dimensionless numbers. The adjustment needed to achieve such naturalness violating numbers is called fine tuning. The naturalness situation can be especially acute in gauge field theories because even after fine tuning at tree level, i.e., the classical lagrangian, the fine tuning may need to be repeated an infinite number of times order by order in the loop expansion during the renormalization process. While such a theory can be internally consistent it violates naturalness. Thus naturalness is not only an aesthetic criterion but one which the vast majority of the community feel must be imposed on any acceptable extension of the standard model; ironically, one exception is Wilson himself [2].

When the standard model of Glashow [3] was rendered renormalizable by appending the Higgs mechanism $[4,5]$ it was soon realized that it fell into trouble with naturalness, specifically through the hierarchy problem. In particular, the scalar propogator has quadratically divergent radiative corrections whch imply that a bare Higgs mass $m_{H}^{2}$ will be corrected by an amount $\Lambda^{2} / m_{H}^{2}$ where $\Lambda$ is the cut off scale corresponding to new physics. 
Unlike logarithmic divergences, which can be absorbed in the usual renormalization process, the quadratic divergences create an unacceptable fine tuning: for example, if the cut off is at the conventional grand unification scale $\Lambda \sim 10^{16} \mathrm{GeV}$ and $m_{H} \sim 100 \mathrm{GeV}$, we are confronted with a preposterous degree of fine tuning to one part in $10^{28}$.

As already noted, this hierarchy problem was stated most forcefully by Wilson who said, in private discussions, that scalar fields are forbidden in gauge field theories. Between the late '60s and 1974, it was widely recognized that the scalar fields of the standard model created this serious hierarchy problem but no one knew what to do about it.

The next big progress to the hierarchy problem came in 1974 with the invention [6] of supersymmetry. This led to the Minimally Supersymmetric Standard Model (MSSM) which elegantly answered Wilson's objection since quadratic divergences are cancelled between bosons and fermions, with only logarithmic divergences surviving. Further it was proved $[7,8]$ that the MSSM and straightforward generalizations were the unique way to proceed. Not surprisingly, the MSSM immediately became overwhelmingly popular. It has been estimated [9] that there are 35,000 papers existing on supersymmetry, more than an average of one thousand papers per year since its inception. This approach continued to seem "unique" until 1998. Since the MSSM has over one hundred free parameters, many possiblities needed to be investigated and exclusion plots constructed. During this period, two properties beyond naturalness rendered the MSSM even more appealing: an improvement in unification properties and a candidate for cosmological dark matter.

Before jumping to 1998, it is necessary to mention an unconnected deveopment in 1983 which was the study of Yang-Mills theory with extended $\mathcal{N}=4$ supersymmetry (the MSSM has $\mathcal{N}=1$ supersymmetry). This remarkable theory, though phenomenologically quite unrealistic as it allows no chiral fermions 
and all matter fields are in adjoint representions, is finite [10-12] to all orders of perturbation theory and conformally invariant. Between 1983 and 1997, the relationship between the $\mathcal{N}=4$ gauge theory and either string theory, also believed to be finite, or the standard model remained unclear.

The perspective changed in 1997-98 initially through the insight of Maldacena [13] who showed a duality between $\mathcal{N}=4$ gauge theory and the superstring in ten spacetime dimensions. Further the $\mathcal{N}=4$ supersymmetry can be broken by orbifolding down to $\mathcal{N}=0$ models with no supersymmetry at all. It was conjectured [14] by one of the authors in 1998 that such nonsupersymmetric orbifolded models can be finite and conformally invariant, hence the name conformality.

Conformality models have been investigated far less completely than supersymmetric ones but it is already clear that supersymmetry is "not as unique" as previously believed. Nogo theorems can have not only explicit assumptions which need to be violated to avoid the theorem but unconcious implicit assumptions which require further progress even to appreciate: in 1975 the implicit assumption was that the gauge group is simple, or if semi-simple may be regarded as a product of theories each with a simple gauge group. Naturalness, by cancellation of quadratic divergences, accurate unification and a dark matter candidate exist in conformality.

It becomes therefore a concern that the design of the LHC has been influenced by the requirement of testing the MSSM. The LHC merits an investment of theoretical work to check if the LHC is adequately designed to test conformality which now seems equally as likely as supersymmetry, although we fully expect the detectors ATLAS and CMS to be sufficiently all purpose to capture any physics beyond the standad model at the TeV scale. 


\section{Quiver gauge theories}

Quiver gauge theories possess a gauge group which is generically a product of $U\left(N_{i}\right)$ factors with matter fields in bifundamental representations. They have been studied in the physics literature since the 1980s where they were used in composite model building. They have attracted much renewed attention because of their natural appearance in the duality between superstrings and gauge theories.

The best known such duality gives rise to a highly supersymmetric $(\mathcal{N}=4)$ gauge theory with a single $S U(N)$ gauge group with matter in adjoint representations. In this case one can drop with impunity the $U(1)$ of $U(N)$ because the matter fields are uncharged under it. In the quiver theories with less supersymmetry $(\mathcal{N} \leq 2)$ it is usually necessary to keep such $U(1) \mathrm{s}$.

Quiver gauge theories are taylor made for particle physics model building. While an $S U(N)$ gauge theory is typically anomalous in for arbitrary choice of fermions, choosing the fermions to lie in a quiver insures anomaly cancelation. Furthermore the fermions in a quiver arrange themselves in bifundamental representations of the product gauge group. This nicely coinsides with the fact that all known fundamental fermions are in bifundamental, fundamental, or singlet representations of the gauge group. The study of quiver gauge theories goes back to the earliest days of gauge theories and the standard model. Other notable early examples are the Pati-Salam model and the trinification model. A vast literature exists on this subject, but we will concentrate on post $A d S / C F T$ conjecture quiver gauge theory work [15-139].

Starting from $A d S_{5} \times S^{5}$ we only have an $S U(N) \mathcal{N}=4$ supersymmetric gauge theory. In order to break SUSY and generate a quiver gauge theory there are several options open to us. Orbifolds [140-143], conifolds [144-148], and orientifolds 
[149-154] have all played a part in building quiver gauge theories. Since our focus is quiver gauge theories in general, but via orbifolding of $A d S_{5} \times M^{5}$ in particular, we will not discuss the other options in detail but should point out that orbifolding from the eleven dimensional $M$ theory point of view has also an active area of research [155-160]. Furthermore, we are interested in orbifolds where the manifold $M^{5}$ is the five sphere $S^{5}$. There are other possible choices for $M^{5}$ of relevance to model building $[161,162]$ but we will not explore these here either. In building models from orbifolded $A d S_{5} \times S^{5}$, it is often convenient to break the quiver gauge group to the trinification [163] group $S U(3)^{3}$ or to the Pati-Salam [164-166] group $S U(4) \times S U(2) \times S U(2)$, but there are again other possibilities, including more complicated intermediate groups like the quartification [167-172] symmetry $S U(3)^{4}$ that treats quarks and leptons on an equal footing.

It is important to note that although the duality with superstrings is a significant guide to such model building, and it is desirable to have a string dual to give more confidence in consistency, we shall focus on the gauge theory description in the approach to particle phenomenology, as there are perfectly good quiver gauge theories that have yet to be derived from string duality.

\section{$2.1 \quad$ Orbifolding}

The simplest superstring - gauge duality arises from the compactifiation of a Type IIB superstring on the cleverly chosen manifold

$$
A d S_{5} \times S^{5}
$$

which yields an $\mathcal{N}=4$ supersymmetry which is an especially interesting gauge theory which has been intensively studied and 
possesses remarkable properties of finiteness and conformal invariance for all values of $N$ in its $S U(N)$ gauge group. By "conformality", we shall mean conformal invariance at high energy, also for finite $N$.

For phenomenological purposes, $\mathcal{N}=4$ is too much supersymmetry. Fortunately it is possible to breaking supersymmetries and hence approach more nearly the real world, with less or no supersymmetry in a conformality theory.

By factoring out a discrete (either abelian or nonabelian) group and composing an orbifold:

$$
S^{5} / \Gamma
$$

one may break $\mathcal{N}=4$ supersymmetry to $\mathcal{N}=2, \quad 1$, or $\quad 0$. Of special interest is the $\mathcal{N}=0$ case.

We may take an abelian $\Gamma=Z_{p}$ (non-abelian cases will also be considered in this review) which identifies $p$ points in a complex three dimensional space $\mathcal{C}_{3}$.

The rules for breaking the $\mathcal{N}=4$ supersymmetry are:

If $\Gamma$ can be embedded in an $S U(2)$ of the original $S U(4) \mathrm{R}$ symmetry, then

$$
\Gamma \subset S U(2) \quad \Rightarrow \quad \mathcal{N}=2 .
$$

If $\Gamma$ can be embedded in an $S U(3)$ but not an $S U(2)$ of the original $S U(4)$ R-symmetry, then

$$
\Gamma \subset S U(3) \quad \Rightarrow \quad \mathcal{N}=1 .
$$

If $\Gamma$ can be embedded in the $S U(4)$ but not an $S U(3)$ of the original $S U(4)$ R-symmetry, then

$$
\Gamma \subset S U(4) \quad \Rightarrow \quad \mathcal{N}=0 .
$$

In fact to specify the embedding of $\Gamma=Z_{p}$ we need to identify three integers $\left(a_{1}, a_{2}, a_{3}\right)$ : 


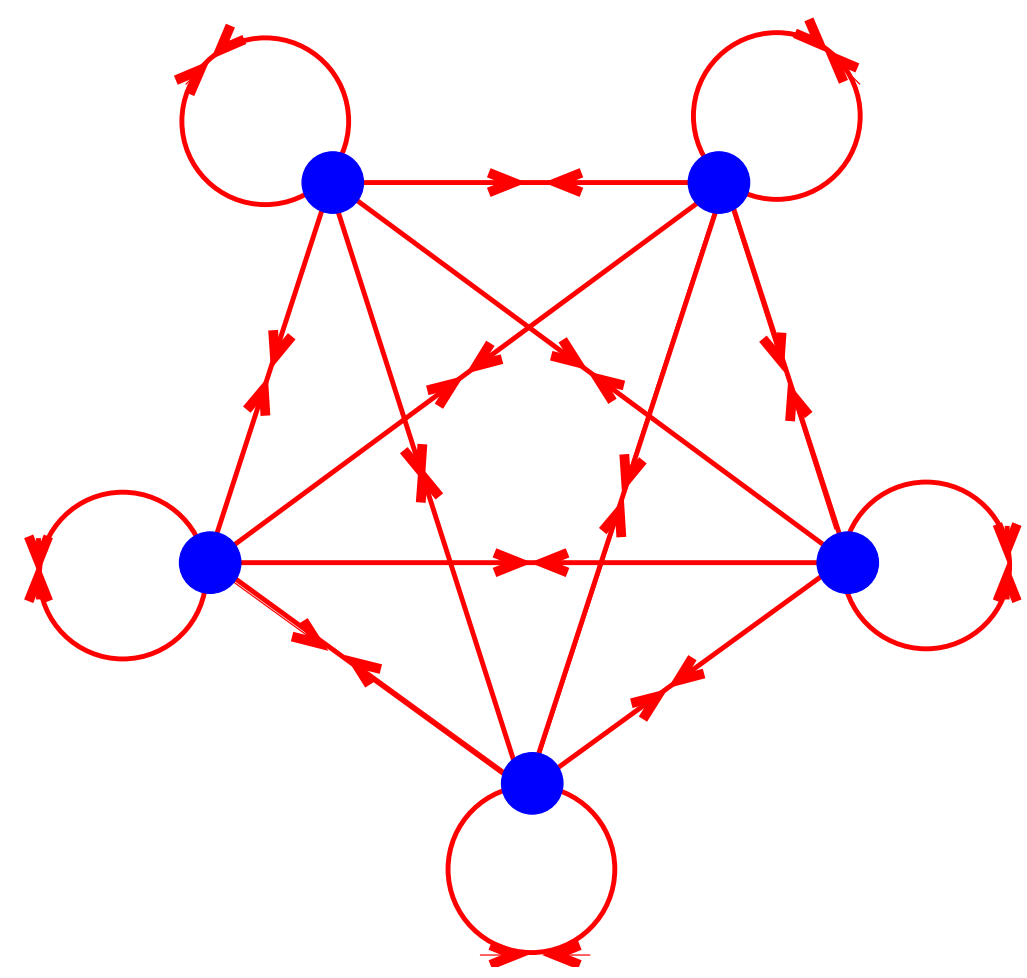

$$
\mathcal{C}_{3}: \quad\left(X_{1}, X_{2}, X_{3}\right) \stackrel{Z_{p}}{\rightarrow}\left(\alpha^{a_{1}} X_{1}, \alpha^{a_{2}} X_{2}, \alpha^{a_{3}} X_{3}\right)
$$

with

$$
\alpha=\exp \left(\frac{2 \pi i}{p}\right)
$$

The $Z_{p}$ discrete group identifies $p$ points in $\mathcal{C}_{3}$. The $\mathrm{N}$ converging D3-branes meet on all $p$ copies, giving a gauge group: $U(N) \times U(N) \times \ldots \ldots \times U(N), p$ times. The matter (spin-1/2 and spin-0) which survives is invariant under a product of a gauge transformation and a $Z_{p}$ transformation.

There is a convenient diagramatic way to find the result from a "quiver." One draws $p$ points and arrows for $a_{1}, a_{2}, a_{3}$.

Above is an example for $Z_{5}$ and $a_{i}=(1,3,0)$.

For a general case, the scalar representation contains the bifundamental scalars 


$$
\sum_{k=1}^{3} \sum_{i=1}^{p}\left(N_{i}, \bar{N}_{i \pm a_{k}}\right)
$$

Note that by definition a bifundamental representation transforms as a fundamental $\left(N_{i}\right)$ under $U(N)_{i}$ and simultaneously as an antifundamental $\left(\bar{N}_{i \pm a_{k}}\right)$ under $U(N)_{i \pm a_{k}}$.

For fermions, one must first construct the $\mathbf{4}$ of R-parity $S U(4)$, isomorphic to the isometry $S O(6)$ of the $S^{5}$. From the $a_{k}=$ $\left(a_{1}, a_{2}, a_{3}\right)$ one constructs the 4 -spinor $A_{\mu}=\left(A_{1}, A_{2}, A_{3}, A_{4}\right)$ :

$$
\begin{aligned}
& A_{1}=\frac{1}{2}\left(a_{1}+a_{2}+a_{3}\right) \\
& A_{2}=\frac{1}{2}\left(a_{1}-a_{2}-a_{3}\right) \\
& A_{3}=\frac{1}{2}\left(-a_{1}+a_{2}-a_{3}\right) \\
& A_{4}=\frac{1}{2}\left(-a_{1}-a_{2}+a_{3}\right)
\end{aligned}
$$

These transform as $\exp \left(\frac{2 \pi i}{p} A_{\mu}\right)$ and the invariants may again be derived (by a different diagram). An example of a fermion quiver with $p=5$ is shown above.

Note that these lines are oriented, as is necessary to accommodate chiral fermions. Specifying the four $A_{\mu}$ is equivalent (there is a constraint that the four add to zero, $\bmod p$ ) to fixing the three $a_{k}$ and group theoretically is more fundamental.

In general, the fermion representation contains the bifundamentals 


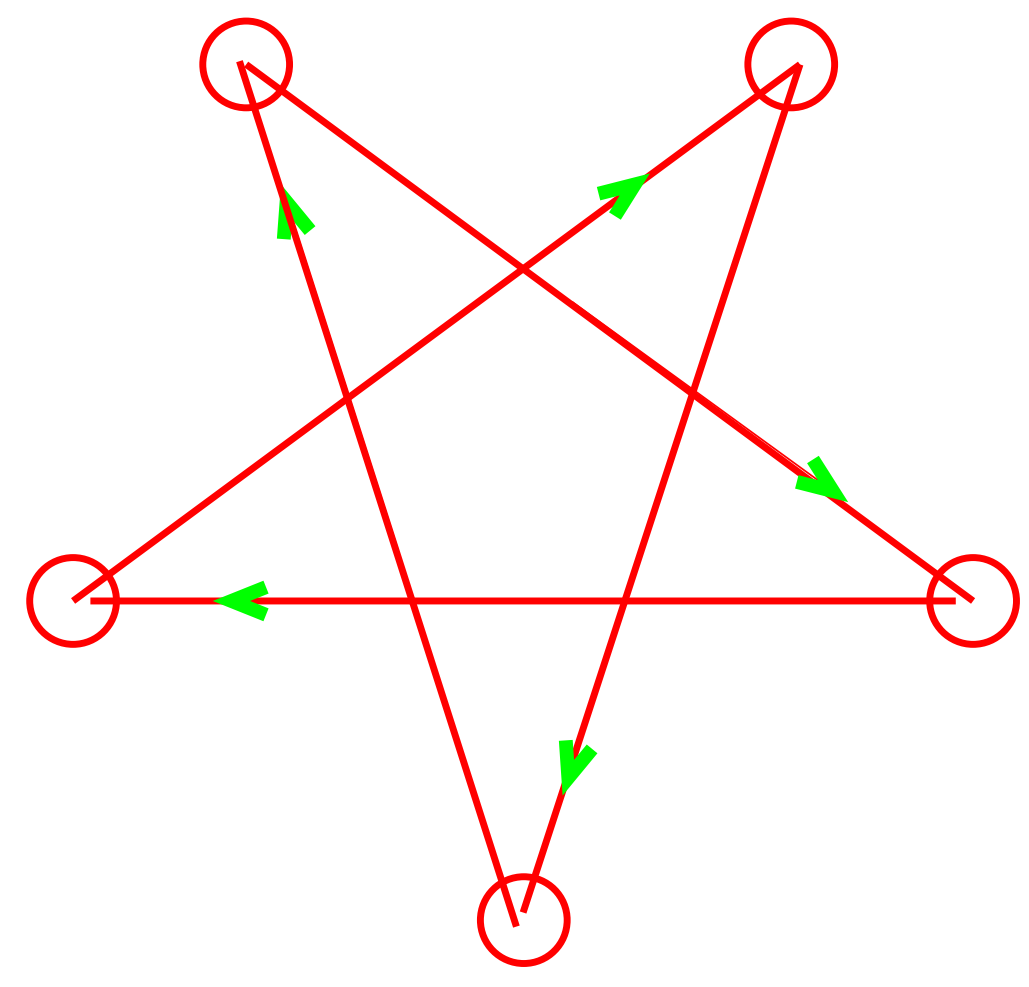

$$
\sum_{\mu=1}^{4} \sum_{i=1}^{p}\left(N_{i}, \bar{N}_{i+A_{\mu}}\right)
$$

When one of the $A_{\mu} \mathrm{s}$ is zero, it signifies a degenerate case of a bifundamental comprised of adjoint and singlet representations of one $U(N)$.

To summarize the orbifold construction, first we select a discrete subgroup $\Gamma$ of the $S O(6) \sim S U(4)$ isometry of $S^{5}$ with which to form the orbifold $A d S_{5} \times S^{5} / \Gamma$. As discussed above, the replacement of $S^{5}$ by $S^{5} / \Gamma$ reduces the supersymmetry to $\mathcal{N}=0,1$ or 2 from the initial $\mathcal{N}=4$, depending on how $\Gamma$ is embedded in the isometry of $S^{5}$. The cases of interest here are $\mathcal{N}=0$ and $\mathcal{N}=1 \quad S U S Y$ where $\Gamma$ embeds irreducibly in the $S U(4)$ isometry or in an $S U(3)$ subgroup of the $S U(4)$ isometry, respectively. I.e., to achieve $\mathcal{N}=0$ we embed $\operatorname{rep}(\Gamma) \rightarrow 4$ of $S U(4)$ as $\mathbf{4}=(\mathbf{r})$ where $\mathbf{r}$ is a nontrivial four dimensional rep- 
resentation of $\Gamma$; for $\mathcal{N}=1$ we embed $\operatorname{rep}(\Gamma) \rightarrow \boldsymbol{4}$ of $S U(4)$ as $\mathbf{4}=(\mathbf{1}, \mathbf{r})$ where $\mathbf{1}$ is the trivial irreducible representation (irrep) of $\Gamma$ and $\mathbf{r}$ is a nontrivial three dimensional representation of $\Gamma$. 


\section{Conformality phenomenology}

In attempting to go beyond the standard model, one outstanding issue is the hierarchy between GUT scale and weak scale which is 14 orders of magnitude. Why do these two very different scales exist? Also, how is this hierarchy of scales stabilized under quantum corrections? Supersymmetry answers the second question but not the first.

The idea is to approach hierarchy problem by Conformality at a TeV Scale. We will show how this is possible including explicit examples containing standard model states.

In some sense conformality provides more rigid constraints than supersymmetry. It can predict additional states at $\mathrm{TeV}$ scale, while there can be far fewer initial parameters in conformality models than in SUSY models. Conformality also provides a new approach to gauge coupling unification. It confronts naturalness and provides cancellation of quadratic divergences. The requirements of anomaly cancellationsi can lead to conformality of $\mathrm{U}(1)$ couplings.

There is a viable dark matter candidate, and proton decay can be consistent with experiment.

What is the physical intuition and picture underlying conformality? Consider going to an energy scale higher than the weak scale, for example at the TeV scale. Quark and lepton masses, QCD and weak scales small compared to TeV scale. They may be approximated by zero. The theory is then classically conformally invariant though not at the quantum level because the standard model has non-vanishing renormalization group beta functions and anomalous dimensions. So this suggests that we

add degrees of freedom to yield a gauge field theory with conformal invariance. There will be 't Hooft's naturalness since the zero mass limit increases symmetry to conformal symmetry. 
We have no full understanding of how four-dimensional conformal symmetry can be broken spontaneously so breaking softly by relevant operators is a first step. The theory is assumed to be given by the action:

$$
S=S_{0}+\int d^{4} x \alpha_{i} O_{i}
$$

where $S_{0}$ is the action for the conformal theory and the $O_{i}$ are operators with dimension below four (i.e. relevant) which break conformal invariance softly.

The mass parameters $\alpha_{i}$ have mass dimension $4-\Delta_{i}$ where $\Delta_{i}$ is the dimension of $O_{i}$ at the conformal point.

Let $M$ be the scale set by the parameters $\alpha_{i}$ and hence the scale at which conformal invariance is broken. Then for $E>>M$ the couplings will not run while they start running for $E<M$. To solve the hierarchy problem we assume $M$ is near the $\mathrm{TeV}$ scale. 


\subsection{Experimental evidence for conformality}

Consider embedding the standard model gauge group according to:

$$
S U(3) \times S U(2) \times U(1) \subset \bigotimes_{i} U\left(N d_{i}\right)
$$

Each gauge group of the SM can lie entirely in a $S U\left(N d_{i}\right)$ or in a diagonal subgroup of a number thereof.

Only bifundamentals (including adjoints) are possible. This implies no $(8,2),(3,3)$, etc. A conformality restriction which is new and satisfied in Nature! The fact that the standard model has matter fields all of which can be accommodated as bifundamentals is expermental evidence for conformality.

No $U(1)$ factor can be conformal in perturbation theory and so hypercharge is quantized through its incorporation in a nonabelian gauge group. This is the "conformality" equivalent to the GUT charge quantization condition in e.g. $S U(5)$. It can explain the neutrality of the hydrogen atom. While these are postdictions, the predictions of the theory are new particles, perhaps at a low mass scale, filling out bifundamental representations of the gauge group that restore conformal invariance. The next section will begin our study of known quiver gauge theories from orbifolded $A d S^{5} \times S^{5}$. 


\section{Tabulation of the simplest abelian quivers}

We consider the compactification of the type-IIB superstring on the orbifold $A d S_{5} \times S^{5} / \Gamma$ where $\Gamma$ is an abelian group $\Gamma=Z_{p}$ of order $p$ with elements $\exp (2 \pi i A / p), 0 \leq A \leq(p-1)$.

The resultant quiver gauge theory has $\mathcal{N}$ residual supersymmetries with $\mathcal{N}=2,1,0$ depending on the details of the embedding of $\Gamma$ in the $S U(4)$ group which is the isotropy of the $S^{5}$. This embedding is specified by the four integers $A_{m}, 1 \leq m \leq 4$ with

$$
\Sigma_{m} A_{m}=0 \bmod p
$$

which characterize the transformation of the components of the defining representation of $S U(4)$. We are here interested in the non-supersymmetric case $\mathcal{N}=0$ which occurs if and only if all four $A_{m}$ are non-vanishing.

Table I. All abelian quiver theories with $\mathcal{N}=0$ from $Z_{2}$ to $Z_{7}$. 


\begin{tabular}{||c||c||c|c||c|c|c||c|}
\hline \hline \hline & $\mathrm{p}$ & $A_{m}$ & $a_{i}$ & $\begin{array}{c}\text { scal } \\
\text { bfds }\end{array}$ & $\begin{array}{c}\text { scal } \\
\text { adjs }\end{array}$ & $\begin{array}{c}\text { chir } \\
\text { frms }\end{array}$ & SM \\
\hline \hline 1 & 2 & $(1111)$ & $(000)$ & 0 & 6 & No & No \\
\hline \hline 2 & 3 & $(1122)$ & $(001)$ & 2 & 4 & No & No \\
\hline \hline 3 & 4 & $(2222)$ & $(000)$ & 0 & 6 & No & No \\
4 & 4 & $(1133)$ & $(002)$ & 2 & 4 & No & No \\
5 & 4 & $(1223)$ & $(011)$ & 4 & 2 & No & No \\
6 & 4 & $(1111)$ & $(222)$ & 6 & 0 & Yes & No \\
\hline \hline 7 & 5 & $(1144)$ & $(002)$ & 2 & 4 & No & No \\
8 & 5 & $(2233)$ & $(001)$ & 2 & 4 & No & No \\
9 & 5 & $(1234)$ & $(012)$ & 4 & 2 & No & No \\
10 & 5 & $(1112)$ & $(222)$ & 6 & 0 & Yes & No \\
11 & 5 & $(2224)$ & $(111)$ & 6 & 0 & Yes & No \\
\hline \hline 12 & 6 & $(3333)$ & $(000)$ & 0 & 6 & No & No \\
13 & 6 & $(2244)$ & $(002)$ & 2 & 4 & No & No \\
14 & 6 & $(1155)$ & $(002)$ & 2 & 4 & No & No \\
15 & 6 & $(1245)$ & $(013)$ & 4 & 2 & No & No \\
16 & 6 & $(2334)$ & $(011)$ & 4 & 2 & No & No \\
17 & 6 & $(1113)$ & $(222)$ & 6 & 0 & Yes & No \\
18 & 6 & $(2235)$ & $(112)$ & 6 & 0 & Yes & No \\
19 & 6 & $(1122)$ & $(233)$ & 6 & 0 & Yes & No \\
\hline \hline 20 & 7 & $(1166)$ & $(002)$ & 2 & 4 & No & No \\
21 & 7 & $(3344)$ & $(001)$ & 2 & 4 & No & No \\
22 & 7 & $(1256)$ & $(013)$ & 4 & 2 & No & No \\
23 & 7 & $(1346)$ & $(023)$ & 4 & 2 & No & No \\
24 & 7 & $(1355)$ & $(113)$ & 6 & 0 & No & No \\
25 & 7 & $(1114)$ & $(222)$ & 6 & 0 & Yes & No \\
26 & 7 & $(1222)$ & $(333)$ & 6 & 0 & Yes & No \\
27 & 7 & $(2444)$ & $(111)$ & 6 & 0 & Yes & No \\
28 & 7 & $(1123)$ & $(223)$ & 6 & 0 & Yes & Yes \\
29 & 7 & $(1355)$ & $(113)$ & 6 & 0 & Yes & Yes \\
30 & 7 & $(1445)$ & $(113)$ & 6 & 0 & Yes & Yes \\
\hline \hline \hline
\end{tabular}




\section{Chiral fermions}

The gauge group is $U(N)^{p}$. The fermions are all in the bifundamental representations

$$
\Sigma_{m=1}^{m=4} \Sigma_{j=1}^{j=p}\left(N_{j}, \bar{N}_{j+A_{m}}\right)
$$

which are manifestly non-supersymmetric because no fermions are in adjoint representations of the gauge group. Scalars appear in representations

$$
\Sigma_{i=1}^{i=3} \Sigma_{j=1}^{i=p}\left(N_{j}, \bar{N}_{j \pm a_{i}}\right)
$$

in which the six integers $\left(a_{i},-a_{i}\right)$ characterize the transformation of the antisymmetric second-rank tensor representation of $S U(4)$. The $a_{i}$ are given by $a_{1}=\left(A_{2}+A_{3}\right), a_{2}=\left(A_{3}+A_{1}\right)$, and $a_{3}=\left(A_{1}+A_{2}\right)$.

It is possible for one or more of the $a_{i}$ to vanish in which case the corresponding scalar representation in the summation in Eq.(3) is to be interpreted as an adjoint representation of one particular $U(N)_{j}$. One may therefore have zero, two, four or all six of the scalar representations, in Eq.(33), in such adjoints. One purpose of the present article is to investigate how the renormalization properties and occurrence of quadratic divergences depend on the distribution of scalars into bifundamental and adjoint representations.

Note that there is one model with all scalars in adjoints for each even value of $p$. For general even $p$ the embedding is $A_{m}=$ $\left(\frac{p}{2}, \frac{p}{2}, \frac{p}{2}, \frac{p}{2}\right)$. This series by itself forms the complete list of $\mathcal{N}=0$ abelian quivers with all scalars in adjoints.

To be of more phenomenolgical interest the model should contain chiral fermions. This requires that the embedding be complex: $A_{m} \not \equiv-A_{m}(\bmod \mathrm{p})$. It will now be shown that for the presence of chiral fermions all scalars must be in bifundamentals.

The proof of this assertion follows by assuming the contrary, that there is at least one adjoint arising from, say, $a_{1}=0$. 
Therefore $A_{3}=-A_{2}(\bmod \mathrm{p})$. But then it follows from Eq.(2) that $A_{1}=-A_{4}(\bmod \mathrm{p})$. The fundamental representation of $S U(4)$ is thus real and fermions are non-chiral \#1.

The converse also holds: If all $a_{i} \neq 0$ then there are chiral fermions. This follows since by assumption $A_{1} \neq-A_{2}$, $A_{1} \neq-A_{3}, A_{1} \neq-A_{4}$. Therefore reality of the fundamental representation would require $A_{1} \equiv-A_{1}$ hence, since $A_{1} \neq 0, p$ is even and $A_{1} \equiv \frac{p}{2}$; but then the other $A_{m}$ cannot combine to give only vector-like fermions.

It follows that:

In an $\mathcal{N}=0$ quiver gauge theory, chiral fermions are possible

if and only if all scalars are in bifundamental representaions.

${ }^{\# 1}$ This is almost obvious but for a complete justification, see [173] 


\section{$6 \quad$ Model building}

The next step is to examine how the framework of quiver gauge theories can accommodate, as a sub theory, the standard model. This requires that the standard model gauge group and the three families of quarks and leptons with their correct quantum numbers be accommodated.

In such model building a stringent requirement is that the scalar sector, prescribed by the quiver construction, can by acquiring vacuum expection, values break the symmetry spontaneously to the desired sub theory. This is unlike most other model building where one chooses the representations for the scalars to accomplish this goal. Here the representations for the scalars are dictated by the orbifold construction.

One useful guideline in the symmetry breaking is that to break a semi-simple $S U(N)^{n}$ gauge group to its $S U(N)$ diagonal subgroup requires at least $(n-1)$ bifundamental scalars, connected to one another such that each of the $n S U(N)$ factors is linked to all of the others: it is insufficient if the bifundamental scalars fragment into disconnected subsets.

We shall describe in turn abelian orbifolds [14,174-177] and non abelian orbifolds [178-181] As will become clear abelian orbifolds lead to accommodation of the standard model in unified groups $S U(3)^{n}$ while non abelian orbifolds can lead naturally to incorportion of the standard model in gauge groups such as $S U(4) \times S U(2) \times S U(2)$ and generalizations. 


\subsection{Abelian model building}

We will now classify compactifications of the type IIB superstring on $A d S_{5} \times S^{5} / \Gamma$, where $\Gamma$ is an abelian group of order $n \leq 12$. Appropriate embedding of $\Gamma$ in the isometry of $S^{5}$ yields both $S U S Y$ and non-SUSY chiral models that can contain the minimal $S U S Y$ standard model or the standard model. New non-SUSY three family models with $\Gamma=Z_{8}$ are introduced, which lead to the right Weinberg angle for $\mathrm{TeV}$ trinification.

We find $78 \mathcal{N}=0$ chiral $Z_{n}$ models and discuss a few of phenomenological interest. For $\mathcal{N}=1$ his results in 60 chiral $Z_{n}$ models, and a systematic analysis with $n<8$ yields four containing the minimal SUSY standard model with three families. One of these models extends to an infinite sequence of threefamily $M S S M s$. We also give a lower bound on the number of chiral models for all values of $n$. For completeness, we also discuss abelian models where the orbifolding groups are products of cyclic groups.

\subsubsection{Abelian non-SUSY models}

When one bases models on conformal field theory gotten from the large $N$ expansion of the $A d S / C F T$ correspondence [13], stringy effects can arise at an energy scale as low as a few $\mathrm{TeV}$. These models can potentially test string theory and examples with low energy scales are known in orbifolded $A d S_{5} \times S^{5}$. The first three-family $A d S_{5} \times S^{5} / \Gamma$ model of this type had $\mathcal{N}=1 S U S Y$ and was based on a $\Gamma=Z_{3}$ orbifold [182], see also [183]. However, since then some of the most studied examples have been models without supersymmetry based on both abelian [14,174-177] and non-abelian [178-181] orbifolds of $A d S_{5} \times S^{5}$. Recently both SUSY and nonSUSY three family $Z_{12}$ orbifold models [184-186] have been shown to unify at a low scale $(\sim 4 \mathrm{TeV})$ and to have the promise of testability. One motivation for studying the non-SUSY case is that the 
need for supersymmetry is less clear as: (1) the hierarchy problem is absent or ameliorated, (2) the difficulties involved in breaking the remaining $\mathcal{N}=1 S U S Y$ can be avoided if the orbifolding already results in completely broken $S U S Y$, and (3) many of the effects of $S U S Y$ are still present in the theory, just hidden. For example, the bose-fermi state count matches, RG equations preserve vanishing $\beta$ functions to some number of loops, etc. Here we concentrate on abelian orbifolds with and without supersymmetry, where the orbifolding group $\Gamma$ has order $n=o(\Gamma) \leq 12$. We systematically study those cases with chiral matter (i.e., in the $S U S Y$ case, those with an imbalance between chiral supermultiplets and anti-chiral supermultiplets, and in the non-SUSY case with a net imbalance between left and right handed fermions). We find all chiral models for $n \leq 12$. Several of these contain the standard model $(S M)$ or the minimal supersymmetric standard model $(M S S M)$ with three or four families.

A summary of how orbifolded $A d S_{5} \times S^{5}$ models are constructed was provided in Section 2.

For $\mathcal{N}=0$ the fermions are given by $\sum_{i} \boldsymbol{4} \otimes R_{i}$ and the scalars by $\sum_{i} \mathbf{6} \otimes R_{i}$ where the set $\left\{R_{i}\right\}$ runs over all the irreps of $\Gamma$. For $\Gamma$ abelian, the irreps are all one dimensional and as a consequence of the choice of $N$ in the $1 / N$ expansion, the gauge group [187] is $S U(N)^{n}$. In the $\mathcal{N}=1 \quad S U S Y$ case, chiral supermultiples generated by this embedding are given by $\sum_{i} \mathbf{4} \otimes R_{i}$ where again $\left\{R_{i}\right\}$ runs over all the (irreps) of $\Gamma$. As before, for abelian $\Gamma$, the irreps are all one dimensional and the gauge group is $S U^{n}(N)$. Chiral models require the 4 to be complex $\left(4 \neq 4^{*}\right)$ while a proper embedding requires $6=6^{*}$ where $\mathbf{6}=(\mathbf{4} \otimes \mathbf{4})_{\text {antisym. }}$. (Even though the $\mathbf{6}$ does not enter the model in the $\mathcal{N}=1$ SUSY case, mathematical consistency requires $\mathbf{6}=\mathbf{6}^{*}$, see [173].)

We now have the required background to begin building chiral models. We choose $N=3$ throughout. This means that 
most of our models will proceed to the SM or MSSM through trinification. It is also possible to start with larger $N$, say $N=4$ and proceed to the SM or MSSM via Pati-Salam models. The analysis is similar, so the $N=3$ case is sufficient to gain an understanding of the techniques needed for model building, what choice of $N$ leads to the optimal model is still an open question.

If $S U_{L}(2)$ and $U_{Y}(1)$ are embedded in diagonal subgroups $S U^{p}(3)$ and $S U^{q}(3)$ respectively, of the initial $S U^{n}(3)$, the ratio $\frac{\alpha_{2}}{\alpha_{Y}}$ is $\frac{p}{q}$, leading to a calculable initial value of $\theta_{W}$ with, $\sin ^{2} \theta_{W}=\frac{3}{3+5\left(\frac{p}{q}\right)}$. The more standard approach is to break the initial $S U^{n}(3)$ to $S U_{C}(3) \otimes S U_{L}(3) \otimes S U_{R}(3)$ where $S U_{L}(3)$ and $S U_{R}(3)$ are embedded in diagonal subgroups $S U^{p}(3)$ and $S U^{q}(3)$ of the initial $S U^{n}(3)$. We then embed all of $S U_{L}(2)$ in $S U_{L}(3)$ but $\frac{1}{3}$ of $U_{Y}(1)$ in $S U_{L}(3)$ and the other $\frac{2}{3}$ in $S U_{R}(3)$. This modifies the $\sin ^{2} \theta_{W}$ formula to: $\sin ^{2} \theta_{W}=\frac{3}{3+5\left(\frac{\alpha_{2}}{\alpha_{Y}}\right)}=\frac{3}{3+5\left(\frac{3 p}{p+2 q}\right)}$, which coincides with the previous result when $p=q$. One should use the second (standard) embedding when calculating $\sin ^{2} \theta_{W}$ for any of the models obtained below. A similar relation holds for Pati-Salam type models [180] and their generalizations [188], but this would require investigation of models with $N \geq 4$ which are not included in this review. Note, if $\Gamma=Z_{n}$ the initial $\mathcal{N}=0$ orbifold model (before any symmetry breaking) is completely fixed (recall we always are taking $N=3$ ) by the choice of $n$ and the embedding $\mathbf{4}=\left(\alpha^{i}, \alpha^{j}, \alpha^{k}, \alpha^{l}\right)$, so we define these models by $M_{i j k l}^{n}$. The conjugate models $M_{n-i, n-j, n-k, n-l}^{n}$ contain the same information, so we need not study them separately.

\section{$\mathcal{N}=0$ chiral $Z_{n}$ models}

To get a feel for the constructions, we begin this section by studying the first few $\mathcal{N}=0$ chiral $Z_{n}$ models. Insights gained here will allow us to generalize and give results to arbitrary $n$. First, when $\mathcal{N}=0$, the only allowed $\Gamma=Z_{2}$ orbifold where 
$\mathbf{4}=(\alpha, \alpha, \alpha, \alpha)$ and $Z_{3}$ orbifold where $\mathbf{4}=\left(\alpha, \alpha, \alpha^{2}, \alpha^{2}\right)$ have only real representations and therefore will not yield chiral models. Next, for $\Gamma=Z_{4}$ the choice $\boldsymbol{4}=(\alpha, \alpha, \alpha, \alpha)$ with $N=3$ where $\alpha=e^{\frac{\pi i}{2}}$ (in what follows we will write $\alpha=e^{\frac{2 \pi i}{n}}$ for the roots of unity that generate $Z_{n}$ ), yields an $S U(3)^{4}$ chiral model with the fermion content shown in Table 2. The scalar content of this model is given in Table 3 and a VEV for say a $(3,1, \overline{3}, 1)$ breaks the symmetry to $S U_{D}(3) \times S U_{2}(3) \times S U_{4}(3)$ but renders the model vectorlike, and hence uninteresting, so we consider it no further. (We consider only VEVs that cause symmetry breaking of the type $S U(N) \times S U(N) \rightarrow S U_{D}(N)$. Other symmetry breaking patterns are possible, but for the sake of simplicity they will not be studied here. It is clear from this and previous remarks that there are many phenomenological avenues involving quiver gauge theories yet to be explored.) The only other choice of embedding is a nonpartition model with $\Gamma=Z_{4}$ is $4=\left(\alpha, \alpha, \alpha, \alpha^{3}\right)$ but it leads to the same scalars with half the chiral fermions so we move on to $Z_{5}$.

There is one chiral model for $\Gamma=Z_{5}$ and it is fixed by choosing $\mathbf{4}=\left(\alpha, \alpha, \alpha, \alpha^{2}\right)$, leading to $\mathbf{6}=\left(\alpha^{2}, \alpha^{2}, \alpha^{2}, \alpha^{3}, \alpha^{3}, \alpha^{3}\right)$ with real scalars. It is straightforward to write down the particle content of this $M_{1112}^{5}$ model. The best one can do toward the construction of the standard model is to give a VEV to a $(3,1, \overline{3}, 1,1)$ to break the $S U^{5}(3)$ symmetry to $S U_{D}(3) \times$ $S U_{2}(3) \times S U_{4}(3) \times S U_{5}(3)$. Now a VEV for $(1,3, \overline{3}, 1)$ completes the breaking to $S U^{3}(3)$, but the only remaining chiral fermions are $2[(3, \overline{3}, 1)+(1,3, \overline{3})+(\overline{3}, 1,3)]$ which contains only two families.

Moving on to $\Gamma=Z_{6}$ we find two models where, as with the previous $Z_{5}$ model, the 4 is arranged so that $\mathbf{4}=\left(\alpha^{i}, \alpha^{j}, \alpha^{k}, \alpha^{l}\right)$ with $i+j+k+l=n$. These have $\mathbf{4}=\left(\alpha, \alpha, \alpha, \alpha^{3}\right)$ and $\mathbf{4}=$ $\left(\alpha, \alpha, \alpha^{2}, \alpha^{2}\right)$ and were defined as partition models in [183] when $i$ was equal to zero. Here we generalize and call all models satisfying $i+j+k+l=n$ partition models. We have now 
introduced most of the background and notation we need, so at this point (before completing the investigation of the $\Gamma=Z_{6}$ models) it is useful to give a summary (see Table 4 ) of all $\mathcal{N}=0$ chiral $Z_{n}$ models with real 6's for $n \leq 12$. We note that the $n=8$ partition model with $4=\left(\alpha, \alpha, \alpha^{2}, \alpha^{4}\right)$ has $\chi / N^{2}=16$; the other four have $\chi / N^{2}=32$. Of the nine $Z_{10}$ partition models, 2 have $\chi / N^{2}=30$ and the other 7 have $\chi / N^{2}=40$. The $Z_{12}$ partition models derived from $\mathbf{4}=\left(\alpha, \alpha, \alpha^{4}, \alpha^{6}\right), \mathbf{4}=\left(\alpha, \alpha^{2}, \alpha^{3}, \alpha^{6}\right)$, and $4=\left(\alpha^{2}, \alpha^{2}, \alpha^{2}, \alpha^{6}\right)$ have $\chi / N^{2}=36$; the others have $\chi / N^{2}=48$.

A new class of models appears in Table 4; these are the double partition models. They have $i+j+k+l=2 n$ and none are equivalent to single partition models (if we require that $i, j$, $k$, and $l$ are all positive integers) with $i+j+k+l=n$. The $\mathcal{N}=1$ nonpartition models have been classified [181], and we find eleven $\mathcal{N}=0$ examples in Table 4 . While they have a self conjugate $\mathbf{6}$, this is only a necessary condition that may be insufficient to insure the construction of viable string theory based models [186]. However, as is the $\mathcal{N}=1$ case, the $\mathcal{N}=0$ nonpartition models may still be interesting phenomenologically and as a testing ground for models with the potential of broken conformal invariance.

For $Z_{n}$ orbifold models with $n$ a prime number, only partition models arise. The non-partition and double partition models only occur when $n$ is not a prime number, and only a few are independent. Consider $n=12$, here we can write $Z_{12}=Z_{4} \times Z_{3}$. If we write an element of this group as $\gamma \equiv(a, b)$, where $a$ is a generator of $Z_{4}$ and $b$ of $Z_{3}$, then $\gamma^{2} \equiv\left(a^{2}, b^{2}\right), \gamma^{3} \equiv\left(a^{3}, 1\right)$, etc. The full group is generated by any one of the elements $\gamma=(a, b)$, $\gamma^{5}=\left(a, b^{2}\right), \gamma^{7}=\left(a^{3}, b\right)$, or $\gamma^{11}=\left(a^{3}, b^{2}\right)$. The other choices do not faithfully represent the group. Letting $\alpha=\gamma^{11}$ give a conjugate model, e.g., it transforms $\left(\alpha, \alpha^{6}, \alpha^{8}, \alpha^{9}\right)$ into $\left(\gamma^{11}, \gamma^{6}, \gamma^{4}, \gamma^{3}\right)$, so this pair of double partition models are equivalent, while letting $\alpha=\gamma^{5}$ transforms $\left(\alpha, \alpha^{6}, \alpha^{8}, \alpha^{9}\right)$ into the equivalent model $\left(\gamma^{5}, \gamma^{6}, \gamma^{4}, \gamma^{9}\right)$, and $\alpha=\gamma^{7}$ transforms $\left(\alpha, \alpha^{6}, \alpha^{8}, \alpha^{9}\right)$ into the 
equivalent model $\left(\gamma^{7}, \gamma^{6}, \gamma^{8}, \gamma^{3}\right)$. Hence a systematic use of these operations on the non-partition and double partition models can reduce them to the equivalence classes listed in the tables.

It is easy to prove we always have a proper embedding (i.e., $\mathbf{6}=\mathbf{6}^{*}$ ) for the $\mathbf{4}=\left(\alpha^{i}, \alpha^{j}, \alpha^{k}, \alpha^{l}\right)$ when $i+j+k+l=n$ (or $2 n)$. To show this note from $\mathbf{6}=(\mathbf{4} \otimes \mathbf{4})_{\text {antisym }}$ we find

$$
\mathbf{6}=\left(\alpha^{i+j}, \alpha^{i+k}, \alpha^{i+l}, \alpha^{j+k}, \alpha^{j+l}, \alpha^{k+l}\right)
$$

but $(i+j)=n-k-l=-(k+l)(\bmod n)$ and $(i+k)=$ $n-j-l=-(j+l)(\bmod n)$, so this gives

$$
\mathbf{6}=\left(\alpha^{-(k+l)}, \alpha^{-(j+l)}, \alpha^{-(j+k)}, \alpha^{j+k}, \alpha^{j+l}, \alpha^{k+l}\right)=\mathbf{6}^{*} .
$$

A simple modification of this proof also applies to the double partition models.

Now let us return to $\Gamma=Z_{6}$ where the partition models of interest are: (1) $\mathbf{4}=\left(\alpha, \alpha, \alpha^{2}, \alpha^{2}\right)$ where one easily sees that VEVs for $(3,1, \overline{3}, 1,1,1)$ and then $(1,3, \overline{3}, 1,1)$ lead to at most two families, while other SSB routes lead to equal or less chirality; and (2) $\mathbf{4}=\left(\alpha, \alpha, \alpha, \alpha^{3}\right)$ where VEVs for $(3,1, \overline{3}, 1,1,1)$ followed by a VEV for $(1,3, \overline{3}, 1,1)$ leads to an $S U(3)^{4}$ model containing fermions $2[(3, \overline{3}, 1,1)+(1,3, \overline{3}, 1)+(1,1,3, \overline{3})+(\overline{3}, 1,1,3)]$. However, there are insufficient scalars to complete the symmetry breaking to the standard model. In fact, one cannot even achieve the trinification spectrum.

The double partition $Z_{6}$ model $\mathbf{4}=\left(\alpha, \alpha^{3}, \alpha^{4}, \alpha^{4}\right)$ is relatively complicated, since there are 24 different scalar representations in the spectrum, and this makes the SSB analysis rather difficult. A number of possible SSB pathways were investigated [190], but none were found that lead to the SM with at least three families. However, since the search was not exhaustive, we cannot make a definitive statement about this model. As stated elsewhere, the non-partition models are difficult to interpret, if not patho- 
logical, so we have not studied the SSB pathways for these $Z_{6}$ models.

We move on to $Z_{7}$, where there are three partition models: (1) for $\mathbf{4}=\left(\alpha, \alpha^{2}, \alpha^{2}, \alpha^{2}\right)$, we find no SSB pathway to the SM. There are paths to an SM with less than three families, e. g., VEVs for $(3,1,1, \overline{3}, 1,1,1),(1,3,1, \overline{3}, 1,1),(3, \overline{3}, 1,1,1)$, and $(1,3, \overline{3}, 1)$ lead to one family at the $S U^{3}(3)$ level; (2) for $\mathbf{4}=\left(\alpha, \alpha, \alpha, \alpha^{4}\right)$, again we find only paths to family-deficient standard models. An example is where we have VEVs for $(3,1, \overline{3}, 1,1,1,1),(1,3, \overline{3}, 1,1,1)$, $(3,1, \overline{3}, 1,1)$, and $(1,3, \overline{3}, 1)$, which lead to a two-family $S U^{3}(3)$ model; (3) finally, $\mathbf{4}=\left(\alpha, \alpha, \alpha^{2}, \alpha^{3}\right)$ is the model discovered in [176], where VEVs to $(1,3,1, \overline{3}, 1,1,1),(1,1,3, \overline{3}, 1,1),(1,1,3, \overline{3}, 1)$ and $(1,1,3, \overline{3})$ lead to a three family model with the correct Weinberg angle at the $Z$-pole, $\sin ^{2} \theta_{W}=3 / 13$.

For $Z_{n}$ with $n \geq 8$, the number of representations of matter multiplets has already grown to a degree where it makes a systematic analysis of the models prohibitively time-consuming. It is thus helpful to have further motivation to study particular examples or limited sets of these models with large $n$ values. Thus we searched for examples which break $S U(3)^{8}$ down to diagonal subgroups $S U(3)^{4} \times S U(3)^{3} \times S U(3)$, since this implies the right Weinberg angle for $\mathrm{TeV}$ trinification [163], $\sin ^{2} \theta_{W}=3 / 13$, when embedding $S U(3)_{L}$ and $S U(3)_{R}$ into the diagonal subgroups of $S U(3)^{4}$ and $S U(3)$, respectively. There are actually 11 different possibilities to break $S U(3)^{8}$ down to $S U(3)^{4} \times S U(3)^{3} \times S U(3)$, assuming the necessary scalars exist. While none of these paths was successful for $\mathbf{4}=\left(\alpha, \alpha, \alpha, \alpha^{5}\right)$, the model $\mathbf{4}=\left(\alpha, \alpha, \alpha^{2}, \alpha^{4}\right)$ leads to the 3 family SM. Assigning VEVs to $(3,1, \overline{3}, 1,1,1,1,1)$, $(3,1,1, \overline{3}, 1,1,1),(3, \overline{3}, 1,1,1,1),(1,3, \overline{3}, 1,1)$ and $(1,3, \overline{3}, 1)$ breaks $S U(3)^{8}$ down to $S U(3)_{1235} \times S U(3)_{467} \times S U(3)_{8}$.

Another option exists for $\mathbf{4}=\left(\alpha, \alpha^{4}, \alpha^{5}, \alpha^{6}\right)$, when assigning VEVs to \#2

\footnotetext{
\#2 This SSB pathway has first been derived by Yasmin Anstruthler.
} 


$$
\begin{aligned}
& (3, \overline{3}, 1,1,1,1,1,1),(3, \overline{3}, 1,1,1,1,1),(3,1,1, \overline{3}, 1,1), \\
& (1,3, \overline{3}, 1,1) \text { and }(1,3,1, \overline{3})
\end{aligned}
$$

These models have not been discussed in the literature so far and have potential interesting phenomenology.

\subsection{2 $\mathcal{N}=1$ chiral $Z_{n}$ models}

To tabulate the possible models for each value of $n$, we first show that a proper embedding (i.e., $\left.\mathbf{6}=\mathbf{6}^{*}\right)$ for $\mathbf{4}=\left(\mathbf{1}, \alpha^{i}, \alpha^{j}, \alpha^{k}\right)$ results when $i+j+k=n$. To do this we use the fact that the conjugate model has $i \rightarrow i^{\prime}=n-i, j \rightarrow j^{\prime}=n-j$ and $k \rightarrow k^{\prime}=n-k$. Summing we find $i^{\prime}+j^{\prime}+k^{\prime}=3 n-(i+j+k)=2 n$. But from $\mathbf{6}=(\mathbf{4} \otimes \mathbf{4})_{\text {antisym }}$ we find $\mathbf{6}=\left(\alpha^{i}, \alpha^{j}, \alpha^{k}, \alpha^{j+k}, \alpha^{i+k}, \alpha^{i+j}\right)$, but $i+j=n-k=k^{\prime}$. Likewise $i+k=j^{\prime}$ and $j+k=i^{\prime}$ so $\mathbf{6}=\left(\alpha^{i}, \alpha^{j}, \alpha^{k}, \alpha^{i^{\prime}}, \alpha^{j^{\prime}}, \alpha^{k^{\prime}}\right)$ and this is $\mathbf{6}^{*}$ up to an automorphism which is sufficient to provide a proper embedding (or to provide real scalars in the non-SUSY models). Models with $i+j+k=n$ (we will call these partition models) are always chiral, with total chirality (number of chiral states) $\chi=3 N^{2} n$ except in the case where $n$ is even and one of $i, j$, or $k$ is $n / 2$ where $\chi=2 N^{2} n$. (No more than one of $i, j$, and $k$ can be $n / 2$ since they sum to $n$ and are all positive.) This immediately gives us a lower bound on the number of chiral models at fixed $n$. It is the number of partitions of $n$ into three non-negative integers. There is another class of models with $i^{\prime}=k$ and $j^{\prime}=2 j$, and total chirality $\chi=N^{2} n$; for example a $Z_{9}$ orbifold with $\mathbf{4}=\left(\mathbf{1}, \alpha^{3}, \alpha^{3}, \alpha^{6}\right)$. And there are a few other sporadically occurring cases like $M_{124}^{6}$, which typically have reduced total chirality, $\chi<3 N^{2} n$. Such "nonpartition" - i.e. neither partition nor double partition - models can fail other more subtle constraints on consistent embedding [173], but we list them here because they have vanishing anomaly coefficients and vanishing one loop $\beta$ functions, and so are still of 
phenomenological interest from the gauge theory model building perspective.

We now list all the $\mathcal{N}=1, Z_{n}$ orbifold models up to $n=12$ along with the total chirality of each model, (see Table 1 ).

A systematic search through $n \leq 7$ yields four models that can result a in three-family MSSM. They are $M_{111}^{3}, M_{122}^{5}, M_{123}^{6}$, and $M_{133}^{7}$. There may be many more models with sensible phenomenology at larger $n$, and we have given one example $M_{333}^{9}$, with particularly simple spontaneous symmetry breaking, that is also a member of an infinite series of models $M_{\frac{n}{3} \frac{n}{3} \frac{n}{3}}^{n}$, which all can lead to three-family MSSMs. The value of $\sin ^{2} \theta_{W}$ at $S U^{n}(3)$ unification was calculated for all these three family models in [183]. This completes the summary of $\mathcal{N}=1$ chiral $Z_{n}$ models, so we now proceed to investigate chiral $Z_{n}$ models with no remaining supersymmetry.

\subsection{3 $\mathcal{N}=0,1$ chiral models for abelian product groups}

Now let us consider abelian orbifold groups of order $g \leq 12$, that are not just $Z_{n}$. There are only four, but they will be sufficient to teach us how to deal with this type of orbifold. We will search for both $\mathcal{N}=1$ and $\mathcal{N}=0$ models. Three groups, $Z_{2} \times Z_{4}, Z_{3} \times Z_{3}$, and $Z_{2} \times Z_{2} \times Z_{3}$ fit our requirements. We have dispensed with $Z_{2} \times Z_{2} \times Z_{2}$ since all its fermionic representations are real and it cannot produce chiral models.

First for $Z_{2} \times Z_{4}$, we can write elements as $\left(\alpha^{i}, \beta^{i^{\prime}}\right)$ where $\alpha^{2}=$ 1 , and $\beta^{4}=1$. As usual, the supersymmetry after orbifolding is determined by the embeddings. These are of the form:

$$
\mathbf{4}=\left(\left(\alpha^{i}, \beta^{i^{\prime}}\right),\left(\alpha^{j}, \beta^{j^{\prime}}\right),\left(\alpha^{k}, \beta^{k^{\prime}}\right),\left(\alpha^{l}, \beta^{l^{\prime}}\right)\right) .
$$

If all four entries are nontrivial $\mathcal{N}=0 S U S Y$ results, if one is trivial, then we have $\mathcal{N}=1$. We can think of the $S U S Y$ breaking as a two step process, where we first embed the $\alpha$ 's 
in the 4 and then the $\beta$ 's. Let us proceed this way and include only the partition, and possibly double partition models. (As we noted above, the nonpartition models have potential pathologies.) Thus for the $\alpha$ 's we must have either $\mathbf{4}_{\alpha_{1}}=(-1,-1,-1,-1)$ or $\mathbf{4}_{\alpha_{2}}=(1,1,-1,-1)$. The $\mathbf{4}_{\alpha_{1}}$ results in $\mathcal{N}=0 S U S Y$, while $\boldsymbol{4}_{\alpha_{2}}$ gives $\mathcal{N}=2$. We do not include trivial $Z_{n}$ factors $\mathbf{4}=(1,1,1,1)$ in the discussion, since these models contain very little new information. [Note, for any product groups $Z_{n} \times Z_{m}$, the $\alpha$ 's of $Z_{n}$ must be self conjugate in the $\mathbf{6}$, as are the $\beta$ 's of $Z_{m}$. Hence, the full $\mathbf{6}$ is self conjugate since the subgroups $Z_{n}$ and $Z_{m}$ are orthogonal. This generalizes to more complicated products $Z_{n} \times Z_{m} \times Z_{p} \times \ldots$.

Now for the $\beta^{\prime} s$. These are to be combined with the $\alpha$ 's, so we must consider the $\boldsymbol{4}_{\alpha_{1}}$ and $\boldsymbol{4}_{\alpha_{2}}$ separately. For $\boldsymbol{4}_{\alpha_{1}}$, the inequivalent $\mathbf{4}_{\beta}$ 's are $\mathbf{4}_{\beta_{1}}=(\beta, \beta, \beta, \beta)$ and $\boldsymbol{4}_{\beta_{2}}=\left(1, \beta, \beta, \beta^{2}\right)$. [Models with $\boldsymbol{4}=\left(1,1, \beta^{2}, \beta^{2}\right)$ are uninteresting since they all are nonchiral.] Both cases have $\mathcal{N}=0 S U S Y$ since we were already at $\mathcal{N}=0$ after the $\boldsymbol{4}_{\alpha_{1}}$ embedding. For $\boldsymbol{4}_{\alpha_{2}}$ we find five possible inequivalent embeddings, again we can have $\boldsymbol{4}_{\beta_{\mathbf{1}}}=(\beta, \beta, \beta, \beta)$ or $\mathbf{4}_{\beta_{2}}=\left(1, \beta, \beta, \beta^{2}\right)$, but now we can also have $\boldsymbol{4}_{\beta_{3}}=\left(1, \beta^{2}, \beta, \beta\right)$, $\mathbf{4}_{\beta_{4}}=\left(\beta, \beta, 1, \beta^{2}\right)$ and $\mathbf{4}_{\beta_{5}}=\left(\beta^{2}, \beta, 1, \beta\right)$. The embeddings $\mathbf{4}_{\beta_{\mathbf{1}}}$, $\mathbf{4}_{\beta_{4}}$ and $\boldsymbol{4}_{\beta_{5}}$ lead to $\mathcal{N}=0 S U S Y$ while $\boldsymbol{4}_{\beta_{2}}$ and $\boldsymbol{4}_{\beta_{3}}$ leave $\mathcal{N}=$ 1 SUSY unbroken. A similar analysis can be carried out for $Z_{3} \times Z_{3}$, and $Z_{2} \times Z_{2} \times Z_{3}$, with the obvious generalization to a triple embedding for $Z_{2} \times Z_{2} \times Z_{3}$.

For $Z_{3} \times Z_{3}$ there are five models. We can choose $\mathbf{4}_{\alpha}=$ $(1, \alpha, \alpha, \alpha)$ as the embedding of the first $Z_{3}$. Then the embedding of the second $Z_{3}$ can be $\boldsymbol{4}_{\beta_{1}}=(1, \beta, \beta, \beta), \mathbf{4}_{\beta_{2}}=(\beta, 1, \beta, \beta)$, $\mathbf{4}_{\beta_{\mathbf{3}}}=\left(1,1, \beta, \beta^{2}\right), \mathbf{4}_{\beta_{\mathbf{4}}}=\left(\beta, 1,1, \beta^{2}\right)$, or $\mathbf{4}_{\beta_{\mathbf{5}}}=\left(\beta^{2}, 1,1, \beta\right)$. The first and third result in $\mathcal{N}=1 S U S Y$ models while the other three are $\mathcal{N}=0$.

For $Z_{2} \times Z_{2} \times Z_{3}$ we find 9 chiral models. Rather than belabor the details, we summarize all our results for $Z_{2} \times Z_{4}, Z_{3} \times Z_{3}$, and $Z_{2} \times Z_{2} \times Z_{3}$ in Table 5 . 


\section{Summary}

We have now completed our task of summarizing all $\mathcal{N}=0$ and $\mathcal{N}=1 S U S Y$ chiral models of phenomenological interest derivable from orbifolding $A d S_{5} \times S^{5}$ with abelian orbifold group $\Gamma$ of order $o(\Gamma) \leq 12$. The models fall into three classes: partition models, double partition models, and non-partition models as determined by how the equation $i+j+k+l=s n$ is satisfied by the embedding where $s=1$ for partition models, $s=2$ for double partition models and $s$ is non-integer for non-partition models. For $Z_{n}$ orbifolds with $\mathcal{N}=1 S U S Y$, there are 53 partition models, and 7 non-partition models, and for $\mathcal{N}=0 S U S Y$, we find 54 partition, 11 double partition, and 13 non-partition models. The non-partition models have potential pathologies if they are to be interpreted as coming from string theory, but they still may be of phenomenological and technical interest, so they have been included in our classification of $Z_{n}$ models. See also the related discussions in [140] and [191].

The non $-Z_{n}$ abelian product groups of interest (we only consider partition models here) with $g \leq 12$ are $Z_{2} \times Z_{4}$ with five $\mathcal{N}=0$ and two $\mathcal{N}=1$ chiral models; $Z_{3} \times Z_{3}$ with three $\mathcal{N}=0$ and two $\mathcal{N}=1$ chiral models, and $Z_{2} \times Z_{2} \times Z_{3}$ with seven $\mathcal{N}=0$ and two $\mathcal{N}=1$ chiral models.

The relation to the SM and MSSM have been explored in some detail for $Z_{n}$ models with $g \leq 7$, but we have only given a few examples with $g>7$, and have indicated how to build abelian orbifold models for any $g$. Two $Z_{8}$ models have been introduced, which can lead to the right Weinberg angle, when broken down to the SM. These results should be useful to model builders and phenomenologists alike. 


\begin{tabular}{|c||c|c|c|c|}
\hline$M_{1111}^{4}(F)$ & 1 & $\alpha$ & $\alpha^{2}$ & $\alpha^{3}$ \\
\hline \hline 1 & & $\times^{4}$ & & \\
\hline$\alpha$ & & & $\times^{4}$ & \\
\hline$\alpha^{2}$ & & & & $\times^{4}$ \\
\hline$\alpha^{3}$ & $\times^{4}$ & & & \\
\hline
\end{tabular}

Table 2: Fermion content for the model $M_{1111}^{4}$. The $\times^{4}$ entry at the $(1, \alpha)$ position means the model contains $4(3, \overline{3}, 1,1)$ of $S U^{4}(3)$, etc. Hence, the fermions in this table are $4[(3, \overline{3}, 1,1)+$ $(1,3, \overline{3}, 1)+(1,1,3, \overline{3})+(\overline{3}, 1,1,3)]$. Diagonal entries do not occur in this model but, if they did, an $\times$ at say $\left(\alpha^{2}, \alpha^{2}\right)$ would correspond to $(1,8+1,1,1)$, etc. See models below.

\begin{tabular}{|c||c|c|c|c|}
\hline$M_{1111}^{4}(S)$ & 1 & $\alpha$ & $\alpha^{2}$ & $\alpha^{3}$ \\
\hline \hline 1 & & & $\times^{6}$ & \\
\hline$\alpha$ & & & & $\times^{6}$ \\
\hline$\alpha^{2}$ & $\times^{6}$ & & & \\
\hline$\alpha^{3}$ & & $\times^{6}$ & & \\
\hline
\end{tabular}

Table 3: Scalar content of the model $M_{1111}^{4}$. 


\begin{tabular}{|c|c|c|c|}
\hline$n$ & 4 & $\chi / N^{2}$ & comment \\
\hline "4 & $(\alpha, \alpha, \alpha, \alpha)$ & 16 & $\bar{c} i+j+k+l=3 ;$ one model $(i=j=k=l=1)$ \\
\hline 4 & $\left(\alpha, \alpha, \alpha, \alpha^{3}\right)^{*}$ & 8 & nonpartition model \\
\hline 5 & $\left(\alpha^{i}, \alpha^{j}, \alpha^{k}, \alpha^{l}\right)$ & 20 & $i+j+k+l=5 ; 1$ models \\
\hline 6 & $\left(\alpha^{i}, \alpha^{j}, \alpha^{k}, \alpha^{l}\right)$ & $\leq 24$ & $i+j+k+l=6 ; 2$ models \\
\hline 6 & $\left(\alpha, \alpha, \alpha^{3}, \alpha^{5}\right)^{*}$ & 6 & nonpartition \\
\hline 6 & $\left(\alpha, \alpha^{2}, \alpha^{3}, \alpha^{5}\right)^{*}$ & 6 & nonpartition \\
\hline 6 & $\left(\alpha, \alpha^{3}, \alpha^{4}, \alpha^{4}\right)$ & 24 & double partition \\
\hline 7 & $\left(\alpha^{i}, \alpha^{j}, \alpha^{k}, \alpha^{l}\right)$ & 28 & $i+j+k+l=7 ; 3$ models \\
\hline 8 & $\left(\alpha^{i}, \alpha^{j}, \alpha^{k}, \alpha^{l}\right)$ & $\leq 32$ & $i+j+k+l=8 ; 5$ models \\
\hline 8 & $\left(\alpha, \alpha^{2}, \alpha^{3}, \alpha^{6}\right)^{*}$ & 16 & nonpartition \\
\hline 8 & $\left(\alpha^{2}, \alpha^{2}, \alpha^{2}, \alpha^{6}\right)^{*}$ & 16 & analog of $Z_{4}\left(\alpha, \alpha, \alpha, \alpha^{3}\right)$ model \\
\hline 8 & $\left(\alpha, \alpha^{4}, \alpha^{5}, \alpha^{6}\right)$ & 32 & double partition \\
\hline 9 & $\left(\alpha^{i}, \alpha^{j}, \alpha^{k}, \alpha^{l}\right)$ & 36 & $i+j+k+l=9 ; 7$ models \\
\hline 9 & $\left(\alpha, \alpha^{3}, \alpha^{4}, \alpha^{7}\right)^{*}$ & 36 & nonpartition \\
\hline 9 & $\left(\alpha, \alpha^{4}, \alpha^{6}, \alpha^{7}\right)$ & 36 & double partition \\
\hline 10 & $\left(\alpha^{i}, \alpha^{j}, \alpha^{k}, \alpha^{l}\right)$ & $\leq 40$ & $i+j+k+l=10 ; 9$ models \\
\hline 10 & $\left(\alpha, \alpha^{3}, \alpha^{8}, \alpha^{8}\right)$ & 40 & double partition \\
\hline 10 & $\left(\alpha, \alpha^{5}, \alpha^{6}, \alpha^{8}\right)$ & 40 & double partition \\
\hline 11 & $\left(\alpha^{i}, \alpha^{j}, \alpha^{k}, \alpha^{l}\right)$ & 44 & $i+j+k+l=11 ; 11$ models \\
\hline 12 & $\left(\alpha^{i}, \alpha^{j}, \alpha^{k}, \alpha^{l}\right)$ & $\leq 48$ & $i+j+k+l=12 ; 15$ models \\
\hline 12 & $\left(\alpha, \alpha^{4}, \alpha^{9}, \alpha^{10}\right)$ & 48 & double partition \\
\hline 12 & $\left(\alpha, \alpha^{5}, \alpha^{9}, \alpha^{9}\right)$ & 48 & double partition \\
\hline 12 & $\left(\alpha, \alpha^{6}, \alpha^{7}, \alpha^{10}\right)$ & 48 & double partition \\
\hline 12 & $\left(\alpha, \alpha^{6}, \alpha^{8}, \alpha^{9}\right)$ & 36 & double partition \\
\hline 12 & $\left(\alpha, \alpha^{7}, \alpha^{8}, \alpha^{8}\right)$ & 48 & double partition \\
\hline 12 & $\left(\alpha^{2}, \alpha^{6}, \alpha^{8}, \alpha^{8}\right)$ & 36 & double partition \\
\hline 12 & $\left(\alpha, \alpha, \alpha^{5}, \alpha^{9}\right)^{*}$ & 48 & nonpartition \\
\hline 12 & $\left(\alpha, \alpha^{3}, \alpha^{5}, \alpha^{9}\right)^{*}$ & 24 & nonpartition \\
\hline 12 & $\left(\alpha, \alpha^{3}, \alpha^{7}, \alpha^{11}\right)^{*}$ & 24 & nonpartition \\
\hline 12 & $\left(\alpha, \alpha^{5}, \alpha^{5}, \alpha^{9}\right)^{*}$ & 48 & nonpartition \\
\hline 12 & $\left(\alpha^{2}, \alpha^{2}, \alpha^{6}, \alpha^{10}\right)^{*}$ & 12 & nonpartition \\
\hline 12 & $\left(\alpha^{2}, \alpha^{3}, \alpha^{4}, \alpha^{9}\right)^{*}$ & 24 & nonpartition \\
\hline 12 & $\left(\alpha^{2}, \alpha^{4}, \alpha^{6}, \alpha^{10}\right)^{*}$ & 24 & nonpartition \\
\hline 12 & $\left(\alpha^{3}, \alpha^{3}, \alpha^{3}, \alpha^{9}\right)^{*}$ & 24 & nonpartition \\
\hline
\end{tabular}

Table 4. All chiral $\mathcal{N}=0, Z_{n}$ orbifold models with $n \leq 12$. The 13 non-partition models are marked with an asterisk $\left(^{*}\right)$. For further explanations see text. 


\begin{tabular}{|c||c|c|c|}
\hline Group & $\mathbf{4}$ & $\chi / N^{2}$ & $\mathcal{N}$ \\
\hline \hline$Z_{2} \times Z_{4}$ & $(-1,-1,-1,-1) \times(\beta, \beta, \beta, \beta)$ & 32 & 0 \\
\hline$Z_{2} \times Z_{4}$ & $(-1,-1,-1,-1) \times\left(\mathbf{1}, \beta, \beta, \beta^{2}\right)$ & 16 & 0 \\
\hline$Z_{2} \times Z_{4}$ & $(1,1,-1,-1) \times(\beta, \beta, \beta, \beta)$ & 32 & 0 \\
\hline$Z_{2} \times Z_{4}$ & $(1,1,-1,-1) \times\left(\mathbf{1}, \beta, \beta, \beta^{2}\right)$ & 16 & 1 \\
\hline$Z_{2} \times Z_{4}$ & $(1,1,-1,-1) \times\left(\mathbf{1}, \beta^{2}, \beta, \beta\right)$ & 16 & 1 \\
\hline$Z_{2} \times Z_{4}$ & $(1,1,-1,-1) \times\left(\beta, \beta, 1, \beta^{2}\right)$ & 16 & 0 \\
\hline$Z_{2} \times Z_{4}$ & $(1,1,-1,-1) \times\left(\beta, \beta^{2}, 1, \beta\right)$ & 16 & 0 \\
\hline$Z_{3} \times Z_{3}$ & $(\mathbf{1}, \alpha, \alpha, \alpha) \times(1, \beta, \beta, \beta)$ & 27 & 1 \\
\hline$Z_{3} \times Z_{3}$ & $(\mathbf{1}, \alpha, \alpha, \alpha, \alpha) \times(\beta, 1, \beta, \beta)$ & 36 & 0 \\
\hline$Z_{3} \times Z_{3}$ & $(\mathbf{1}, \alpha, \alpha, \alpha) \times\left(\beta, 1, \beta, \beta^{2}\right)$ & 18 & 1 \\
\hline$Z_{3} \times Z_{3}$ & $(\mathbf{1}, \alpha, \alpha, \alpha) \times\left(\beta^{2}, 1,1, \beta\right)$ & 36 & 0 \\
\hline$Z_{3} \times Z_{3}$ & $(1,1,-1,-1) \times(1,1,-1,-1) \times(1, \gamma, \gamma, \gamma)$ & 36 & 0 \\
\hline$Z_{2} \times Z_{2} \times Z_{3}$ & $(1,1,-1,-1) \times(-1,1,1,-1) \times(1, \gamma, \gamma, \gamma)$ & 48 & 1 \\
\hline$Z_{2} \times Z_{2} \times Z_{3}$ & $(1,1,-1,-1) \times(-1,-1,-1,-1) \times(1, \gamma, \gamma, \gamma)$ & 48 & 0 \\
\hline$Z_{2} \times Z_{2} \times Z_{3}$ & $(-1,-1,1,1) \times(-1,-1,1,1) \times(1, \gamma, \gamma, \gamma)$ & 48 & 0 \\
\hline$Z_{2} \times Z_{2} \times Z_{3}$ & $(-1,-1,1,1) \times(-1,-1,-1,-1) \times(1, \gamma, \gamma, \gamma)$ & 48 & 0 \\
\hline$Z_{2} \times Z_{2} \times Z_{3}$ & $(1,1,-1,-1) \times(-1,-1,1,1) \times(1, \gamma, \gamma, \gamma)$ & 48 & 0 \\
\hline$Z_{2} \times Z_{2} \times Z_{3}$ & $(-1,-1,-1) \times(1,-1,-1,1) \times(1, \gamma, \gamma, \gamma)$ & 48 & 1 \\
\hline$Z_{2} \times Z_{2} \times Z_{3}$ & $(-1,1,-1,1,1) \times(1, \gamma, \gamma, \gamma)$ & 48 & 0 \\
\hline$Z_{2} \times Z_{2} \times Z_{3}$ & $(-1,-1,-1,-1) \times(-1,-1,-1,-1) \times(1, \gamma, \gamma, \gamma)$ & 48 & 0 \\
\hline$Z_{2} \times Z_{2} \times Z_{3}$ & & & \\
\hline
\end{tabular}

Table 5.: All chiral $\mathcal{N}=0$ and $\mathcal{N}=1 S U S Y$ partition models for product orbifolding groups $Z_{2} \times Z_{4}, Z_{3} \times Z_{3}$, and $Z_{2} \times Z_{2} \times$ $Z_{3}$, where the embedding is nontrivial in all factors. Our notation is: $\mathbf{4}=\left(\left(\alpha^{i}\right),\left(\alpha^{j}\right),\left(\alpha^{k}\right),\left(\alpha^{l}\right)\right) \times\left(\left(\beta^{i^{\prime}}\right),\left(\beta^{j^{\prime}}\right),\left(\beta^{k^{\prime}}\right),\left(\beta^{l^{\prime}}\right)\right)=$ $\left(\left(\alpha^{i}, \beta^{i^{\prime}}\right),\left(\alpha^{j}, \beta^{j^{\prime}}\right),\left(\alpha^{k}, \beta^{k^{\prime}}\right),\left(\alpha^{l}, \beta^{l^{\prime}}\right)\right)$, etc. 


\subsubsection{Abelian SUSY models}

Orbifolded $A d S_{5} \times S^{5}$ is fertile ground for building models which can potentially test string theory. When one bases the models on the conformal field theory gotten from the large $N$ expansion of the $A d S / C F T$ correspondence [13], stringy effects can show up at the scale of a few $T e V$. The first three-family model of this type had $\mathcal{N}=1 S U S Y$ and was based on a $Z_{3}$ orbifold [192]. However, since then the most studied examples have been models without supersymmetry based on both abelian [14,174-177] and non-abelian [178-181] orbifolds of $A d S_{5} \times S^{5}$. Here we return to $Z_{n}$ orbifolds with supersymmetry, and systematically study those cases with chiral matter (i.e., those with an imbalance between chiral supermultiplets and anti-chiral supermultiplets). We classify all cases up to $n \leq 12$, and show that several of these contain the minimal supersymmetric standard model (MSSM) with three families.

For details of the construcion of quiver gauge theories from orbifolds, see Section 2.

The replacement of $S^{5}$ by $S^{5} / \Gamma$ reduces the supersymmetry to $\mathcal{N}=0,1$ or 2 from the initial $\mathcal{N}=4$, depending on how $\Gamma$ is embedded in the $S O(6) \sim S U(4)$ isometry of $S^{5}$. The case of interest here is $\mathcal{N}=1 S U S Y$ where $\Gamma$ completely embeds in an $S U(3)$ subgroup of the $S U(4)$ isometry. I.e., we embed $\operatorname{rep}(\Gamma) \rightarrow \mathbf{4}$ of $S U(4)$ as $\mathbf{4}=(\mathbf{1}, \mathbf{r})$ where $\mathbf{1}$ is the trivial irrep of $\Gamma$ and $\mathbf{r}$ is a nontrivial (but possibly reducible) three dimensional representation of $\Gamma$. The chiral supermultiples generated by this embedding are given by

$$
\sum_{i} 4 \otimes R_{i}
$$

where the set $R_{i}$ runs over all the irreps of $\Gamma$. For our choice, $\Gamma=Z_{n}$, the irreps are all one dimensional and as a consequence of the choice of $N$ in the $1 / N$ expansion, the gauge group [187] 
is $U(N)^{n}$. Chiral models require the $\mathbf{4}$ to be complex $(\mathbf{4} \neq$ $\left.4^{*}\right)$ while a proper embedding requires $6=6^{*}$ where $6=(4 \otimes$ 4) antisym., (even though the 6 does not enter the model). This information is sufficient for us to begin our investigation. We will choose $N=3$ throughout, and if we use the fact that $S U_{L}(2)$ and $U_{Y}(1)$ are embedded in diagonal subgroups $S U^{p}(3)$ and $S U^{q}(3)$ of the initial $S U^{n}(3)$, the ratio $\frac{\alpha_{2}}{\alpha_{Y}}$ turns out to be $\frac{p}{q}$. This implies the initial value of $\theta_{W}$ is calculable in these models and $\sin ^{2} \theta_{W}$ satisfies

$$
\sin ^{2} \theta_{W}=\frac{3}{3+5\left(\frac{p}{q}\right)} .
$$

On the other hand, a more standard approach is to break the initial $S U^{n}(3)$ to $S U_{C}(3) \otimes S U_{L}(3) \otimes S U_{R}(3)$ where $S U_{L}(3)$ and $S U_{R}(3)$ are embedded in diagonal subgroups $S U^{p}(3)$ and $S U^{q}(3)$ of the initial $S U^{n}(3)$. We then embed all of $S U_{L}(2)$ in $S U_{L}(3)$ but $\frac{1}{3}$ of $U_{Y}(1)$ in $S U_{L}(3)$ and the other $\frac{2}{3}$ in $S U_{R}(3)$. This modifies the $\sin ^{2} \theta_{W}$ formula to:

$$
\sin ^{2} \theta_{W}=\frac{3}{3+5\left(\frac{\alpha_{2}}{\alpha_{Y}}\right)}=\frac{3}{3+5\left(\frac{3 p}{p+2 q}\right)}
$$

Note, this coincides with the previous result when $p=q$. We will use the later result when calculating $\sin ^{2} \theta_{W}$ below. A similar relation holds for Pati-Salam type models [181].

We now go through a systematic list of low $n$ examples as we did in the $\mathcal{N}=1$ case to familiarize ourselves with $\mathcal{N}=0 \bmod$ els. First a $Z_{2}$ orbifold has only real representations and therefore will not yield a chiral model. (Note, although all matter is in chiral supermultiplet, if there is a left-handed supermultiplet to match each right-handed supermultiplet, then the model has no overall chirality, i.e., it is vectorlike.)

Next, for $\Gamma=Z_{3}$ the choice $\mathbf{4}=(\mathbf{1}, \alpha, \alpha, \alpha)$ with $N=3$ where $\alpha=e^{\frac{2 \pi i}{3}}$ (in what follows we will write $\alpha=e^{\frac{2 \pi i}{n}}$ for $Z_{n}$ ), yields the three family trinification [163] model of [192], but without sufficient scalars to break the gauge symmetry to the 
MSSM. Here the initial value of $\sin ^{2} \theta_{W}=\frac{3}{8}$, so unification at the $\mathrm{TeV}$ scale is also problematic. There is another chiral model for $\mathbf{4}=\left(\mathbf{1}, \alpha, \alpha, \alpha^{2}\right)$ but it can have at most one chiral family.

$Z_{4}$ orbifolds allow only one chiral model with $\mathcal{N}=1 S U S Y$. It is generated by $\mathbf{4}=\left(\mathbf{1}, \alpha, \alpha, \alpha^{2}\right)$ but can have at most two chiral families.

There are two chiral models for $Z_{5}$, and they are fixed by choosing $\mathbf{4}=\left(\mathbf{1}, \alpha, \alpha, \alpha^{3}\right)$ and $\mathbf{4}=\left(\mathbf{1}, \alpha, \alpha^{2}, \alpha^{2}\right)$. Before looking at these in detail, let us pause to define a useful notation for classifying models. The initial model (before any symmetry breaking) is completely fixed (recall we always are taking $N=3$ ) by the choice of $Z_{n}$ and the embedding $\mathbf{4}=\left(\mathbf{1}, \alpha^{i}, \alpha^{j}, \alpha^{k}\right)$, so we define the model to by $M_{i j k}^{n}$. We immediately observe that the conjugate model $M_{n-i, n-j, n-k}^{n}$ contains the same information, so we need not study it separately.

Returning now to $Z_{5}$, the two models are $M_{113}^{5}$ and $M_{122}^{5}$. (Other inconsistent models are eliminated by requiring $\mathbf{6}=\mathbf{6}^{*}$ keeping the number of models limited.) We find no pattern of spontaneous symmetry breaking $(S S B)$ for $M_{113}^{5}$ that yields the $M S S M$, but $M_{122}^{5}$ is more interesting. The matter content of $M_{122}^{5}$ is shown in Table 1.

For each entry, $(x)$, in the table, we have a chiral supermultiplet in a bifundamental representation of $S U^{5}(3)$. Specifically, for an entry at the $i^{\text {th }}$ column and $j^{\text {th }}$ row we have a bifundamental representation of $S U_{i}(3) \times S U_{j}(3)$. We can arbitrarily assign the fundamental representation to the rows and the antifundamental representation to the columns. If $i=j$ the bifundamental is all in $S U_{i}(3)$ and hence is a singlet plus adjoint of $S U_{i}(3)$. Hence the complete set of chiral supermultiplets repre- 
sented by Table 1 is:

$$
\begin{aligned}
& {[(3, \overline{3}, 1,1,1)+(1,3, \overline{3}, 1,1)+(1,1,3, \overline{3}, 1)} \\
& +(1,1,1,3, \overline{3})+(\overline{3}, 1,1,1,3)] \\
& +2[(3,1, \overline{3}, 1,1)+(1,3,1, \overline{3}, 1)+(1,1,3,1, \overline{3}) \\
& +(\overline{3}, 1,1,3,1)+(1, \overline{3}, 1,1,3)] \\
& +[(1+8,1,1,1,1)+(1,1+8,1,1,1) \\
& +(1,1,1+8,1,1)+(1,1,1,1+8,1)+(1,1,1,1,1+8)]
\end{aligned}
$$

\begin{tabular}{|c||c|c|c|c|c|}
\hline$M_{122}^{5}$ & 1 & $\alpha$ & $\alpha^{2}$ & $\alpha^{3}$ & $\alpha^{4}$ \\
\hline \hline 1 & $\times$ & $\times$ & $\times \times$ & & \\
\hline$\alpha$ & & $\times$ & $\times$ & $\times \times$ & \\
\hline$\alpha^{2}$ & & & $\times$ & $\times$ & $\times \times$ \\
\hline$\alpha^{3}$ & $\times \times$ & & & $\times$ & $\times$ \\
\hline$\alpha^{4}$ & $\times$ & $\times \times$ & & & $\times$ \\
\hline
\end{tabular}

Table 1: Matter content for the model $M_{122}^{5}$. The $\times \times$ entry at the $\left(1, \alpha^{2}\right)$ position means the model contains $2(3,1, \overline{3}, 1,1)$ of $S U^{5}(3)$, etc. The diagonal entries are $(8+1,1,1,1,1)$, etc.

A vacuum expectation value $(V E V)$ for $(3, \overline{3}, 1,1,1)$ breaks the symmetry to $S U_{D}(3) \otimes S U_{3}(3) \otimes S U_{4}(3) \otimes S U_{5}(3)$ and a further $V E V$ for $(1,3, \overline{3}, 1)$ breaks the symmetry to $S U_{D}(3) \otimes$ $S U_{D^{\prime}}(3) \otimes S U_{5}(3)$. Identifiny $S U_{C}(3)$ with $S U_{D}(3)$, embedding $S U_{L}(2)$ in $S U_{D^{\prime}}(3)$ and $U_{Y}(1)$ partially in $S U_{5}(3)$ and partially in $S U_{D^{\prime}}(3)$ gives an initial value of $\sin ^{2} \theta_{W}=\frac{2}{7}=.286$, and implies a unification scale around $2 \times 10^{7} \mathrm{GeV}$.

The remaining chiral multiplets are

$$
3[(3, \overline{3}, 1)+(1,3, \overline{3})+(\overline{3}, 1,3)]
$$

We have sufficient octets to continue the symmetry breaking all the way to $S U(3) \otimes S U(2) \otimes U(1)$, and so arrive at the MSSM with three families (plus additional vector-like matter that is heavy and therefore not in the low energy spectrum). 
Before analyzing more models in detail, it is useful to tabulate the possible model for each value of $n$. To this end, note we always have a proper embedding (i.e., $\left.\mathbf{6}=\mathbf{6}^{*}\right)$ for $\mathbf{4}=\left(\mathbf{1}, \alpha^{i}, \alpha^{j}, \alpha^{k}\right)$ when $i+j+k=n$. To show this we use the fact that the conjugate model has $i \rightarrow i^{\prime}=n-i, j \rightarrow j^{\prime}=n-j$ and $k \rightarrow k^{\prime}=n-k$. Summing we find $i^{\prime}+j^{\prime}+k^{\prime}=3 n-(i+j+k)=2 n$. From $\mathbf{6}=(\mathbf{4} \otimes \mathbf{4})_{\text {antisym }}$ we find $\mathbf{6}=\left(\alpha^{i}, \alpha^{j}, \alpha^{k}, \alpha^{j+k}, \alpha^{i+k}, \alpha^{i+j}\right)$, but $i+j=n-k=k^{\prime}$. Likewise $i+k=j^{\prime}$ and $j+k=i^{\prime}$ so $\mathbf{6}=\left(\alpha^{i}, \alpha^{j}, \alpha^{k}, \alpha^{i^{\prime}}, \alpha^{j^{\prime}}, \alpha^{k^{\prime}}\right)$ and this is $\mathbf{6}^{*}$ up to an automorphism which is sufficient to provide vectorlike matter in this sector in the non-SUSY models and here provide a proper embedding. Models with $i+j+k=n$ (we will call these partition models) are always chiral, with total chirality $\chi=3 N^{2} n$ except in the case where $n$ is even and one of $i, j$, or $k$ is $n / 2$ where $\chi=2 N^{2} n$. (No more than one of $i, j$, and $k$ can be $n / 2$ since they add to $n$ and are all positive.) This immediately gives us a lower bound on the number of chiral models at fixed $n$. It is the the number of partitions of $n$ into three non-negative integers. There is another class of models with $i^{\prime}=k$ and $j^{\prime}=j^{2}$, and total chirality $\chi=N^{2} n$; for example a $Z_{9}$ orbifold with $\mathbf{4}=\left(\mathbf{1}, \alpha^{3}, \alpha^{3}, \alpha^{6}\right)$. And there are a few other sporadically occurring cases like $M_{124}^{6}$, which typically have reduced total chirality, $\chi<3 N^{2} n$.

We now tabulate all the $Z_{n}$ orbifold models up to $n=12$ along with the total chirality of each model, (see Table 2). 


\begin{tabular}{|c||c|c|c|}
\hline$n$ & $\mathbf{4}$ & $\chi / N^{2}$ & comment \\
\hline \hline 3 & $(\mathbf{1}, \alpha, \alpha, \alpha)$ & 9 & $i+j+k=3 ;$ one model $(i=j=k=1)$ \\
\hline 3 & $\left(\mathbf{1}, \alpha, \alpha, \alpha^{2}\right)^{*}$ & 3 & \\
\hline 4 & $\left(\mathbf{1}, \alpha, \alpha, \alpha^{2}\right)$ & 8 & $i+j+k=4 ;$ one model \\
\hline 5 & $\left(\mathbf{1}, \alpha^{i}, \alpha^{j}, \alpha^{k}\right)$ & 15 & $i+j+k=5 ; 2$ models \\
\hline 6 & $\left(\mathbf{1}, \alpha^{i}, \alpha^{j}, \alpha^{k}\right)$ & 12 & $i+j+k=6 ; 3$ models \\
\hline 6 & $\left(\mathbf{1}, \alpha, \alpha^{2}, \alpha^{4}\right)^{*}$ & 6 & \\
\hline 6 & $\left(\mathbf{1}, \alpha^{2}, \alpha^{2}, \alpha^{4}\right)^{*}$ & 6 & \\
\hline 7 & $\left(\mathbf{1}, \alpha^{i}, \alpha^{j}, \alpha^{k}\right)$ & 21 & $i+j+k=7 ; 4$ models \\
\hline 8 & $\left(\mathbf{1}, \alpha^{i}, \alpha^{j}, \alpha^{k}\right)$ & $\leq 24$ & $i+j+k=8 ; 5$ models \\
\hline 9 & $\left(\mathbf{1}, \alpha^{i}, \alpha^{j}, \alpha^{k}\right)$ & 27 & $i+j+k=9 ; 7$ models \\
\hline 9 & $\left(\mathbf{1}, \alpha, \alpha^{4}, \alpha^{7}\right)^{*}$ & 27 & \\
\hline 9 & $\left(\mathbf{1}, \alpha^{3}, \alpha^{3}, \alpha^{6}\right)^{*}$ & 9 & \\
\hline 10 & $\left(\mathbf{1}, \alpha^{i}, \alpha^{j}, \alpha^{k}\right)$ & 30 & $i+j+k=10 ; 8$ models \\
\hline 11 & $\left(\mathbf{1}, \alpha^{i}, \alpha^{j}, \alpha^{k}\right)$ & 33 & $i+j+k=11 ; 10$ models \\
\hline 12 & $\left(\mathbf{1}, \alpha^{i}, \alpha^{j}, \alpha^{k}\right)$ & $\leq 36$ & $i+j+k=12 ; 12$ models \\
\hline 12 & $\left(\mathbf{1}, \alpha^{2}, \alpha^{4}, \alpha^{8}\right)^{*}$ & 12 & \\
\hline 12 & $\left(\mathbf{1}, \alpha^{4}, \alpha^{4}, \alpha^{8}\right)^{*}$ & 12 & \\
\hline
\end{tabular}

Table 2. All chiral $Z_{n}$ orbifold models with $n \leq 12$. Three of the $n=8$ models have $\chi / N^{2}=24$; the other two have $\chi / N^{2}=$ 16. Of the 12 models with $i+j+k=12$, three have models $\chi / N^{2}=24$ and the other nine have $\chi / N^{2}=36$. Of the 60 models 53 are partition models, while the remaining 7 models that do not satisfy $i+j+k=n$, are marked with an asterisk $\left(^{*}\right)$.

We have analyzed all the models for $Z_{6}$ orbifolds, and find only one of phenomenological interest. It is $M_{123}^{6}$, where the matter multiplets are shown in Table 3.

\begin{tabular}{|c||c|c|c|c|c|c|}
\hline$M_{123}^{6}$ & 1 & $\alpha$ & $\alpha^{2}$ & $\alpha^{3}$ & $\alpha^{4}$ & $\alpha^{5}$ \\
\hline \hline 1 & $\times$ & $\times$ & $\times$ & $\times$ & & \\
\hline$\alpha$ & & $\times$ & $\times$ & $\times$ & $\times$ & \\
\hline$\alpha^{2}$ & & & $\times$ & $\times$ & $\times$ & $\times$ \\
\hline$\alpha^{3}$ & $\times$ & & & $\times$ & $\times$ & $\times$ \\
\hline$\alpha^{4}$ & $\times$ & $\times$ & & & $\times$ & $\times$ \\
\hline$\alpha^{5}$ & $\times$ & $\times$ & $\times$ & & & $\times$ \\
\hline
\end{tabular}


Table 3: Chiral supermultiplets for the model $M_{123}^{6}$.

$V E V \mathrm{~s}$ for $(3, \overline{3}, 1,1,1,1),(1,1,3, \overline{3}, 1,1)$ and $(1,1,1,1,3, \overline{3})$ break the symmetry to $S U_{12}(3) \otimes S U_{34}(3) \otimes S U_{56}(3)$ where $S U_{12}(3)$ is the diagonal subgroup of $S U_{1}(3) \otimes S U_{2}(3)$, etc., and the remaining chirality resides in $3[(3, \overline{3}, 1)+(1,3, \overline{3})+(\overline{3}, 1,3)]$. Again, we have octets of all six initial $S U(3)$ s, so we can break to a three-family $M S S M$, but with $\sin ^{2} \theta_{W}=\frac{3}{8}$. There is no other pattern of $S S B$ that gives three families.

For $Z_{7}$, we again find only one model that can break to a three-family $M S S M$. It is $M_{133}^{7}$, with matter shown in Table 4.

\begin{tabular}{|c||c|c|c|c|c|c|c|}
\hline$M_{133}^{7}$ & 1 & $\alpha$ & $\alpha^{2}$ & $\alpha^{3}$ & $\alpha^{4}$ & $\alpha^{5}$ & $\alpha^{6}$ \\
\hline \hline 1 & $\times$ & $\times$ & & $\times \times$ & & & \\
\hline$\alpha$ & & $\times$ & $\times$ & & $\times \times$ & & \\
\hline$\alpha^{2}$ & & & $\times$ & $\times$ & & $\times \times$ & \\
\hline$\alpha^{3}$ & & & & $\times$ & $\times$ & & $\times \times$ \\
\hline$\alpha^{4}$ & $\times \times$ & & & & $\times$ & $\times$ & \\
\hline$\alpha^{5}$ & & $\times \times$ & & & & $\times$ & $\times$ \\
\hline$\alpha^{6}$ & $\times$ & & $\times \times$ & & & & $\times$ \\
\hline
\end{tabular}

Table 4: Chiral supermultiplets for the model $M_{133}^{7}$.

First $V E V \mathrm{~s}$ for $(3, \overline{3}, 1,1,1,1,1)$ and $(1,1,3, \overline{3}, 1,1,1)$ breaks the symmetry to $S U_{12}(3) \otimes S U_{34}(3) \otimes S U_{5}(3) \otimes S U_{6}(3) \otimes S U_{7}(3)$. Then a $V E V$ for $(1,1,1,3, \overline{3})$ breaks this to $S U_{12}(3) \otimes S U_{34}(3) \otimes$ $S U_{5}(3) \otimes S U_{67}(3)$, and leaves the following multiplets chiral

$$
\begin{aligned}
& (3, \overline{3}, 1,1)+(1,3, \overline{3}, 1)+(1,1,3, \overline{3})+(\overline{3}, 1,1,3) \\
& +2[(3, \overline{3}
\end{aligned}
$$

Finally, a $V E V$ for $(1,3, \overline{3}, 1)$ yields the $M S S M$ with three chiral families. Identifying $S U_{C}(3)$ with $S U_{12}(3)$ and embedding $S U_{L}(2)$ in $S U_{67}(3)$ and $U_{Y}(1)$ in $S U_{345}(3)$ gives $\sin ^{2} \theta_{W}=\frac{7}{22}=$ .318 and implies a unification scale around $10^{10} \mathrm{GeV}$. 
The $n>7$ models can be analyzed in a similar manner. The total number of models grows with $n$. There are also potentially interesting examples for $N>4$. Although we have not made a systematic study of the models with $n \geq 8$, we close with a rather compact example of a three-family $M S S M$ at $n=9$. The model is $M_{123}^{6}$ with matter given in Table 5 .

\begin{tabular}{|c||c|c|c|c|c|c|c|c|c|}
\hline$M_{333}^{9}$ & 1 & $\alpha$ & $\alpha^{2}$ & $\alpha^{3}$ & $\alpha^{4}$ & $\alpha^{5}$ & $\alpha^{6}$ & $\alpha^{7}$ & $\alpha^{8}$ \\
\hline \hline 1 & $\times$ & & & $\times \times \times$ & & & & & \\
\hline$\alpha$ & & $\times$ & & & $\times \times \times$ & & & & \\
\hline$\alpha^{2}$ & & & $\times$ & & & $\times \times \times$ & & & \\
\hline$\alpha^{3}$ & & & & $\times$ & & & $\times \times \times$ & & \\
\hline$\alpha^{4}$ & & & & & $\times$ & & & $\times \times \times$ & \\
\hline$\alpha^{5}$ & & & & & & $\times$ & & & $\times \times \times$ \\
\hline$\alpha^{6}$ & $\times \times \times$ & & & & & & $\times$ & & \\
\hline$\alpha^{7}$ & & $\times \times \times$ & & & & & & $\times$ & \\
\hline$\alpha^{8}$ & & & $\times \times \times$ & & & & & & $\times$ \\
\hline
\end{tabular}

Table 5: Chiral supermultiplets for the model $M_{333}^{9}$.

$V E V$ s for the octets of $S U_{k}(3)$, where $k=2,3,5,6,8$, and 9 breaks the symmetry to $S U_{1}(3) \otimes S U_{4}(3) \otimes S U_{7}(3)$. (Each chiral supermultiplet of representation $R$ contains one chiral fermion multiplet in representation $R$, and two scalar (we need not distinguish scalars from pseudoscalars here) multiplets in representation $R$. Therefore, there are two scalar octets for each $S U_{k}(3)$. When one octet of $S U_{k}(3)$ is given a $V E V$, gauge freedom can be used to diagonalize that $V E V$. However, there is not enough gauge freedom left to diagonalize the $V E V$ of the second octet of the same $S U_{k}(3)$. Therefore $S U_{k}(3)$ can be broken completely by $S U_{k}(3)$ s for the two octets). The chirality remaining after this octet breaking is $3[(3, \overline{3}, 1)+(1,3, \overline{3})+(\overline{3}, 1,3)]$. Further symmetry breaking via single octets of $S U_{3}(3)$ and $S U_{7}(3)$ leads us to the three-family $M S S M$, but with $\sin ^{2} \theta_{W}=\frac{3}{8}$. Note that any model of the type $M_{\frac{n}{3} \frac{n}{3} \frac{n}{3}}^{n}$ can be handled this way, and can lead to a three-family $M S^{3} S^{3} M$. Hence this provides an infinite class of three-family models. 
We have found 60 chiral $Z_{n}$ orbifolds for $n \leq 12$. A systematic search up through $n=7$ yields four models that can result in three-family minimal supersymmetric standard models. They are $M_{111}^{3}, M_{122}^{5}, M_{123}^{6}$, and $M_{133}^{7}$. We suspect there are many more models with sensible phenomenology at larger $n$, and we have pointed out one example $M_{333}^{9}$, which is particularly simple in its spontaneous symmetry breaking, and is also a member of an infinite series of models $M_{\frac{n}{3} \frac{n}{3} \frac{n}{3}}^{n}$, which all can lead to three-family $M S S M$ s. Orbifolded $A d S / C F T$ models hold great promise for testing string theory not far above the the $T e V$ scale, and they have inspired models [161] with phenomenology ranging from light magnetic monopoles [34] to an anomalous muon magnetic moment [193]. They have also provided a check on higher loop $\beta$ functions [191], and raised interesting cosmological questions [140]. 


\subsection{Non abelian model building}

We shall present what we believe is the minimal three-family $A d S / C F T$ model compactified on a nonabelian orbifold $S^{5} /(Q \times$ $\left.Z_{3}\right)$. Nontrivial irreps of the discrete nonabelian group $Q \times Z_{3}$ are identified with the 4 of $S U(4) R$ symmetry to break all supersymmetries, and the scalar content of the model is sufficient to break the gauge symmetry to the standard model. According to the conformality hypothesis the progenitor $S U(4)^{3} \times S U(2)^{12}$ theory becomes conformally invariant at an infra-red fixed point of the renormalization group.

\subsubsection{Non-Abelian non-SUSY models}

For construction of quiver gauge theories from orbifolds, please refer back again to Section 2 .

It was conjectured in [14] that at least a subset of the resultant nonsupersymmetric $\mathcal{N}=0$ theories are conformal even for finite $N$. Some first steps to check this idea were made in [174]. Model-building based on abelian $\Gamma$ was studied further in [175-177], arriving in [177] at an $S U(3)^{7}$ model based on $\Gamma=Z_{7}$ which has three families of chiral fermions, a correct value for $\sin ^{2} \theta$ and a conformal scale $\sim 10 \mathrm{TeV}$.

The case of non-abelian orbifolds bases on non-abelian $\Gamma$ is somewhat more mathematically sophisticated. However, we shall show here that it can be handled equally as systematically as the abelian case and leads to richer structures and interesting results.

We consider all non-abelian discrete groups of order $g<32$. These are described in detail in [180,194-198]. There are exactly 45 such non-abelian groups. Because the gauge group arrived at by this construction [175] is $\otimes_{i} U\left(N d_{i}\right)$ where $d_{i}$ are the dimensions of the irreducible representations of $\Gamma$, one can expect to arrive at models such as the Pati-Salam $S U(4) \times S U(2) \times S U(2)$ 
type [164] by choosing $N=2$ and combining two singlets and a doublet in the 4 of $S U(4)$. Indeed we shall show that such an accommodation of the standard model is possible by using a non-abelian $\Gamma$.

The procedures for building a model within such a conformality approach are: (1) Choose $\Gamma$; (2) Choose a proper embedding $\Gamma \subset S U(4)$ by assigning the components of the 4 of $S U(4)$ to irreps of $\Gamma$, while at the same time ensuring that the $\mathbf{6}$ of $S U(4)$ is real; (3) Choose $N$, in the gauge group $\otimes_{i} S U\left(N d_{i}\right)$. (4) Analyse the patterns of spontaneous symmetry breaking.

In the present study we shall choose $N=2$ and aim at the gauge group $S U(4) \times S U(2) \times S U(2)$. To obtain chiral fermions, it is necessary [173] that the $\mathbf{4}$ of $S U(4)$ be complex $\boldsymbol{4} \neq \mathbf{4}^{*}$. Actually this condition is not quite sufficient to ensure chirality in the present case because of the pseudoreality of $S U(2)$. We must ensure that the $\mathbf{4}$ is not just pseudoreal.

This last condition means that many of our 45 candidates for $\Gamma$ do not lead to chiral fermions. For example, $\Gamma=Q_{2 n} \subset S U(2)$ has irreps of appropriate dimensionalities for our purpose but it will not sustain chiral fermions under $S U(4) \times S U(2) \times S U(2)$ because these irreps are all, like $S U(2)$, pseudoreal.\#3 Applying the rule that 4 must be neither real nor pseudoreal leaves a total of only 19 possible non-abelian discrete groups of order $g \leq 31$. The smallest group which avoids pseudoreality has order $g=16$ but gives only two families. The technical details of our systematic search will be postponed to a future publication. Here we shall present only the simplest interesting non-abelian case which has $g=24$ and gives three chiral families in a PatiSalam-type model [164].

Before proceeding to the details of the specific $g=24$ case, it is worth reminding the reader that the Conformal Field Theory (CFT) that it exemplifies should be free of all divergences, even logarithmic ones, if the conformality conjecture is correct,

\footnotetext{
${ }^{\# 3}$ Note that were we using $N \geq 3$ then a pseudoreal 4 would give chiral fermions.
} 
and be completely finite. Further the theory is originating from a superstring theory in a higher-dimension (ten) and contains gravity [199-214] by compactification of the higher-dimensional graviton already contained in that superstring theory. In the CFT as we derive it, gravity is absent because we have not kept these graviton modes - of course, their influence on high-energy physics experiments is generally completely negligible unless the compactification scale is "large" [199,214]; here we shall neglect the effects of gravity.

To motivate our model it is instructive to comment on the choice of $\Gamma$ and on the choice of embedding.

If we embed only four singlets of $\Gamma$ in the 4 of $S U(4)$ then this has the effect of abelianizing $\Gamma$ and the gauge group obtained in the chiral sector of the theory is $S U(N)^{q}$. These cases can be interesting but have already been studied $[175,176]$. Thus, we require at least one irrep of $\Gamma$ to have $d_{i} \geq 2$ in the embedding.

The only $\Gamma$ of order $g \leq 31$ with a 4 is $Z_{5} \tilde{\times} Z_{4}$ and this embedding leads to a non-chiral theory. This leaves only embeddings with two singlets and a doublet, a triplet and a singlet or two doublets.

The third of these choices leads to richer structures for low order $\Gamma$. Concentrating on them shows that of the chiral models possible, those from groups of low order result in an insufficient number (below three) of chiral families.

The first group that can lead to exactly three families occurs at order $g=24$ and is $\Gamma=Z_{3} \times Q$ where $Q\left(\equiv Q_{4}\right)$ is the group of unit quarternions which is the smallest dicyclic group $Q_{2 n}$.

There are several potential models due to the different choices for the $\mathbf{4}$ of $S U(4)$ but only the case $\mathbf{4}=\left(1 \alpha, 1^{\prime}, 2 \alpha\right)$ leads to three families so let us describe this in some detail:

Since $Q \times Z_{3}$ is a direct product group, we can write the irreps as $R_{i} \otimes \alpha^{a}$ where $R_{i}$ is a $Q$ irrep and $\alpha^{a}$ is a $Z_{3}$ irrep. We write $Q$ irreps as $1,1^{\prime}, 1^{\prime \prime}, 1^{\prime \prime \prime}, 2$ while the irreps of $Z_{3}$ are all singlets which we call $\alpha, \alpha^{2}, \alpha^{3}=1$. Thus $Q \times Z_{3}$ has fiveteen irreps in 
all and the gauge group will be of Pati-Salam type for $N=2$.

If we wish to break all supersymmetry, the $\mathbf{4}$ may not contain the trivial singlet of $\Gamma$. Due to permutational symmetry among the singlets it is sufficiently general to choose $\mathbf{4}=$ $\left(1 \alpha^{a_{1}}, 1^{\prime} \alpha^{a_{2}}, 2 \alpha^{a_{3}}\right)$ with $a_{1} \neq 0$.

To fix the $a_{i}$ we note that the scalar sector of the theory which is generated by the $\mathbf{6}$ of $S U(4)$ can be used as a constraint since the 6 is required to be real. This leads to $a_{1}+a_{2}=$ $-2 a_{3}(\bmod 3)$. Up to permutations in the chiral fermion sector the most general choice is $a_{1}=a_{3}=+1$ and $a_{2}=0$. Hence our choice of embedding is

$$
\mathbf{4}=\left(1 \alpha, 1^{\prime}, 2 \alpha\right)
$$

with

$$
\mathbf{6}=\left(1^{\prime} \alpha, 2 \alpha, 2 \alpha^{2}, 1^{\prime} \alpha^{2}\right)
$$

which is real as required.

We are now in a position to summarize the particle content of the theory. The fermions are given by

$$
\sum_{I} 4 \times R_{I}
$$

where the $R_{I}$ are all the irreps of $\Gamma=Q \times Z_{3}$. This is:

$$
\begin{aligned}
& \sum_{i=1}^{3}\left[\left(2_{1} \alpha^{i}, 2_{2} \alpha^{i}\right)+\left(2{ }_{3} \alpha^{i}, 2_{4} \alpha^{i}\right)+\left(2{ }_{2} \alpha^{i}, 2_{1} \alpha^{i}\right)+\left(2{ }_{4} \alpha^{i}, 2_{3} \alpha^{i}\right)+\left(4 \alpha^{i}, \overline{4} \alpha^{i}\right)\right] \\
& \quad+\sum_{i=1}^{3} \sum_{a=1}^{4}\left[\left(2_{a} \alpha^{i}, 2_{a} \alpha^{i+1}\right)+\left(2{ }_{a} \alpha^{i}, 4 \alpha^{i+1}\right)+\left(\overline{4} \alpha^{i}, 2_{a} \alpha^{i+1}\right)\right]
\end{aligned}
$$

It is convenient to represent the chiral portions of these in a given diagram (see Figure 1).

The scalars are given by

$$
\sum_{I} 6 \times R_{I}
$$


and are:

$$
\begin{gathered}
\sum_{i=1}^{3} \sum_{j=1(j \neq i)}^{3}\left[\left(2 \alpha_{1} \alpha^{i}, 2_{2} \alpha^{j}\right)+\left(2{ }_{2} \alpha^{i}, 2{ }_{1} \alpha^{j}\right)+\left(2{ }_{3} \alpha^{i}, 2{ }_{4} \alpha^{j}\right)+\left(2{ }_{4} \alpha^{i}, 2{ }_{3} \alpha^{j}\right)\right. \\
\left.+\left(2{ }_{2} \alpha^{i}, 2_{1} \alpha^{i}\right)+\left(2{ }_{4} \alpha^{i}, 2{ }_{3} \alpha^{i}\right)\right] \\
+\sum_{i=1}^{3} \sum_{j=1(j \neq i)}^{3}\left\{\sum_{a=1}^{4}\left[\left(22_{a} \alpha^{i}, 4 \alpha^{j}\right)+\left(\overline{4} \alpha^{i}, 2{ }_{a} \alpha^{j}\right)\right]+\left(4 \alpha^{i}, \overline{4} \alpha^{i}\right)\right\}
\end{gathered}
$$

which is easily checked to be real.

The gauge group $S U(4)^{3} \times S U(2)^{12}$ with chiral fermions of Eq.(22) and scalars of Eq.(26) is expected to acquire confromal invariance at an infra-red fixed point of the renormalization group, as discussed in [14].

To begin our examination of the symmetry breaking we first observe that if we break the three $S U(4)$ s to the totally diagonal $S U(4)$, then chirality in the fermionic sector is lost. To avoid this we break $S U_{1}(4)$ completely and then break $S U_{\alpha}(4) \times S U_{\alpha^{2}}(4)$ to its diagonal subgroup $S U_{D}(4)$. The first of these steps can be achieved with VEVs of the form $\left[\left(4_{1}, 2_{b} \alpha^{k}\right)+h . c.\right]$ where we are free to choose $b$, but $k$ must be 1 or 2 since there are no $\left(4_{1}, 2_{b} \alpha^{k=0}\right)$ scalars. The second step requires an $S U_{D}(4)$ singlet VEV from $\left(\overline{4}_{\alpha}, 4_{\alpha^{2}}\right)$ and/or $\left(4_{\alpha}, \overline{4}_{\alpha^{2}}\right)$. Once we make a choice for $b$ (we take $b=4$ ), the remaining chiral fermions are, in an intuitive notation:

$$
\sum_{a=1}^{3}\left[\left(2_{a} \alpha, 1,4_{D}\right)+\left(1,2_{a} \alpha^{-1}, \overline{4_{D}}\right)\right]
$$

which has the same content as as a three family Pati-Salam model, though with a separate $S U_{L}(2) \times S U_{R}(2)$ per family.

To further reduce the symmetry we must arrange to break to a single $S U_{L}(2)$ and a single $S U_{R}(2)$. This is achieved by modifying step one where $S U_{1}(4)$ was broken. Consider the block diagonal decomposition of $S U_{1}(4)$ into $S U_{1 L}(2) \times S U_{1 R}(2)$. 
The representations $\left(2{ }_{a} \alpha, 4_{1}\right)$ and $\left(2 \alpha_{a} \alpha^{-1}, 4_{1}\right)$ then decompose as

$$
\left(2_{a} \alpha, 4_{1}\right) \rightarrow\left(2_{a} \alpha, 2,1\right)+\left(2_{a} \alpha, 1,2\right)
$$

and

$$
\left(2_{a} \alpha^{-1}, 4_{1}\right) \rightarrow\left(2{ }_{a} \alpha^{-1},, 2,1\right)+\left(2_{a} \alpha^{-1}, 1,2\right) .
$$

Now if we give $V E V s$ of equal magnitude to the $\left(2_{a} \alpha, 2,1\right)$, $a=1,2,3$, and equal magnitude $V E V s$ to the $\left(2_{a} \alpha^{-1}, 1,2\right)$ $a=1,2,3$, we break $S U_{1 L}(2) \times \prod_{a=1}^{3} S U\left(2_{a} \alpha\right)$ to a single $S U_{L}(2)$ and we break $S U_{1 R}(2) \times \prod_{a=1}^{3} S U\left(2_{a} \alpha\right)$ to a single $S U_{R}(2)$. Finally, $V E V s$ for $\left(2{ }_{4} \alpha, 2,1\right)$ and $\left(2{ }_{4} \alpha, 1,2\right)$ as well as $\left(2 \alpha_{4} \alpha^{-1}, 2,1\right)$ and $\left(2{ }_{4} \alpha^{-1}, 1,2\right)$ insures that both $S U\left(2{ }_{4} \alpha\right)$ and $S U\left(2_{4} \alpha^{-1}\right)$ are broken and that only three families remain chiral. The final set of chiral fermions is then $3[(2,1,4)+(1,2, \overline{4})]$ with gauge symmetry $S U_{L}(2) \times S U_{R}(2) \times S U_{D}(4)$.

To achieve the final reduction to the standard model, an adjoint VEV from $\left(\overline{4}_{\alpha}, 4_{\alpha^{2}}\right)$ and/or $\left(4_{\alpha}, \overline{4}_{\alpha^{2}}\right)$ is used to break $S U_{D}(4)$ to the $S U(3) \times U(1)$, and a right handed doublet is used to break $S U_{R}(2)$.

While this completes our analysis of symmetry breaking, it is worthwhile noting the degree of constraint imposed on the symmetry and particle content of a model as the number of irreps $N_{R}$ of the discrete group $\Gamma$ associated with the choice of orbifold changes. The number of guage groups grows linearly in $N_{R}$, the number of scalar irreps grows roughly quadratically with $N_{R}$, and the chiral fermion content is highly $\Gamma$ dependent. If we require the minimal $\Gamma$ that is large enough for the model generated to contain the fermions of the standard model and have sufficient scalars to break the symmetry to that of the standard model, then $\Gamma=Q \times Z_{3}$ appears to be that minimal choice $[178,180]$.

Although a decade ago the chances of testing string theory seemed at best remote, recent progress has given us hope that 
such tests may indeed be possible in AdS/CFTs. The model provided here demonstrates the standard model can be accomodated in these theories and suggests the possibility of a rich spectrum of new physics just around the TeV corner. 


\subsection{Non-Abelian Groups with order $g \leq 31$}

From any good textbook on finite groups [195-198] we may find a tabulation of the number of finite groups as a function of the order $\mathrm{g}$, the number of elements in the group. Up to order 31 there is a total of 93 different finite groups of which slightly over one half (48) are abelian.

Amongst finite groups, the non-abelian examples have the advantage of non-singlet irreducible representations which can be used to inter-relate families. Which such group to select is based on simplicity: the minimum order and most economical use of representations.

Let us first dispense with the abelian groups. These are all made up from the basic unit $Z_{p}$, the order $\mathrm{p}$ group formed from the $p^{\text {th }}$ roots of unity. It is important to note that the product $Z_{p} \times Z_{q}$ is identical to $Z_{p q}$ if and only if $\mathrm{p}$ and $\mathrm{q}$ have no common prime factor.

If we write the prime factorization of $g$ as:

$$
g=\prod_{i} p_{i}^{k_{i}}
$$

where the product is over primes, it follows that the number $N_{a}(g)$ of inequivalent abelian groups of order $\mathrm{g}$ is given by:

$$
N_{a}(g)=\prod_{k_{i}} P\left(k_{i}\right)
$$

where $P(x)$ is the number of unordered partitions of $x$. For example, for order $g=144=2^{4} 3^{2}$ the value would be $N_{a}(144)=$ $P(4) P(2)=5 \times 2=10$. For $g \leq 31$ it is simple to evaluate $N_{a}(g)$ by inspection. $N_{a}(g)=1$ unless g contains a nontrivial power $\left(k_{i} \geq 2\right)$ of a prime. These exceptions are: $N_{a}(g=$ $4,9,12,18,20,25,28)=2 ; N_{a}(8,24,27)=3 ;$ and $N_{a}(16)=5$. This confirms that:

$$
\sum_{g=1}^{31} N_{a}(g)=48
$$


We do not consider the abelian cases further in this section.

Of the nonabelian finite groups, the best known are perhaps the permutation groups $S_{N}$ (with $N \geq 3$ ) of order $N$ ! The smallest non-abelian finite group is $S_{3}\left(\equiv D_{3}\right)$, the symmetry of an equilateral triangle with respect to all rotations in a three dimensional sense. This group initiates two infinite series, the $S_{N}$ and the $D_{N}$. Both have elementary geometrical significance since the symmetric permutation group $S_{N}$ is the symmetry of the N-plex in $\mathrm{N}$ dimensions while the dihedral group $D_{N}$ is the symmetry of the planar $\mathrm{N}$-agon in 3 dimensions. As a family symmetry, the $S_{N}$ series becomes uninteresting rapidly as the order and the dimensions of the representions increase. Only $S_{3}$ and $S_{4}$ are of any interest as symmetries associated with the particle spectrum [215], also the order (number of elements) of the $S_{N}$ groups grow factorially with $\mathrm{N}$. The order of the dihedral groups increase only linearly with $\mathrm{N}$ and their irreducible representations are all one- and two- dimensional. This is reminiscent of the representations of the electroweak $S U(2)_{L}$ used in Nature.

Each $D_{N}$ is a subgroup of $O(3)$ and has a counterpart double dihedral (also known as dicyclic) group $Q_{2 N}$, of order $4 N$, which is a subgroup of the double covering $S U(2)$ of $O(3)$.

With only the use of $D_{N}, Q_{2 N}, S_{N}$ and the tetrahedral group $\mathrm{T}$ ( of order 12, the even permutations subgroup of $S_{4}$ ) we find 32 of the 45 nonabelian groups up to order 31, either as simple groups or as products of simple nonabelian groups with abelian groups: (Note that $D_{6} \simeq Z_{2} \times D_{3}, D_{10} \simeq Z_{2} \times D_{5}$ and $D_{14} \simeq$ $Z_{2} \times D_{7}$ ) Some of these groups are firmiliar from crystalography and chemistry, but the nonabelian groups that do not embed in in $S U(2)$ are less likely to have seen wide usage. 


\begin{tabular}{||c||c||}
\hline $\mathrm{g}$ & \\
\hline 6 & $D_{3} \equiv S_{3}$ \\
\hline 8 & $D_{4}, Q=Q_{4}$ \\
\hline 10 & $D_{5}$ \\
\hline 12 & $D_{6}, Q_{6}, T$ \\
\hline 14 & $D_{7}$ \\
\hline 16 & $D_{8}, Q_{8}, Z_{2} \times D_{4}, Z_{2} \times Q$ \\
\hline 18 & $D_{9}, Z_{3} \times D_{3}$ \\
\hline 20 & $D_{10}, Q_{10}$ \\
\hline 22 & $D_{11}$ \\
\hline 24 & $D_{12}, Q_{12}, Z_{2} \times D_{6}, Z_{2} \times Q_{6}, Z_{2} \times T$, \\
\hline & $Z_{3} \times D_{4}, Z_{3} \times Q, Z_{4} \times D_{3}, S_{4}$ \\
\hline 26 & $D_{13}$ \\
\hline 28 & $D_{14}, Q_{14}$ \\
\hline 30 & $D_{15}, D_{5} \times Z_{3}, D_{3} \times Z_{5}$ \\
\hline
\end{tabular}

There remain thirteen others formed by twisted products of abelian factors. Only certain such twistings are permissable, namely (completing all $g \leq 31$ )

\begin{tabular}{||c||c||}
\hline $\mathrm{g}$ & \\
\hline 16 & $Z_{2} \tilde{\times} Z_{8}$ (two, excluding $\left.D_{8}\right), Z_{4} \tilde{\times} Z_{4}, Z_{2} \tilde{\times}\left(Z_{2} \times Z_{4}\right)$ (two) \\
\hline 18 & $Z_{2} \tilde{\times}\left(Z_{3} \times Z_{3}\right)$ \\
\hline 20 & $Z_{4} \tilde{\times} Z_{5}$ \\
\hline 21 & $Z_{3} \tilde{\times} Z_{7}$ \\
\hline 24 & $Z_{3} \tilde{\times} Q, Z_{3} \tilde{\times} Z_{8}, Z_{3} \tilde{\times} D_{4}$ \\
\hline 27 & $Z_{9} \tilde{\times} Z_{3}, Z_{3} \tilde{\times}\left(Z_{3} \times Z_{3}\right)$ \\
\hline
\end{tabular}

It can be shown that these thirteen exhaust the classification of all inequivalent finite groups up to order thirty-one [198].

Of the 45 nonabelian groups, the dihedrals $\left(D_{N}\right)$ and double dihedrals $\left(Q_{2 N}\right)$, of order $2 \mathrm{~N}$ and $4 \mathrm{~N}$ respectively, form the simplest sequences. In particular, they fall into subgroups of $O(3)$ 
and $S U(2)$ respectively, the two simplest nonabelian continuous groups.

For the $D_{N}$ and $Q_{2 N}$, the multiplication tables, as derivable from the character tables, are simple to express in general. $D_{N}$, for odd $\mathrm{N}$, has two singlet representations $1,1^{\prime}$ and $m=(N-$ 1)/2 doublets $2_{(j)}(1 \leq j \leq m)$. The multiplication rules are:

$$
\begin{gathered}
1^{\prime} \times 1^{\prime}=1 ; \quad 1^{\prime} \times 2_{(j)}=2_{(j)} \\
2_{(i)} \times 2_{(j)}=\delta_{i j}\left(1+1^{\prime}\right)+2_{(\min [i+j, N-i-j])}+\left(1-\delta_{i j}\right) 2_{(|i-j|)}
\end{gathered}
$$

For even $\mathrm{N}, D_{N}$ has four singlets $1,1^{\prime}, 1^{\prime \prime}, 1^{\prime \prime \prime}$ and $(m-1)$ doublets $2_{(j)}(1 \leq j \leq m-1)$ where $m=N / 2$ with multiplication rules:

$$
\begin{gathered}
1^{\prime} \times 1^{\prime}=1^{\prime \prime} \times 1^{\prime \prime}=1^{\prime \prime \prime} \times 1^{\prime \prime \prime}=1 \\
1^{\prime} \times 1^{\prime \prime}=1^{\prime \prime \prime} ; 1^{\prime \prime} \times 1^{\prime \prime \prime}=1^{\prime} ; 1^{\prime \prime \prime} \times 1^{\prime}=1^{\prime \prime} \\
1^{\prime} \times 2_{(j)}=2_{(j)} \\
1^{\prime \prime} \times 2_{(j)}=1^{\prime \prime \prime} \times 2_{(j)}=2_{(m-j)} \\
2_{(j)} \times 2_{(k)}=2_{|j-k|}+2_{(\min [j+k, N-j-k])}
\end{gathered}
$$

(if $k \neq j,(m-j))$

$$
2_{(j)} \times 2_{(j)}=2_{(\min [2 j, N-2 j])}+1+1^{\prime}
$$

(if $j \neq m / 2$ )

$$
2_{(j)} \times 2_{(m-j)}=2_{|m-2 j|}+1^{\prime \prime}+1^{\prime \prime \prime}
$$

(if $j \neq m / 2$ )

$$
2_{m / 2} \times 2_{m / 2}=1+1^{\prime}+1^{\prime \prime}+1^{\prime \prime \prime}
$$

This last rule is possible only if $\mathrm{m}$ is even and hence if $\mathrm{N}$ is divisible by four. 
For $Q_{2 N}$, there are four singlets $1,1^{\prime}, 1^{\prime \prime}, 1^{\prime \prime \prime}$ and $(N-1)$ doublets $2_{(j)}(1 \leq j \leq(N-1))$. The singlets have the multiplication rules:

$$
\begin{gathered}
1 \times 1=1^{\prime} \times 1^{\prime}=1 \\
1^{\prime \prime} \times 1^{\prime \prime}=1^{\prime \prime \prime} \times 1^{\prime \prime \prime}=1^{\prime} \\
1^{\prime} \times 1^{\prime \prime}=1^{\prime \prime \prime} ; 1^{\prime \prime \prime} \times 1^{\prime}=1^{\prime \prime}
\end{gathered}
$$

for $N=(2 k+1)$ but are identical to those for $D_{N}$ when $\mathrm{N}=$ $2 \mathrm{k}$.

The products involving the $2_{(j)}$ are identical to those given for $D_{N}$ (N even) above.

This completes the multiplication rules for 19 of the 45 groups. As they are not available in the literature, and somewhat tedious to work out, we have provided the complete multiplication tables for all the nonabelian groups with order $g \leq 31$ in [180]. 
Mathematical Theorem:

A Pseudoreal 4 of $S U(4)$ Cannot Yield Chiral Fermions.

In [173] it was proved that if the embedding in $S U(4)$ is such that the 4 is real: $\mathbf{4}=\mathbf{4}^{*}$, then the resultant fermions are always non-chiral. It was implied there that the converse holds, that if 4 is complex, $4=\mathbf{4}^{*}$, then the resulting fermions are necessarily chiral. Actually for $\Gamma \subset S U(2)$ one encounters the intermediate possibility that the $\mathbf{4}$ is pseudoreal. In the present section we shall show that if $\mathbf{4}$ is pseudoreal then the resultant fermions are necessarily non-chiral. The converse now holds: if the $\mathbf{4}$ is neither real nor pseudoreal then the resutant fermions are chiral.

For $\Gamma \subset S U(2)$ it is important that the embedding be contained within the chain $\Gamma \subset S U(2) \subset S U(4)$ otherwise the embedding is not a consistent one. One way to see the inconsistency is to check the reality of the $\mathbf{6}=(\mathbf{4} \otimes \mathbf{4})_{\text {antisymmetric }}$. If $\mathbf{6} \neq \mathbf{6}^{*}$ then the embedding is clearly improper. To avoid this inconsistency it is sufficient to include in the $\mathbf{4}$ of $S U(4)$ only complete irreducible representations of $S U(2)$.

An explicit example will best illustrate this propriety constraint on embeddings. Let us consider $\Gamma=Q_{6}$, the dicyclic group of order $g=12$. This group has six inequivalent irreducible representations: $1,1^{\prime}, 1^{\prime \prime}, 1^{\prime \prime \prime}, 2_{1}, 2_{2}$. The $1,1^{\prime}, 2_{1}$ are real. The $1^{\prime \prime}$ and $1^{\prime \prime \prime}$ are a complex conjugate pair, The $2_{2}$ is pseudoreal. To embed $\Gamma=Q_{6} \subset S U(4)$ we must choose from the special combinations which are complete irreducible representations of $S U(2)$ namely $1,2=2_{2}, 3=1^{\prime}+2_{1}$ and $4=1^{\prime \prime}+1^{\prime \prime \prime}+2_{2}$. In this way the embedding either makes the 4 of $S U(4)$ real e.g. $4=1+1^{\prime}+2_{1}$ and the theorem of [173] applies, and nonchirality results, or the 4 is pseudoreal e.g. $4=2_{2}+2_{2}$. In this case one can check that the embedding is consistent because $(\mathbf{4} \otimes \mathbf{4})_{\text {antisymmetric }}$ is real. But it is equally easy to check that the product of this pseudoreal 4 with the complete set of irre- 
ducible representations of $Q_{6}$ is again real and that the resultant fermions are non-chiral.

The lesson is:

To obtain chiral fermions from compactification on $A d S_{5} \times$ $S_{5} / \Gamma$, the embedding of $\Gamma$ in $S U(4)$ must be such that the 4 of $S U(4)$ is neither real nor pseudoreal.

Other cases where the $\mathbf{4}$ of $S U(4)$ is complex, but not a complete irrep of $S U(2)$ coming from $Q_{6}$, and where the $\mathbf{6}$ of $S U(4)$ is real, are improper embeddings, but form perfectly good quiver gauge theories (not necessarily derivable from $A d S / C F T$ ). These theories may well be of phenomenological interest and are the analogs of the non-partition models related to the abelian orbifolded models. In the following we do not include these improper non-abelian orbifold model in our analysis, but one should keep them in mind as phenomenological viable gauge theories.

We will now enumerate all the possible minimal $N, \mathrm{~N}=0$ chiral models for non-abelian orbifolded $A d S_{5} \times S^{5}$ and then briefly consider breaking the resulting quiver gauge theory to the standard model. Our analysis concentrates on symmetry breaking via reduction to diagonal subgroups through either the Pati-Salam group $S U(4) \times S U(2) \times S U(2)$ or the trinification gauge group $S U(3) \times S U(3) \times S U(3)$ before reaching the standard model. While our list of proper ciral models is complete, we study only a limited number of possible patters on symmetry breaking, so our discussion should be used as a guide rather than a compendium. There are still many unexplored avenues for model building, both for abelian and non-abelian orbifolds. 
Chiral Fermions for all nonabelian $g \leq 31$

Looking at the full list of non-abelian discrete groups of order $g \leq 31$ as given explicitly in [194] we see that of the 45 such groups 32 are simple groups or semi-direct products thereof; these 32 are listed in the Table on page 4691 of [194], and reproduced in section 6.3 above. The remaining 13 are formed as semi-direct product groups (SDPGs) and are listed in the Table on page 4692 of [194] and in section 6.3. We shall follow closely this classification.

Using the pseudoreality considerations of the previous section, we can pare down the full list of 45 to only 19 which include 13 SDPGs. The lowest order nonabelian group $\Gamma$ which can lead to chiral fermions is $g=16$. The only possible orders $g \leq 31$ are the seven values: $g=16(5[5 S D P G s]), 18(2[1 S D P G])$, $20(1[1 S D P G]), \quad 21(1[1 S D P G]), \quad 24(6[3 S D P G s])$ $27(2[2 S D P G s])$, and $30(2[0 S D P G])$.

In parenthesis are the number of groups at order $g$ and the number of these that are SDPGS is in square brackets; they add to (19[13 SDPGs] ). We shall proceed with the analysis systematically, in progressively increasing magnitude of $g$. 


\section{4 $\mathcal{N}=0$ non-Abelian models \\ $\underline{\mathrm{g}=16 .}$}

The non-pseudoreal groups number five, and all are SDPGs. In the notation of Thomas and Wood [195], which we shall follow for definiteness, they are: 16/8, 9, 10,11,13. We now treat these in the order they are enumerated by Thomas and Wood. Again, the relevant multiplication tables are collected in Appendix A of [180].

Group 16/8; also designated $\left(Z_{4} \times Z_{2}\right) \tilde{\times} Z_{2}$.

This group has eight singlets $1_{1}, 1_{2}, \ldots \ldots, 1_{8}$ and two doublets $2_{1}$ and $2_{2}$. In the embedding of $16 / 8$ in $S U(4)$ we must avoid the singlet $1_{1}$ otherwise there will be a residual supersymmetry with $\mathcal{N} \geq 1$. Consider the embedding defined by $\boldsymbol{4}=\left(2_{1}, 2_{1}\right)$. To find the surviving chiral fermions we need to product the 4 with all ten of the irreps of $16 / 8$. This results in the table:

\begin{tabular}{||c||c|c|c|c|c|c|c|c||c|c||}
\hline & $1_{1}$ & $1_{2}$ & $1_{3}$ & $1_{4}$ & $1_{5}$ & $1_{6}$ & $1_{7}$ & $1_{8}$ & $2_{1}$ & $2_{2}$ \\
\hline \hline $1_{1}$ & & & & & & & & & $\times \times$ & \\
\hline $1_{2}$ & & & & & & & & & $\times \times$ & \\
\hline $1_{3}$ & & & & & & & & & $\times \times$ & \\
\hline $1_{4}$ & & & & & & & & & $\times \times$ & \\
\hline $1_{5}$ & & & & & & & & & & $\times \times$ \\
\hline $1_{6}$ & & & & & & & & & & $\times \times$ \\
\hline $1_{7}$ & & & & & & & & & & $\times \times$ \\
\hline $1_{8}$ & & & & & & & & & & $\times \times$ \\
\hline \hline $2_{1}$ & & & & & $\times \times$ & $\times \times$ & $\times \times$ & $\times \times$ & & \\
\hline $2_{2}$ & $\times \times$ & $\times \times$ & $\times \times$ & $\times \times$ & & & & & & \\
\hline \hline
\end{tabular}

If we choose $N=2$, the gauge group is $S U(2)^{8} \times S U(4)^{2}$, and the entries in the table correspond to bifundamental representations of this group (e.g., the entry nearest the top right corner at the position $\left(1_{1}, 2_{1}\right)$ is the representation $\left.2(2,1,1,1,1,1,1,1 ; \overline{4}, 1)\right)$. 
If we identify the diagonal subgroup of the first four $\mathrm{SU}(2) \mathrm{s}$ as $S U(2)_{L}$, of the second four as $S U(2)_{R}$ and of the two $\mathrm{SU}(4)$ as color $\mathrm{SU}(4)$ the result is non-chiral due to the lack of symmetry about the main diagonal of the above table.

On the other hand, if we identify $\mathbf{4}_{\mathbf{1}}$ with $\overline{\mathbf{4}}_{\mathbf{2}}$ there are potentially eight chiral families:

$$
8[(2,1,4)+(1,2, \overline{4})]
$$

under $S U(2)_{L} \times S U(2)_{R} \times S U(4)$. This is the maximum total chirality for this orbifold, but as we will see below, the allowed chiral at any stage is as usual determined by spontaneous symmetry breaking (SSB) generated in the scalar sector. In this section we give the maximum chirality for each orbifold, in the next section we study SSB for those models with sufficient chirality too accomodate at least three families.

Because $2_{1}=2_{2}^{*}$ form a complex conjugate pair, the embedding $\mathbf{4}=\left(2_{1}, 2_{2}\right)$ is pseudoreal $\mathbf{4} \equiv \mathbf{4}^{*}$ and the fermions are non-chiral as easily confirmed by the resultant table:

\begin{tabular}{||c||c|c|c|c|c|c|c|c||c|c||}
\hline & $1_{1}$ & $1_{2}$ & $1_{3}$ & $1_{4}$ & $1_{5}$ & $1_{6}$ & $1_{7}$ & $1_{8}$ & $2_{1}$ & $2_{2}$ \\
\hline \hline $1_{1}$ & & & & & & & & & $\times$ & $\times$ \\
\hline $1_{2}$ & & & & & & & & & $\times$ & $\times$ \\
\hline $1_{3}$ & & & & & & & & & $\times$ & $\times$ \\
\hline $1_{4}$ & & & & & & & & & $\times$ & $\times$ \\
\hline $1_{5}$ & & & & & & & & & $\times$ & $\times$ \\
\hline $1_{6}$ & & & & & & & & & $\times$ & $\times$ \\
\hline $1_{7}$ & & & & & & & & & $\times$ & $\times$ \\
\hline $1_{8}$ & & & & & & & & & $\times$ & $\times$ \\
\hline \hline $2_{1}$ & $\times$ & $\times$ & $\times$ & $\times$ & $\times$ & $\times$ & $\times$ & $\times$ & & \\
\hline $2_{2}$ & $\times$ & $\times$ & $\times$ & $\times$ & $\times$ & $\times$ & $\times$ & $\times$ & & \\
\hline \hline
\end{tabular}

For this embedding, the result is non-chiral for either of the cases $\mathbf{4}_{\mathbf{1}} \equiv \mathbf{4}_{\mathbf{2}}$ or $\mathbf{4}_{\mathbf{1}} \equiv \overline{\mathbf{4}}_{\mathbf{2}}$. (In the future, we shall not even consider such trivially real or pseudo-real non-chiral embeddings). 
Finally, for $16 / 8$, consider the embedding $4=\left(1_{2}, 1_{5}, 2_{1}\right)$. (In general there will be many equivalent embeddings. We will give one member of each equivalence class. Cases that are obviously nonchiral (vectorlike) will, in general, be ignored, except for a few instructive examples at orders 16 and 18.) The table is now:

\begin{tabular}{||c||c|c|c|c|c|c|c|c||c|c||}
\hline & $1_{1}$ & $1_{2}$ & $1_{3}$ & $1_{4}$ & $1_{5}$ & $1_{6}$ & $1_{7}$ & $1_{8}$ & $2_{1}$ & $2_{2}$ \\
\hline \hline $1_{1}$ & & $\times$ & & & $\times$ & & & & $\times$ & \\
\hline $1_{2}$ & $\times$ & & & & & $\times$ & & & $\times$ & \\
\hline $1_{3}$ & & & & $\times$ & & & $\times$ & & $\times$ & \\
\hline $1_{4}$ & & & $\times$ & & & & & $\times$ & $\times$ & \\
\hline $1_{5}$ & $\times$ & & & & & & $\times$ & & & $\times$ \\
\hline $1_{6}$ & & $\times$ & & & & $\times$ & & & & $\times$ \\
\hline $1_{7}$ & & & $\times$ & & & & & $\times$ & & $\times$ \\
\hline $1_{8}$ & & & & $\times$ & & & $\times$ & & & $\times$ \\
\hline \hline $2_{1}$ & & & & & $\times$ & $\times$ & $\times$ & $\times$ & $\times$ & $\times$ \\
\hline $2_{2}$ & $\times$ & $\times$ & $\times$ & $\times$ & & & & & $\times$ & $\times$ \\
\hline \hline
\end{tabular}

which is chiral.

These examples of embeddings for $\Gamma=16 / 8$ show clearly how the number of chiral families depends critically on the choice of embedding $\Gamma \subset S U(4)$. To actual achieve a model with a viable phenomenologically, we must study the possible routes through SSB for each chiral model. This we postpone until we have found all models of potential interest.

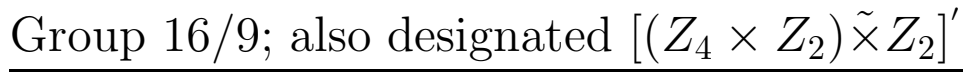

This group has irreps which comprise eight singlets $1_{1}, \ldots, 1_{8}$ and two doublets $2_{1}, 2_{2}$. With the embedding $\mathbf{4}=\left(2_{1}, 2_{2}\right)$ and using the multiplication table from Appendix A of [180] we arrive at the table of fermion bilinears: 


\begin{tabular}{||l||c|c|c|c|c|c|c|c||c|c||}
\hline & $1_{1}$ & $1_{2}$ & $1_{3}$ & $1_{4}$ & $1_{5}$ & $1_{6}$ & $1_{7}$ & $1_{8}$ & $2_{1}$ & $2_{2}$ \\
\hline \hline $1_{1}$ & & & & & & & & & $\times \times$ & \\
\hline $1_{2}$ & & & & & & & & & & $\times \times$ \\
\hline $1_{3}$ & & & & & & & & & $\times \times$ & \\
\hline $1_{4}$ & & & & & & & & & & $\times \times$ \\
\hline $1_{5}$ & & & & & & & & & $\times \times$ & \\
\hline $1_{6}$ & & & & & & & & & & $\times \times$ \\
\hline $1_{7}$ & & & & & & & & & $\times \times$ & \\
\hline $1_{8}$ & & & & & & & & & & $\times \times$ \\
\hline \hline $2_{1}$ & $\times \times$ & & $\times \times$ & & $\times \times$ & & $\times \times$ & & & \\
\hline $2_{2}$ & & $\times \times$ & & $\times \times$ & & $\times \times$ & & $\times \times$ & & \\
\hline \hline
\end{tabular}

This is non-chiral and has no families. This was the only potentially chiral embedding. In what follows, nonchiral models will not be displayed, however, as the unification scale can be rather low in AdS/CFT models, it would also be interesting to investigate vectorlike models of this class.

Group 16/10; also designated $Z_{4} \tilde{\times} Z_{4}$

The multiplication table is identical to that for $16 / 9$, as mentioned in Appendix A of [180]; thus the model building for 16/10 is also identical to $16 / 9$ and merits no additional discussion.

$\underline{\text { Group } 16 / 11 ; \text { also designated } Z_{8} \tilde{\times} Z_{2}}$

Again there are eight singlets and two doublets. The singlets $1_{1,3,5,7}$ are real while the other singlets fall into two conjugate pairs: $1_{2}=1_{4}^{*}$ and $1_{6}=1_{8}^{*}$. The doublets are complex: $2_{1}=2_{2}^{*}$.

The multiplication table includes the products: $1_{1,3,5,7} \times 2_{1,2}=$ $2_{1,2}$ and $1_{2,4,6,8} \times 2_{1,2}=2_{2,1}$. Also $2_{1} \times 2_{1}=2_{2} \times 2_{2}=1_{2}+1_{4}+$ $1_{6}+1_{8}$, while $2_{1} \times 2_{2}=1_{1}+1_{3}+1_{5}+1_{7}$.

This means that there are no interesting (legitimate and chiral) embeddings of the type $1+1+2$ or $2+2$. 
Upon choosing $N=3$, the most chiral possibility is the embedding $\mathbf{4}=\left(1_{2}, 1_{2}, 1_{2}, 1_{2}\right)$ which leads to the fermions in the following table. In this table, $(x)^{6} \equiv(x \times x \times x \times)$.

\begin{tabular}{||c||c|c|c|c|c|c|c|c||c|c||}
\hline & $1_{1}$ & $1_{2}$ & $1_{3}$ & $1_{4}$ & $1_{5}$ & $1_{6}$ & $1_{7}$ & $1_{8}$ & $2_{1}$ & $2_{2}$ \\
\hline \hline $1_{1}$ & & $(\times)^{6}$ & & & & & & & & \\
\hline $1_{2}$ & & & $(\times)^{6}$ & & & & & & & \\
\hline $1_{3}$ & & & & $(\times)^{6}$ & & & & & & \\
\hline $1_{4}$ & $(\times)^{6}$ & & & & & & & & & \\
\hline $1_{5}$ & & & & & & $(\times)^{6}$ & & & & \\
\hline $1_{6}$ & & & & & & & $(\times)^{6}$ & & & \\
\hline $1_{7}$ & & & & & & & & $(\times)^{6}$ & & \\
\hline $1_{8}$ & & & & & $(\times)^{6}$ & & & & & \\
\hline \hline $2_{1}$ & & & & & & & & & & $(\times)^{6}$ \\
\hline $2_{2}$ & & & & & & & & & $(\times)^{6}$ & \\
\hline \hline
\end{tabular}

This gives rise to twelve chiral families if we identify $N=3$ and $3_{1}=3_{4}=3_{5}=3_{8}, 3_{2}=3_{6}$ and $3_{3}=3_{7}$. Under $S U(3)^{3}$ the chiral fermions are:

$$
12[(3, \overline{3}, 1)+(1,3, \overline{3})+(\overline{3}, 3,1)]
$$

together with real non-chiral representations. When we discuss spontaneous symmetry breaking, we will see if this type of unification is possible.

With the different embedding $\boldsymbol{4}=\left(1_{2}, 1_{2}, 1_{2}, 1_{4}\right)$ the model changes to a less chiral but still interesting fermion configuration:

\begin{tabular}{||c||c|c|c|c|c|c|c|c||c|c||}
\hline & $1_{1}$ & $1_{2}$ & $1_{3}$ & $1_{4}$ & $1_{5}$ & $1_{6}$ & $1_{7}$ & $1_{8}$ & $2_{1}$ & $2_{2}$ \\
\hline \hline $1_{1}$ & $\times$ & $\times \times \times$ & & $\times$ & & & & & & \\
\hline $1_{2}$ & $\times$ & $\times$ & $\times \times \times$ & $\times \times \times$ & & & & & & \\
\hline $1_{4}$ & $\times \times \times$ & & $\times$ & & & & & & & \\
\hline $1_{5}$ & & & & & & $\times \times \times$ & & $\times$ & & \\
\hline $1_{6}$ & & & & & $\times$ & $\times$ & $\times \times \times$ & $\times \times \times$ & & \\
\hline $1_{7}$ & & & & & $\times \times \times$ & & $\times$ & & & \\
\hline \hline $2_{1}$ & & & & & & & & & & $\times \times \times \times$ \\
\hline $2_{2}$ & & & & & & & & & $\times \times \times \times$ & \\
\hline \hline
\end{tabular}

If we can identify $S U(3)^{\prime} s$ as $3_{1} \equiv 3_{4} \equiv 3_{5} \equiv 3_{8}, 3_{2} \equiv 3_{6}$ and $3_{3} \equiv 3_{7}$ this embedding give just four chiral families: 


$$
4[(3, \overline{3}, 1)+(1,3, \overline{3})+(\overline{3}, 3,1)]
$$

under $S U(3)^{3}$ together with real representations.

To check consistency, we have verified that real and legitimate embeddings for 16/11 like: $\boldsymbol{4}=\left(1_{3}, 1_{3}, 1_{3}, 1_{3}\right)$ and $\boldsymbol{4}=\left(2_{1}, 2_{2}\right)$ give no chiral fermions. 
$\underline{\text { Group } 16 / 13 ; \text { also designated }\left[Z_{8} \tilde{\times} Z_{2}\right]^{\prime \prime}}$

Of the five non-pseudoreal $g=16$ nonabelian $\Gamma, 16 / 13$ is unique in having only four inequivalent singlets $1_{1}, 1_{2}, 1_{3}, 1_{4}$ but three doublets $2_{1}, 2_{2}, 2_{3}$.

All the four singlet are real $1_{i}=1_{i}^{*}$. The three doublets comprise a conjugate complex pair $2_{1}=2_{3}^{*} \neq 2_{1}^{*}$ and the real $2_{2}=2_{2}^{*}$.

With the embedding $\mathbf{4}=\left(1_{3}, 1_{4}, 2_{1}\right)$ the resultant model has a chiral fermion quiver corresponding to the Table:

\begin{tabular}{||c||c|c|c|c||c|c|c||}
\hline & $1_{1}$ & $1_{2}$ & $1_{3}$ & $1_{4}$ & $2_{1}$ & $2_{2}$ & $2_{3}$ \\
\hline \hline $1_{1}$ & & & $\times$ & $\times$ & $\times$ & & \\
\hline $1_{2}$ & & & $\times$ & $\times$ & & & $\times$ \\
\hline $1_{3}$ & $\times$ & $\times$ & & & & & $\times$ \\
\hline $1_{4}$ & $\times$ & $\times$ & & & $\times$ & & \\
\hline \hline $2_{1}$ & & $\times$ & $\times$ & & $\times$ & $\times$ & $\times$ \\
\hline $2_{2}$ & & & & & $\times$ & $\times \times$ & $\times$ \\
\hline $2_{3}$ & $\times$ & & & $\times$ & $\times$ & $\times$ & $\times$ \\
\hline
\end{tabular}

Here and in the following, unless otherwise specified, we let $N=$ 2. If we identify $S U(2)_{L}$ with the diagonal subgroup of the first and fourth $S U(2)$ s, and $S U(2)_{R}$ with the diagonal subgroup of the 2nd and 3rd, then there are four chiral families if we embed $\boldsymbol{4}_{\mathbf{1}} \equiv \overline{\mathbf{4}}_{\mathbf{3}}$ and break $S U(4)_{2}$ completely.

Again consider $16 / 13$ but now with $\mathbf{4}=\left(2_{1}, 2_{1}\right)$. The table becomes: 


\begin{tabular}{||l||c|c|c|c||c|c|c||}
\hline & $1_{1}$ & $1_{2}$ & $1_{3}$ & $1_{4}$ & $2_{1}$ & $2_{2}$ & $2_{3}$ \\
\hline \hline $1_{1}$ & & & & & $\times \times$ & & \\
\hline $1_{2}$ & & & & & & & $\times \times$ \\
\hline $1_{3}$ & & & & & & & $\times \times$ \\
\hline $1_{4}$ & & & & & $\times \times$ & & \\
\hline \hline $2_{1}$ & & $\times \times$ & $\times \times$ & & & $\times \times$ & \\
\hline $2_{2}$ & & & & & $\times \times$ & & $\times \times$ \\
\hline $2_{3}$ & $\times \times$ & & & $\times \times$ & & $\times \times$ & \\
\hline
\end{tabular}

With $\mathbf{4}_{\mathbf{1}} \equiv \overline{\mathbf{4}}_{\mathbf{3}}$ there are eight chiral families.

A similar result occurs, of course, for $\boldsymbol{4}=\left(2_{3}, 2_{3}\right)$. But the embedding $\mathbf{4}=\left(2_{1}, 2_{3}\right)$ is non-chiral, leading to the symmetric fermion quiver/table:

\begin{tabular}{||c||c|c|c|c||c|c|c||}
\hline & $1_{1}$ & $1_{2}$ & $1_{3}$ & $1_{4}$ & $2_{1}$ & $2_{2}$ & $2_{3}$ \\
\hline \hline $1_{1}$ & & & & & $\times$ & & $\times$ \\
\hline $1_{2}$ & & & & & $\times$ & & $\times$ \\
\hline $1_{3}$ & & & & & $\times$ & & $\times$ \\
\hline $1_{4}$ & & & & & $\times$ & & $\times$ \\
\hline \hline $2_{1}$ & $\times$ & $\times$ & $\times$ & $\times$ & & $\times \times$ & \\
\hline $2_{2}$ & & & & & $\times \times$ & & $\times \times$ \\
\hline $2_{3}$ & $\times$ & $\times$ & $\times$ & $\times$ & & $\times \times$ & \\
\hline
\end{tabular}

This arrangement is manifestly non-chiral because of the symmetry of the table. Even though $2_{1}$ and $2_{3}$ are complex, $2_{1}=2_{3}^{*}$, so $4^{*}=\left(2_{1}, 2_{3}\right)^{*}=\left(2_{3}, 2_{1}\right)$. We can rotate this within $S U(4)$ to $4=\left(2_{1}, 2_{3}\right)$. Therefore, the 4 is pseudoreal and the fermions are vectorlike as expected.

The embedding $\mathbf{4}=\left(2_{2}, 2_{2}\right)$ in $16 / 13$ gives rise to the table: 


\begin{tabular}{||l||c|c|c|c||c|c|c||}
\hline & $1_{1}$ & $1_{2}$ & $1_{3}$ & $1_{4}$ & $2_{1}$ & $2_{2}$ & $2_{3}$ \\
\hline \hline $1_{1}$ & & & & & & $\times \times$ & \\
\hline $1_{2}$ & & & & & & $\times \times$ & \\
\hline $1_{3}$ & & & & & & $\times \times$ & \\
\hline $1_{4}$ & & & & & & $\times \times$ & \\
\hline \hline $2_{1}$ & & & & & $\times \times$ & & $\times \times$ \\
\hline $2_{2}$ & $\times \times$ & $\times \times$ & $\times \times$ & $\times \times$ & & & \\
\hline $2_{3}$ & & & & & $\times \times$ & & $\times \times$ \\
\hline
\end{tabular}

This embedding leads to no chirality and zero families. 
Finally, the embedding $4=\left(2_{1}, 2_{2}\right)$ of $16 / 13$ leads to the intermediate situation:

\begin{tabular}{||c||c|c|c|c||c|c|c||}
\hline & $1_{1}$ & $1_{2}$ & $1_{3}$ & $1_{4}$ & $2_{1}$ & $2_{2}$ & $2_{3}$ \\
\hline \hline $1_{1}$ & & & & & $\times$ & $\times$ & \\
\hline $1_{2}$ & & & & & & $\times$ & $\times$ \\
\hline $1_{3}$ & & & & & & $\times$ & $\times$ \\
\hline $1_{4}$ & & & & & $\times$ & $\times$ & \\
\hline \hline $2_{1}$ & & $\times$ & $\times$ & & $\times$ & $\times$ & $\times$ \\
\hline $2_{2}$ & $\times$ & $\times$ & $\times$ & $\times$ & $\times$ & & $\times$ \\
\hline $2_{3}$ & $\times$ & & & $\times$ & $\times$ & $\times$ & $\times$ \\
\hline
\end{tabular}

This give rise to four chiral families with the identification $\mathbf{4}_{\mathbf{1}} \equiv$ $\overline{4}_{3}$.

To summarize the "double doublet" embeddings $\mathbf{4}=\left(2_{i}, 2_{j}\right)$ of 16/13: for the equivalent embeddings $(i, j)=(1,1)$ or $(3$, $3)$, there are up to eight chiral families; for the other mutually equivalent cases $(\mathrm{i}, \mathrm{j})=(1,2),(3,2),(2,3)$, or $(2,1)$ there are up to four chiral families and finally for the pseudoreal cases (i, $\mathrm{j})=(1,3),(3,1)$ and the real case $(2,2)$ there are, because of the mathematical theorem (and as we have now easily verified by direct calculation) no chiral fermions. 


$$
\underline{\mathrm{g}=18 .}
$$

Here the non-pseudoreal groups number two, and one is an SDPG. In the notation of Thomas and Wood [195] they are: $18 / 3,5$. So we now treat these in the order they are enumerated by Thomas and Wood.

Group 18/3; also designated $D_{3} \times Z_{3}$

This group has irreps which fall into six singlets $1,1^{\prime}, 1 \alpha, 1^{\prime} \alpha, 1 \alpha^{2}, 1^{\prime} \alpha^{2}$ and three doublets $2,2 \alpha, 2 \alpha^{2}$. Using the $D_{3}$ multiplication table from appendix A of [180] the embedding $\mathbf{4}=\left(1 \alpha, 1^{\prime}, 2 \alpha\right)$ yields the table:

\begin{tabular}{||c||c|c|c||c|c|c||c|c|c||}
\hline & 1 & $1^{\prime}$ & 2 & $1 \alpha$ & $1^{\prime} \alpha$ & $2 \alpha$ & $1 \alpha^{2}$ & $1^{\prime} \alpha^{2}$ & $2 \alpha^{2}$ \\
\hline \hline 1 & & $\times$ & & $\times$ & & $\times$ & & & \\
\hline $1^{\prime}$ & $\times$ & & & & $\times$ & $\times$ & & & \\
\hline 2 & & & $\times$ & $\times$ & $\times$ & $\times \times$ & & & \\
\hline \hline $1 \alpha$ & & & & & $\times$ & & $\times$ & & $\times$ \\
\hline $1^{\prime} \alpha$ & & & & $\times$ & & & & $\times$ & $\times$ \\
\hline $2 \alpha$ & & & & & & $\times$ & $\times$ & $\times$ & $\times \times$ \\
\hline \hline $1 \alpha^{2}$ & $\times$ & & $\times$ & & & & & $\times$ & \\
\hline $1^{\prime} \alpha^{2}$ & & $\times$ & $\times$ & & & & $\times$ & & \\
\hline $2 \alpha^{2}$ & $\times$ & $\times$ & $\times \times$ & & & & & & $\times$ \\
\hline
\end{tabular}

Identifying $S U(2)_{L, R}$ with the diagonal subgroups of respectively $S U(2)_{3} \times S U(2)_{4}$ and $S U(2)_{5} \times S U(2)_{6}$ gives rise to two chiral families when it is assumed that $S U(2)_{1,2}$ and $S U(4)_{1,2}$ are broken. 
$\underline{\text { Group 18/5; also designated }\left(Z_{3} \times Z_{3}\right) \tilde{\times} Z_{2}}$

This group has two singlets $1,1^{\prime}$ and four doublets $2_{1}, 2_{2}, 2_{3}, 2_{4}$. Using the multiplication table from [180] we compute the models corresponding to the three inequivalent embeddings $\mathbf{4}=$ $\left(1^{\prime}, 1^{\prime}, 2_{1}\right), \boldsymbol{4}=\left(2_{1}, 2_{1}\right)$ and $\mathbf{4}=\left(2_{1}, 2_{2}\right)$.

For $\mathbf{4}=\left(1^{\prime}, 1^{\prime}, 2_{1}\right)$ the table is:

\begin{tabular}{||c||c|c||c|c|c|c||}
\hline & 1 & $1^{\prime}$ & $2_{1}$ & $2_{2}$ & $2_{3}$ & $2_{4}$ \\
\hline \hline 1 & & $\times \times$ & $\times$ & & & \\
\hline $1^{\prime}$ & $\times \times$ & & $\times$ & & & \\
\hline \hline $2_{1}$ & $\times$ & $\times$ & $\times \times \times$ & & & \\
\hline $2_{2}$ & & & & $\times \times$ & $\times$ & $\times$ \\
\hline $2_{3}$ & & & & $\times$ & $\times \times$ & $\times$ \\
\hline $2_{4}$ & & & & $\times$ & $\times$ & $\times \times$ \\
\hline
\end{tabular}

This model is manifestly non-chiral due to the symmetry of the table. 
For $\mathbf{4}=\left(2_{1}, 2_{1}\right)$ the table is:

\begin{tabular}{||c||c|c||c|c|c|c||}
\hline & 1 & $1^{\prime}$ & $2_{1}$ & $2_{2}$ & $2_{3}$ & $2_{4}$ \\
\hline \hline 1 & & & $\times \times$ & & & \\
\hline $1^{\prime}$ & & & $\times \times$ & & & \\
\hline \hline $2_{1}$ & $\times \times$ & $\times \times$ & $\times \times$ & & & \\
\hline $2_{2}$ & & & & & $\times \times$ & $\times \times$ \\
\hline $2_{3}$ & & & & $\times \times$ & & $\times \times$ \\
\hline $2_{4}$ & & & & $\times \times$ & $\times \times$ & \\
\hline
\end{tabular}

This model is also manifestly non-chiral due to the symmetry of the table.

For $\mathbf{4}=\left(2_{1}, 2_{2}\right)$ the table is:

\begin{tabular}{||c||c|c||c|c|c|c||}
\hline & 1 & $1^{\prime}$ & $2_{1}$ & $2_{2}$ & $2_{3}$ & $2_{4}$ \\
\hline \hline 1 & & & $\times$ & $\times$ & & \\
\hline $1^{\prime}$ & & & $\times$ & $\times$ & & \\
\hline \hline $2_{1}$ & $\times$ & $\times$ & $\times$ & & $\times$ & $\times$ \\
\hline $2_{2}$ & $\times$ & $\times$ & & $\times$ & $\times$ & $\times$ \\
\hline $2_{3}$ & & & $\times$ & $\times$ & & $\times \times$ \\
\hline $2_{4}$ & & & $\times$ & $\times$ & $\times \times$ & \\
\hline
\end{tabular}

Again, this model is manifestly non-chiral. 18/5 does not lend itself to chirality. This is easy to understand when one realizes that all of the irreducible representations of $18 / 5$ are individually either real or pseudoreal [195] making a complex embedding of 4 impossible. 


$$
\underline{\mathrm{g}=20 .}
$$

One non-pseudoreal group, an SDPG. In the notation of Thomas and Wood [195] it is $20 / 5$.

$\underline{\text { Group 20/5; also designated } Z_{5} \tilde{\times} Z_{4}}$

The group has four singlets $1_{1}, 1_{2}, 1_{3}, 1_{4}$ and a 4 . The singlets $1_{1}, 1_{3}$ are real and the other two form a complex conjugate pair $1_{2}=1_{4}^{*}$. The $\mathbf{6}$ which is the antisymmetric product $\mathbf{6}=(4 \times 4)_{a}$ must be real for a legitimate embedding. The two inequivalent choices, bearing in mind the multiplication table provided in the Appendix of [180] are $\boldsymbol{4}=\left(1_{2}, 1_{2}, 1_{2}, 1_{2}\right)$ and $\boldsymbol{4}=\left(1_{2}, 1_{2}, 1_{2}, 1_{4}\right)$.

The first $\mathbf{4}=\left(1_{2}, 1_{2}, 1_{2}, 1_{2}\right)$ yields the chiral fermions in the following table:

\begin{tabular}{||c||c|c|c|c||c||}
\hline & $1_{1}$ & $1_{2}$ & $1_{3}$ & $1_{4}$ & 4 \\
\hline \hline $1_{1}$ & & $\times \times \times \times$ & & & \\
\hline $1_{2}$ & & & $\times \times \times \times$ & & \\
\hline $1_{3}$ & & & & $\times \times \times \times$ & \\
\hline $1_{4}$ & $\times \times \times \times$ & & & & \\
\hline \hline 4 & & & & & $\times \times \times \times \times$ \\
\hline
\end{tabular}

Putting $N=3$ this embedding gives four chiral families when we identify $S U(3)_{3} \equiv S U(3)_{4}$ and drop real representations, giving:

$$
4[(3, \overline{3}, 1)+(1,3, \overline{3})+(\overline{3}, 1,3)]
$$

under $S U(3) \times S U(3) \times S U(3)$. The chiral sector of this $20 / 5$ nonabelian orbifold coincides with a $Z_{4}$ orbifold. 


\begin{tabular}{||c||c|c|c|c||c||}
\hline & $1_{1}$ & $1_{2}$ & $1_{3}$ & $1_{4}$ & 4 \\
\hline \hline $1_{1}$ & & $\times \times \times$ & & $\times$ & \\
\hline $1_{2}$ & $\times$ & & $\times \times \times$ & & \\
\hline $1_{3}$ & & $\times$ & & $\times \times \times$ & \\
\hline $1_{4}$ & $\times \times \times$ & & $\times$ & & \\
\hline \hline 4 & & & & & $\times \times \times \times \times$ \\
\hline
\end{tabular}

Identifying $S U(3)_{3} \equiv S U(3)_{4}$ as before for $N=3$ this is less chiral and gives rise to just two chiral families.

$$
4[(3, \overline{3}, 1)+(1,3, \overline{3})+(\overline{3}, 1,3)]
$$

under $S U(3) \times S U(3) \times S U(3)$. 


$$
\underline{\mathrm{g}=21 .}
$$

One non-pseudoreal group, an SDPG. In the notation of Thomas and Wood [195] it is: $21 / 2$.

Group $21 / 2 ;$ also designated $Z_{7} \tilde{\times} Z_{3}$

This group has irreps which comprise three singlets $1_{1}, 1_{2}, 1_{3}$ and two triplets $3_{1}, 3_{2}$. With the embedding $\mathbf{4}=\left(1_{2}, 3_{1}\right)$ (recall that $1_{1}$ must be avoided to obtain $\mathcal{N}=0$ ), the resultant fermions are given by:

\begin{tabular}{||c||c|c|c||c|c||}
\hline & $1_{1}$ & $1_{2}$ & $1_{3}$ & $3_{1}$ & $3_{2}$ \\
\hline \hline $1_{1}$ & & $\times$ & & $\times$ & \\
\hline $1_{2}$ & & & $\times$ & $\times$ & \\
\hline $1_{3}$ & $\times$ & & & $\times$ & \\
\hline \hline $3_{1}$ & & & & $\times \times$ & $\times \times$ \\
\hline $3_{2}$ & $\times$ & $\times$ & $\times$ & $\times$ & $\times \times$ \\
\hline
\end{tabular}

Putting $N=2$, the gauge group is $S U(2)^{3} \times S U(6)^{2}$. Clearly the model is chiral as seen in the asymmetry of the table. For example, put $S U(2)_{L} \equiv S U(2)_{1}, S U(2)_{R} \equiv S U(2)_{2}$, break $S U(2)_{3}$ entirely and break $S U(6)^{2}$ such that $\mathbf{6}_{\mathbf{1}} \rightarrow \mathbf{4}, \mathbf{6}_{\mathbf{2}} \rightarrow \overline{\mathbf{4}}$, to find two chiral families. 


$$
\underline{\mathrm{g}=24} .
$$

The non-pseudoreal groups number six, and three are SDPGs.

In the notation of Thomas and Wood [195] they are: 24/7, 8, 9, 13, 14, 15 .

We now treat these in the order they are enumerated by Thomas and Wood.

Group 24/7; also designated $D_{4} \times Z_{3}$

This has twelve singlets $1_{1} \alpha^{i}, 1_{2} \alpha^{i}, 1_{3} \alpha^{i}, 1_{4} \alpha^{i}(\mathrm{i}=0$ - 2) and three doublets $2 \alpha^{i}(\mathrm{i}=0-2)$; here $\alpha=\exp (i \pi / 3)$. The embedding $4=\left(1_{1} \alpha, 1_{2}, 2 \alpha\right)$ was studied in detail in [178] where it was shown how it can lead to precisely three chiral families in the standard model.

For completeness we include the table for the chiral fermions (it was presented in a different equivalent way in [178]):

\begin{tabular}{||c||c|c|c|c|c||c|c|c|c|c||c|c|c|c|c||}
\hline & $1_{1}$ & $1_{2}$ & $1_{3}$ & $1_{4}$ & 2 & $1_{1} \alpha$ & $1_{2} \alpha$ & $1_{3} \alpha$ & $1_{4} \alpha$ & $2 \alpha$ & $1_{1} \alpha^{2}$ & $1_{2} \alpha^{2}$ & $1_{3} \alpha^{2}$ & $1_{4} \alpha^{2}$ & $2 \alpha^{2}$ \\
\hline \hline $1_{1}$ & & $\times$ & & & & $\times$ & & & & $\times$ & & & & & \\
\hline $1_{2}$ & $\times$ & & & & & & $\times$ & & & $\times$ & & & & & \\
\hline $1_{3}$ & & & & $\times$ & & & & $\times$ & & $\times$ & & & & & \\
\hline $1_{4}$ & & & $\times$ & & & & & & $\times$ & $\times$ & & & & & \\
\hline 2 & & & & & $\times$ & $\times$ & $\times$ & $\times$ & $\times$ & $\times$ & & & & & \\
\hline \hline $1_{1} \alpha$ & & & & & & & $\times$ & & & & $\times$ & & & & $\times$ \\
\hline $1_{2} \alpha$ & & & & & & $\times$ & & & & & & $\times$ & & & $\times$ \\
\hline $1_{3} \alpha$ & & & & & & & & & $\times$ & & & & $\times$ & & $\times$ \\
\hline $1_{4} \alpha$ & & & & & & & & $\times$ & & & & & & $\times$ & $\times$ \\
\hline $2 \alpha$ & & & & & & & & & & $\times$ & $\times$ & $\times$ & $\times$ & $\times$ & $\times$ \\
\hline \hline $1_{1} \alpha^{2}$ & $\times$ & & & & $\times$ & & & & & & & $\times$ & & & \\
\hline $1_{2} \alpha^{2}$ & & $\times$ & & & $\times$ & & & & & & $\times$ & & & & \\
\hline $1_{3} \alpha^{2}$ & & & $\times$ & & $\times$ & & & & & & & & & $\times$ & \\
\hline $1_{4} \alpha^{2}$ & & & & $\times$ & $\times$ & & & & & & & & $\times$ & & \\
\hline $2 \alpha^{2}$ & $\times$ & $\times$ & $\times$ & $\times$ & $\times$ & & & & & & & & & & $\times$ \\
\hline \hline
\end{tabular}

By identifying $S U(4)$ with the diagonal subgroup of $S U(4)_{2,3}$, breaking $S U(4)_{1}$ to $S U(2)_{L}^{\prime} \times S U(2)_{R}^{\prime}$, then identifying $S U(2)_{L}$ with the diagonal subgroup of $S U(2)_{6,7,8}$ and $S U(2)_{L}^{\prime}$ and $S U(2)_{R}$ with the diagonal subgroup of $S U(2)_{10,11,12}$ and $S U(2)_{R}^{\prime}$ then leads to a three-family model as explained in [178]. 
It is convenient to represent the chiral fermions in a quiver diagram. This model is especially interesting because, uniquely among the large number of models examined in this study, the prescribed scalars are sufficient to break the gauge symmetry to that of the standard model.

Group 24/8; also designated $Q \times Z_{3}$

The multiplication tables of $D_{4}$ and $Q$ and hence the multiplication tables of $24 / 7$ and $24 / 8$ are identical. Model building for $24 / 8$ is therefore the same as $24 / 7$ and merits no additional discussion.

Group 24/9; also designated $D_{3} \times Z_{4}$

This group generates one of the richest sets of chiral model in the class of models discussed in this paper. The group has as irreps eight singlets $\left(1_{1} \alpha^{j}, 1_{2} \alpha^{j}\right)$ and four doublets $2 \alpha^{j}(\mathrm{j}=0,1$, $2,3)$, where $\alpha=\exp (i \pi / 4)$.

The embedding $\mathbf{4}=\left(1_{1} \alpha^{a_{1}}, 1_{2} \alpha^{a_{2}}, 2 \alpha^{a_{3}}\right)$ must satisfy $a_{1} \neq 0$ (for $\mathcal{N}=0)$ and $a_{1}+a_{2}=-2 a_{3}(\bmod 4)$ (to ensure reality of $\mathbf{6}=$ $\left.(4 \times 4)_{a}\right)$. There are several interesting possibilities including: $\left.\left.\left.\left.\left(1_{1} \alpha, 1_{2} \alpha, 2 \alpha\right)\right),\left(1_{1} \alpha, 1_{2} \alpha^{3}, 2 \alpha^{2}\right)\right),\left(1_{1} \alpha^{2}, 1_{2}, 2 \alpha^{3}\right)\right),\left(1_{1} \alpha^{2}, 1_{2}, 2 \alpha\right)\right)$, and $\left.\left(1_{1} \alpha^{2}, 1_{2} \alpha^{2}, 2\right)\right)$. The third and fourth cases are equivalent as can be seen by letting $\alpha$ go to $\alpha^{-1}$, and the last case has only real fermions since $\alpha^{2}=-1$.

The fermions for $\mathbf{4}=\left(\mathbf{1}_{\mathbf{1}} \alpha^{\mathbf{2}}, \mathbf{1}_{2} \alpha^{2}, \mathbf{2}\right)$ are vectorlike.

Moving on to $24 / 9$ with $\mathbf{4}=\left(\mathbf{1}_{\mathbf{1}} \alpha, \mathbf{1}_{\mathbf{2}} \alpha^{\mathbf{3}}, \mathbf{2} \alpha^{\mathbf{2}}\right)$ we find the fermions are chiral and fall into the irrep: 


\begin{tabular}{|c||c|c|c|c|c|c|c|c|c|c|c|c|}
\hline & $1_{1}$ & $1_{2}$ & 2 & $1_{1} \alpha$ & $1_{2} \alpha$ & $2 \alpha$ & $1_{1} \alpha^{2}$ & $1_{2} \alpha^{2}$ & $2 \alpha^{2}$ & $1_{1} \alpha^{3}$ & $1_{2} \alpha^{3}$ & $2 \alpha^{3}$ \\
\hline \hline $1_{1}$ & & & & $\times$ & & & & & $\times$ & & $\times$ & \\
\hline $1_{2}$ & & & & & $\times$ & & & & $\times$ & $\times$ & & \\
\hline 2 & & & & & & $\times$ & $\times$ & $\times$ & $\times$ & & & $\times$ \\
\hline \hline $1_{1} \alpha$ & & $\times$ & & & & & $\times$ & & & & & $\times$ \\
\hline $1_{2} \alpha$ & $\times$ & & & & & & & $\times$ & & & & $\times$ \\
\hline $2 \alpha$ & & & $\times$ & & & & & & $\times$ & $\times$ & $\times$ & $\times$ \\
\hline \hline $1_{1} \alpha^{2}$ & & & $\times$ & & $\times$ & & & & & $\times$ & & \\
\hline $1_{2} \alpha^{2}$ & & & $\times$ & $\times$ & & & & & & & $\times$ & \\
\hline $2 \alpha^{2}$ & $\times$ & $\times$ & $\times$ & & & $\times$ & & & & & & $\times$ \\
\hline \hline $1_{1} \alpha^{3}$ & $\times$ & & & & & $\times$ & & $\times$ & & & & \\
\hline $1_{1} \alpha^{3}$ & & $\times$ & & & & $\times$ & $\times$ & & & & & \\
\hline $2 \alpha^{3}$ & & & $\times$ & $\times$ & $\times$ & $\times$ & & & $\times$ & & & \\
\hline \hline
\end{tabular}

With the embedding $\mathbf{4}=\left(1_{1} \alpha, 1_{2} \alpha, 2 \alpha\right)$ the chiral fermions are:

\begin{tabular}{|c||c|c|c|c|c|c|c|c|c|c|c|c|}
\hline & $1_{1}$ & $1_{2}$ & 2 & $1_{1} \alpha$ & $1_{2} \alpha$ & $2 \alpha$ & $1_{1} \alpha^{2}$ & $1_{2} \alpha^{2}$ & $2 \alpha^{2}$ & $1_{1} \alpha^{3}$ & $1_{2} \alpha^{3}$ & $2 \alpha^{3}$ \\
\hline \hline $1_{1}$ & & & & $\times$ & $\times$ & $\times$ & & & & & & \\
\hline $1_{2}$ & & & & $\times$ & $\times$ & $\times$ & & & & & & \\
\hline 2 & & & & $\times$ & $\times$ & $\times \times \times$ & & & & & & \\
\hline \hline $1_{1} \alpha$ & & & & & & & $\times$ & $\times$ & $\times$ & & & \\
\hline $1_{2} \alpha$ & & & & & & & $\times$ & $\times$ & $\times$ & & & \\
\hline $2 \alpha$ & & & & & & & $\times$ & $\times$ & $\times \times \times$ & & & \\
\hline \hline $1_{1} \alpha^{2}$ & & & & & & & & & & $\times$ & $\times$ & $\times$ \\
\hline $1_{2} \alpha^{2}$ & & & & & & & & & & $\times$ & $\times$ & $\times$ \\
\hline $2 \alpha^{2}$ & & & & & & & & & & $\times$ & $\times$ & $\times \times \times$ \\
\hline \hline $1_{1} \alpha^{3}$ & $\times$ & $\times$ & $\times$ & & & & & & & & & \\
\hline $1_{2} \alpha^{3}$ & $\times$ & $\times$ & $\times$ & & & & & & & & & \\
\hline $2 \alpha^{3}$ & $\times$ & $\times$ & $\times \times \times$ & & & & & & & & & \\
\hline \hline
\end{tabular}

Identifying $S U(2)_{L}$ with the diagonal subgroup of $S U(2)_{1,2,3,4}$, $S U(2)_{R}$ with the diagonal subgroup of $S U(2)_{5,6,7,8}$ and the $\mathbf{4}$ of $S U(4)$ with the $\mathbf{4}$ of $S U(4)_{2,3}$ and the $\overline{\mathbf{4}}$ of $S U(4)_{1,4}$ leads to eight chiral families. 
Taking the embedding $4=\left(1_{1} \alpha^{2}, 1_{2}, 2 \alpha\right)$ gives as chiral fermions:

\begin{tabular}{||c||c|c|c||c|c|c||c|c|c||c|c|c||}
\hline & $1_{1}$ & $1_{2}$ & 2 & $1_{1} \alpha$ & $1_{2} \alpha$ & $2 \alpha$ & $1_{1} \alpha^{2}$ & $1_{2} \alpha^{2}$ & $2 \alpha^{2}$ & $1_{1} \alpha^{3}$ & $1_{2} \alpha^{3}$ & $2 \alpha^{3}$ \\
\hline \hline $1_{1}$ & & $\times$ & & & & $\times$ & $\times$ & & & & & \\
\hline $1_{2}$ & $\times$ & & & & & $\times$ & & $\times$ & & & & \\
\hline 2 & & & $\times$ & $\times$ & $\times$ & $\times$ & & & $\times$ & & & \\
\hline \hline $1_{1} \alpha$ & & & & & $\times$ & & & & $\times$ & $\times$ & & \\
\hline $1_{2} \alpha$ & & & & $\times$ & & & & & $\times$ & & $\times$ & \\
\hline $2 \alpha$ & & & & & & $\times$ & $\times$ & $\times$ & $\times$ & & & $\times$ \\
\hline \hline $1_{1} \alpha^{2}$ & $\times$ & & & & & & & $\times$ & & & & $\times$ \\
\hline $1_{2} \alpha^{2}$ & & $\times$ & & & & & $\times$ & & & & & $\times$ \\
\hline $2 \alpha^{2}$ & & & $\times$ & & & & & & $\times$ & $\times$ & $\times$ & $\times$ \\
\hline \hline $1_{1} \alpha^{3}$ & & & $\times$ & $\times$ & & & & & & & $\times$ & \\
\hline $1_{2} \alpha^{3}$ & & & $\times$ & & $\times$ & & & & & $\times$ & & \\
\hline $2 \alpha^{3}$ & $\times$ & $\times$ & $\times$ & & & $\times$ & & & & & & $\times$ \\
\hline
\end{tabular}

We identify $S U(2)_{L}, S U(2)_{R}$ with the diagonal subgroups of $S U(2)_{1,2}$ and $S U(2)_{3,4}$, respectively and break completely $S U(2)_{5,6,7,8}$. The generalized color embedding $\mathbf{4} \equiv \mathbf{4}_{\mathbf{1}} \equiv \mathbf{4}_{\mathbf{2}} \equiv \overline{\mathbf{4}}_{\mathbf{3}} \equiv \overline{\mathbf{4}}_{\mathbf{4}}$ leads to four chiral families. This can be reduced to three families by further symmetry breaking.

An even more interesting embedding for $24 / 9$ is to set $4=$ $(2 \alpha, 2 \alpha)$ which gives a real 6 as required (since $\alpha^{2}=-1$ is real). The table for fermions is:

\begin{tabular}{||c||c|c|c||c|c|c||c|c|c||c|c|c||}
\hline & $1_{1}$ & $1_{2}$ & 2 & $1_{1} \alpha$ & $1_{2} \alpha$ & $2 \alpha$ & $1_{1} \alpha^{2}$ & $1_{2} \alpha^{2}$ & $2 \alpha^{2}$ & $1_{1} \alpha^{3}$ & $1_{2} \alpha^{3}$ & $2 \alpha^{3}$ \\
\hline \hline $1_{1}$ & & & & & & $\times \times$ & & & & & & \\
\hline $1_{2}$ & & & & & & $\times \times$ & & & & & & \\
\hline 2 & & & & $\times \times$ & $\times \times$ & $\times \times$ & & & & & & \\
\hline \hline $1_{1} \alpha$ & & & & & & & & & $\times \times$ & & & \\
\hline $1_{2} \alpha$ & & & & & & & & & $\times \times$ & & & \\
\hline $2 \alpha$ & & & & & & & $\times \times$ & $\times \times$ & $\times \times$ & & & \\
\hline \hline $1_{1} \alpha^{2}$ & & & & & & & & & & & & $\times \times$ \\
\hline $1_{2} \alpha^{2}$ & & & & & & & & & & & & $\times \times$ \\
\hline $2 \alpha^{2}$ & & & & & & & & & & $\times \times$ & $\times \times$ & $\times \times$ \\
\hline \hline $1_{1} \alpha^{3}$ & & & $\times \times$ & & & & & & & & & \\
\hline $1_{2} \alpha^{3}$ & & & $\times \times$ & & & & & & & & & \\
\hline $2 \alpha^{3}$ & $\times \times$ & $\times \times$ & $\times \times$ & & & & & & & & & \\
\hline
\end{tabular}


Identifying $S U(2)_{L}$ with the diagonal subgroup of $S U(2)_{1,3,5,7}$, $S U(2)_{R}$ with the diagonal subgroup of $S U(2)_{2,4,6,8}$, breaking $S U(4)_{1,3}$ and keeping the unbroken $S U(4)$ which is the diagonal subgroup of $S U(4)_{2,4}$ gives rise to eight chiral families:

$$
8[(2,1, \overline{4})+(1,2,4)]
$$

The possibility of achieving the relevant symmetry breaking will be examined below. 
$\underline{\text { Group 24/13; also designated } Q \tilde{\times} Z_{3}}$

This group has three singlets $1_{1}, 1_{2}, 1_{3}$, three doublets $2_{1}, 2_{2}, 2_{3}$ and one triplet 3. For $N=2$ the gauge group is therefore $S U(2)^{3} \times S U(4)^{3} \times S U(6)$.

With the embedding $\mathbf{4}=\left(2_{1}, 2_{2}\right)$ the chiral fermions are:

\begin{tabular}{||c||c|c|c||c|c|c||c||}
\hline & $1_{1}$ & $1_{2}$ & $1_{3}$ & $2_{1}$ & $2_{2}$ & $2_{3}$ & 3 \\
\hline \hline $1_{1}$ & & & & $\times$ & $\times$ & & \\
\hline $1_{2}$ & & & & & $\times$ & $\times$ & \\
\hline $1_{3}$ & & & & $\times$ & & $\times$ & \\
\hline \hline $2_{1}$ & $\times$ & $\times$ & & & & & $\times \times$ \\
\hline $2_{2}$ & & $\times$ & $\times$ & & & & $\times \times$ \\
\hline $2_{3}$ & $\times$ & & $\times$ & & & & $\times \times$ \\
\hline \hline 3 & & & & $\times \times$ & $\times \times$ & $\times \times$ & \\
\hline
\end{tabular}

If we identify $S U(2)_{L} \equiv S U(2)_{3}, S U(2)_{R} \equiv S U(2)_{2}$, and break $S U(2)_{1}$ there are two chiral families for $\mathbf{4} \equiv \mathbf{4}_{\mathbf{1}} \equiv \overline{\mathbf{4}}_{\mathbf{2}} \equiv \overline{\mathbf{4}}_{\mathbf{3}}$. 
If, instead, we embed $\mathbf{4}=\left(2_{2}, 2_{3}\right)$ the fermions fall according to the following table:

\begin{tabular}{||c||c|c|c||c|c|c||c||}
\hline & $1_{1}$ & $1_{2}$ & $1_{3}$ & $2_{1}$ & $2_{2}$ & $2_{3}$ & 3 \\
\hline \hline $1_{1}$ & & & & & $\times$ & $\times$ & \\
\hline $1_{2}$ & & & & $\times$ & & $\times$ & \\
\hline $1_{3}$ & & & & $\times$ & $\times$ & & \\
\hline \hline $2_{1}$ & & $\times$ & $\times$ & & & & $\times \times$ \\
\hline $2_{2}$ & $\times$ & & $\times$ & & & & $\times \times$ \\
\hline $2_{3}$ & $\times$ & $\times$ & & & & & $\times \times$ \\
\hline \hline 3 & & & & $\times \times$ & $\times \times$ & $\times \times$ & \\
\hline
\end{tabular}

This model is manifestly non-chiral because of the total symmetry of the table. 
$\underline{\text { Group 24/14; also designated } Z_{8} \tilde{\times} Z_{3}}$

There are eight singlets and four doublets, with multiplication table as in [180]. With the embedding $\mathbf{4}=\left(2_{2}, 2_{4}\right)$ one arrives at the fermions:

\begin{tabular}{||c||c|c|c|c|c|c|c|c||c|c|c|c||}
\hline & $1_{1}$ & $1_{2}$ & $1_{3}$ & $1_{4}$ & $1_{5}$ & $1_{6}$ & $1_{7}$ & $1_{8}$ & $2_{1}$ & $2_{2}$ & $2_{3}$ & $2_{4}$ \\
\hline \hline $1_{1}$ & & & & & & & & & & $\times$ & & $\times$ \\
\hline $1_{2}$ & & & & & & & & & $\times$ & & $\times$ & \\
\hline $1_{3}$ & & & & & & & & & & $\times$ & & $\times$ \\
\hline $1_{4}$ & & & & & & & & & $\times$ & & $\times$ & \\
\hline $1_{5}$ & & & & & & & & & & $\times$ & & $\times$ \\
\hline $1_{6}$ & & & & & & & & & $\times$ & & $\times$ & \\
\hline $1_{7}$ & & & & & & & & & & $\times$ & & $\times$ \\
\hline $1_{8}$ & & & & & & & & & $\times$ & & $\times$ & \\
\hline \hline $2_{1}$ & & $\times$ & & $\times$ & & $\times$ & & $\times$ & & $\times$ & & $\times$ \\
\hline $2_{2}$ & $\times$ & & $\times$ & & $\times$ & & $\times$ & & $\times$ & & $\times$ & \\
\hline $2_{3}$ & & $\times$ & & $\times$ & & $\times$ & & $\times$ & & $\times$ & & $\times$ \\
\hline $2_{4}$ & $\times$ & & $\times$ & & $\times$ & & $\times$ & & $\times$ & & $\times$ & \\
\hline
\end{tabular}

This arrangement has zero families.

A chiral embedding is $\mathbf{4}=\left(2_{1}, 2_{2}\right)$ giving rise to the fermions: 


\begin{tabular}{||c||c|c|c|c|c|c|c|c||c|c|c|c||}
\hline & $1_{1}$ & $1_{2}$ & $1_{3}$ & $1_{4}$ & $1_{5}$ & $1_{6}$ & $1_{7}$ & $1_{8}$ & $2_{1}$ & $2_{2}$ & $2_{3}$ & $2_{4}$ \\
\hline \hline $1_{1}$ & & & & & & & & & $\times$ & $\times$ & & \\
\hline $1_{2}$ & & & & & & & & & & $\times$ & $\times$ & \\
\hline $1_{3}$ & & & & & & & & & & & $\times$ & $\times$ \\
\hline $1_{4}$ & & & & & & & & & $\times$ & & & $\times$ \\
\hline $1_{5}$ & & & & & & & & & $\times$ & $\times$ & & \\
\hline $1_{6}$ & & & & & & & & & & $\times$ & $\times$ & \\
\hline $1_{7}$ & & & & & & & & & & & $\times$ & $\times$ \\
\hline $1_{8}$ & & & & & & & & & $\times$ & & & $\times$ \\
\hline \hline $2_{1}$ & $\times$ & $\times$ & & & $\times$ & $\times$ & & & $\times$ & $\times$ & & \\
\hline $2_{2}$ & & $\times$ & $\times$ & & & $\times$ & $\times$ & & & $\times$ & $\times$ & \\
\hline $2_{3}$ & & & $\times$ & $\times$ & & & $\times$ & $\times$ & & & $\times$ & $\times$ \\
\hline $2_{4}$ & $\times$ & & & $\times$ & $\times$ & & & $\times$ & $\times$ & & & $\times$ \\
\hline
\end{tabular}

If we identify $S U(2)_{L}$ as the diagonal subgroup of $S U(2)_{1,2,5,6}$ and $S U(2)_{R}$ as the diagonal subgroup of $S U(2)_{3,4,7,8}$, then identify the $\mathbf{4}$ of $S U(4)$ with the $\mathbf{4}$ of $S U(4)_{2,3}$ and the $\overline{\mathbf{4}}$ of $S U(4)_{1,4}$, this model has eight chiral families under $S U(2)_{L} \times S U(2)_{R} \times$ $S U(4)$.

$\underline{\text { Group 24/15; also designated } D_{4} \tilde{\times} Z_{3}}$

The group 24/15 has nine inequivalent irreducible representations, four singlets and five doublets.

With the embedding $\mathbf{4}=\left(2_{3}, 2_{5}\right)$, the fermion table is: 


\begin{tabular}{||c||c|c|c|c||c|c|c|c|c||}
\hline & $1_{1}$ & $1_{2}$ & $1_{3}$ & $1_{4}$ & $2_{1}$ & $2_{2}$ & $2_{3}$ & $2_{4}$ & $2_{5}$ \\
\hline \hline $1_{1}$ & & & & & & & $\times$ & & $\times$ \\
\hline $1_{2}$ & & & & & & & & $\times$ & $\times$ \\
\hline $1_{3}$ & & & & & & & $\times$ & & $\times$ \\
\hline $1_{4}$ & & & & & & & & $\times$ & $\times$ \\
\hline \hline $2_{1}$ & & & & & & & $\times$ & $\times \times$ & $\times$ \\
\hline $2_{2}$ & & & & & & & $\times \times$ & $\times$ & $\times$ \\
\hline $2_{3}$ & & $\times$ & & $\times$ & $\times \times$ & $\times$ & & & \\
\hline $2_{4}$ & $\times$ & & $\times$ & & $\times$ & $\times \times$ & & & \\
\hline $2_{5}$ & $\times$ & $\times$ & $\times$ & $\times$ & $\times$ & $\times$ & & & \\
\hline
\end{tabular}

Identifying $S U(2)_{L} \equiv S U(2)_{1,3}$ and $S U(2)_{R} \equiv S U(2)_{2,4}$ gives rise to two chiral families. 
Another chiral embedding is $\mathbf{4}=\left(1_{2}, 1_{3}, 2_{3}\right)$ which gives the chiral fermions:

\begin{tabular}{||c||c|c|c|c||c|c|c|c|c||}
\hline & $1_{1}$ & $1_{2}$ & $1_{3}$ & $1_{4}$ & $2_{1}$ & $2_{2}$ & $2_{3}$ & $2_{4}$ & $2_{5}$ \\
\hline \hline $1_{1}$ & & $\times$ & $\times$ & & & & $\times$ & & \\
\hline $1_{2}$ & $\times$ & & & $\times$ & & & & $\times$ & \\
\hline $1_{3}$ & $\times$ & & & $\times$ & & & $\times$ & & \\
\hline $1_{4}$ & & $\times$ & $\times$ & & & & & $\times$ & \\
\hline \hline $2_{1}$ & & & & & $\times$ & $\times$ & & $\times$ & $\times$ \\
\hline $2_{2}$ & & & & & $\times$ & $\times$ & $\times$ & & $\times$ \\
\hline $2_{3}$ & & $\times$ & & $\times$ & $\times$ & & $\times$ & $\times$ & \\
\hline $2_{4}$ & $\times$ & & $\times$ & & & $\times$ & $\times$ & $\times$ & \\
\hline $2_{5}$ & & & & & $\times$ & $\times$ & & & $\times \times$ \\
\hline
\end{tabular}

Identifying $S U(2)_{L}$ with the diagonal subgroup of $1_{1}$ and $1_{3}$, $S U(2)_{R}$ with $1_{2}$ and $1_{4}$, and then identifying $2_{3}=4$ and $2_{4}=\overline{4}$ and finally breaking the other three $S U(4)$ 's gives rise to six chiral families. 
As an alternative $24 / 15$ model we can embed $4=\left(2_{3}, 2_{3}\right)$ and obtain:

\begin{tabular}{||l||c|c|c|c||c|c|c|c|c||}
\hline & $1_{1}$ & $1_{2}$ & $1_{3}$ & $1_{4}$ & $2_{1}$ & $2_{2}$ & $2_{3}$ & $2_{4}$ & $2_{5}$ \\
\hline \hline $1_{1}$ & & & & & & & $\times \times$ & & \\
\hline $1_{2}$ & & & & & & & & $\times \times$ & \\
\hline $1_{3}$ & & & & & & & $\times \times$ & & \\
\hline $1_{4}$ & & & & & & & & $\times \times$ & \\
\hline \hline $2_{1}$ & & & & & & & & $\times \times$ & $\times \times$ \\
\hline $2_{2}$ & & & & & & & $\times \times$ & & $\times \times$ \\
\hline $2_{3}$ & & $\times \times$ & & $\times \times$ & $\times \times$ & & & & \\
\hline $2_{4}$ & $\times \times$ & & $\times \times$ & & & $\times \times$ & & & \\
\hline $2_{5}$ & & & & & $\times \times$ & $\times \times$ & & & \\
\hline
\end{tabular}

With $S U(2)_{L}, S U(2)_{R}$ as diagonal subgroups of $S U(2)_{1} \times S U(2)_{3}$ and $S U(2)_{2} \times S U(2)_{4}$ respectively, and breaking completely $S U(4)_{4}$, this leads to four chiral families. 


$$
\underline{\mathrm{g}=27 .}
$$

The non-pseudoreal groups number two and both are SDPGs. In the notation of Thomas and Wood [195] they are: 27/4, 5. So we now treat these in the order they are enumerated by Thomas and Wood.

Group 27/4; also designated $Z_{9} \tilde{\times} Z_{3}$

$27 / 4$ has nine singlet $1_{1}, \ldots ., 1_{9}$ and two triplet $3_{1}, 3_{2}$ irreducible representations.

We may choose the embedding $4=\left(1_{2}, 3_{1}\right)$. The chiral fermions are:

\begin{tabular}{||c||c|c|c|c|c|c|c|c|c||c|c||}
\hline & $1_{1}$ & $1_{2}$ & $1_{3}$ & $1_{4}$ & $1_{5}$ & $1_{6}$ & $1_{7}$ & $1_{8}$ & $1_{9}$ & $3_{1}$ & $3_{2}$ \\
\hline \hline $1_{1}$ & & $\times$ & & & & & & & & $\times$ & \\
\hline $1_{2}$ & & & $\times$ & & & & & & & $\times$ & \\
\hline $1_{3}$ & $\times$ & & & & & & & & & $\times$ & \\
\hline $1_{4}$ & & & & & $\times$ & & & & & $\times$ & \\
\hline $1_{5}$ & & & & & & $\times$ & & & & $\times$ & \\
\hline $1_{6}$ & & & & $\times$ & & & & & & $\times$ & \\
\hline $1_{7}$ & & & & & & & & $\times$ & & $\times$ & \\
\hline $1_{8}$ & & & & & & & & & $\times$ & $\times$ & \\
\hline $1_{9}$ & & & & & & & $\times$ & & & $\times$ & \\
\hline \hline $3_{1}$ & & & & & & & & & & $\times$ & $\times \times \times$ \\
\hline $3_{2}$ & $\times$ & $\times$ & $\times$ & $\times$ & $\times$ & $\times$ & $\times$ & $\times$ & $\times$ & & $\times$ \\
\hline \hline
\end{tabular}

Putting $N=2$, the gauge group is $S U(2)^{9} \times S U(6)_{1} \times S U(6)_{2}$ and the chiral fermions are, from the above table:

$$
\left(\sum_{i=1}^{i=9} 2_{i}, \overline{6}_{1}\right)+\left(6_{1}, \overline{6}_{1}+3\left(\overline{6}_{2}\right)\right)+\left(6_{2}, \sum_{i=1}^{i=9} 2_{i}\right)+\left(6_{2}, \overline{6}_{2}\right)
$$

Though asymmetric in representations, this result is anomalyfree with respect to both $S U(6)_{1}$ and $S U(6)_{2}$. 


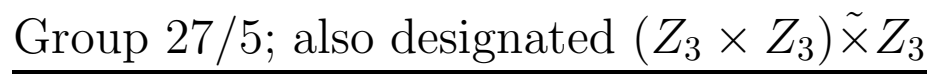

The multiplication tables, and hence the model-building, are identical for $27 / 4$ and $27 / 5$. The group $27 / 5$ merits no further separate discussion. 


$$
\underline{\mathrm{g}=30 .}
$$

The non-pseudoreal groups number two, and neither is an SDPG. In the notation of Thomas and Wood [195], they are: 30/2,3. We treat these in the order they are enumerated by Thomas and Wood.

Group 30/2; also designated $D_{5} \times Z_{3}$

$30 / 2$ has six singlets $1 \alpha^{i}, 1^{\prime} \alpha^{i}$ and six doublets $2 \alpha^{i}, 2^{\prime} \alpha^{i}$ with $\alpha=\exp (i \pi / 3)$ and $\mathrm{i}=0,1,2$.

Choosing $\mathbf{4}=\left(1 \alpha, 1^{\prime}, 2 \alpha\right)$ yields as fermions:

\begin{tabular}{||c||c|c|c|c||c|c|c|c||c|c|c|c||}
\hline & 1 & $1^{\prime}$ & 2 & $2^{\prime} \alpha$ & $1 \alpha$ & $1^{\prime} \alpha$ & $2 \alpha$ & $2^{\prime} \alpha$ & $1 \alpha^{2}$ & $1^{\prime} \alpha^{2}$ & $2 \alpha^{2}$ & $2^{\prime} \alpha^{2}$ \\
\hline \hline 1 & & $\times$ & & & $\times$ & & $\times$ & & & & & \\
\hline $1^{\prime}$ & $\times$ & & & & & $\times$ & $\times$ & & & & & \\
\hline 2 & & & $\times$ & & $\times$ & $\times$ & $\times$ & $\times$ & & & & \\
\hline $2^{\prime}$ & & & & $\times$ & & & $\times$ & $\times \times$ & & & & \\
\hline \hline $1 \alpha$ & & & & & & $\times$ & & & $\times$ & & $\times$ & \\
\hline $1^{\prime} \alpha$ & & & & & $\times$ & & & & & $\times$ & $\times$ & \\
\hline $2 \alpha$ & & & & & & & $\times$ & & $\times$ & $\times$ & $\times$ & $\times$ \\
\hline $2^{\prime} \alpha$ & & & & & & & & $\times$ & & & $\times$ & $\times \times$ \\
\hline \hline $1 \alpha^{2}$ & $\times$ & & $\times$ & & & & & & & $\times$ & & \\
\hline $1^{\prime} \alpha^{2}$ & & $\times$ & $\times$ & & & & & & $\times$ & & & \\
\hline $2 \alpha^{2}$ & $\times$ & $\times$ & $\times$ & $\times$ & & & & & & & $\times$ & \\
\hline $2^{\prime} \alpha^{2}$ & & & $\times$ & $\times \times$ & & & & & & & & $\times$ \\
\hline
\end{tabular}

Identify $S U(2)_{L}$ with the diagonal subgroup of $S U(2)_{1} \times S U(2)_{2}$ (associated with $1,1^{\prime}$ ) and $S U(2)_{R}$ with the diagonal subgroup of $S U(2)_{5} \times S U(2)_{6}$ (associated with $\left.1 \alpha^{2}, 1^{\prime} \alpha^{2}\right)$; break the $S U(4) \mathrm{s}$ associated with $2,2 \alpha^{2}$ to arrive at two chiral families. 
Group 30/3; also designated $D_{3} \times Z_{5}$

This group has irreps which comprise ten singlets and five doublets and yields, for $N=2$, the gauge group $S U(2)^{10} \times S U(4)^{5}$.

As we have encountered for groups $D_{3} \times Z_{p}$ (with $g=6 p$ ) the embedding $\mathbf{4}=\left(1 \alpha^{a_{1}}, 1^{\prime} \alpha^{a_{2}}, 2 \alpha^{a_{3}}\right)$ must satisfy $a_{1}+a_{2}=-2 a_{3}$ $(\bmod \mathrm{p})$ for consistency, as well as $a_{1} \neq 0$ to ensure $\mathcal{N}=0$.

There are several interesting such examples, one of which is $\mathbf{4}=$ $\left(1 \alpha, 1^{\prime}, 2 \alpha^{2}\right)$ which gives as fermions:

\begin{tabular}{||c||c|c|c||c|c|c||c|c|c||c|c|c||c|c|c|}
\hline & 1 & $1^{\prime}$ & 2 & $1 \alpha$ & $1^{\prime} \alpha$ & $2 \alpha$ & $1 \alpha^{2}$ & $1^{\prime} \alpha^{2}$ & $2 \alpha^{2}$ & $1 \alpha^{3}$ & $1^{\prime} \alpha^{3}$ & $2 \alpha^{3}$ & $1 \alpha^{4}$ & $1^{\prime} \alpha^{4}$ & $2 \alpha^{4}$ \\
\hline \hline 1 & & $\times$ & & $\times$ & & & & & $\times$ & & & & & & \\
\hline $1^{\prime}$ & $\times$ & & & & $\times$ & & & & $\times$ & & & & & & \\
\hline 2 & & & $\times$ & & & $\times$ & $\times$ & $\times$ & $\times$ & & & & & & \\
\hline \hline $1 \alpha$ & & & & & $\times$ & & $\times$ & & & & & $\times$ & & & \\
\hline $1^{\prime} \alpha$ & & & & $\times$ & & & & $\times$ & & & & $\times$ & & & \\
\hline $2 \alpha$ & & & & & & $\times$ & & & $\times$ & $\times$ & $\times$ & $\times$ & & & \\
\hline \hline $1 \alpha^{2}$ & & & & & & & & $\times$ & & $\times$ & & & & & $\times$ \\
\hline $1^{\prime} \alpha^{2}$ & & & & & & & $\times$ & & & & $\times$ & & & & $\times$ \\
\hline $2 \alpha^{2}$ & & & & & & & & & $\times$ & & & $\times$ & $\times$ & $\times$ & $\times$ \\
\hline \hline $1 \alpha^{3}$ & & & $\times$ & & & & & & & & $\times$ & & $\times$ & & \\
\hline $1^{\prime} \alpha^{3}$ & & & $\times$ & & & & & & & $\times$ & & & & $\times$ & \\
\hline $2 \alpha^{3}$ & $\times$ & $\times$ & $\times$ & & & & & & & & & $\times$ & & & $\times$ \\
\hline \hline $1 \alpha^{4}$ & $\times$ & & & & & $\times$ & & & & & & & & $\times$ & \\
\hline $1^{\prime} \alpha^{4}$ & & $\times$ & & & & $\times$ & & & & & & & $\times$ & & \\
\hline $2 \alpha^{4}$ & & & $\times$ & $\times$ & $\times$ & $\times$ & & & & & & & & & $\times$ \\
\hline
\end{tabular}

In an obvious notation, the chiral fermions are:

$$
\begin{aligned}
& \left(2_{1}+2_{2}, \overline{4}_{3}+4_{4}\right)+\left(2_{3}+2_{4}, \overline{4}_{4}+4_{5}\right)+\left(2_{5}+2_{6}, \overline{4}_{5}+4_{1}\right) \\
& +\left(2_{7}+2_{8}, \overline{4}_{1}+4_{2}\right)+\left(2_{9}+2_{10}, \overline{4}_{2}+4_{3}\right)
\end{aligned}
$$

By identifying, for example (there are equivalent cyclic permutations) $S U(2)_{L}$ as the diagonal subgroup of $S U(2)_{1} \times S U(2)_{2} \times$ $S U(2)_{7} \times S U(2)_{8}, S U(2)_{R}$ as the diagonal subgroup of $S U(2)_{5} \times$ 
$S U(2)_{6} \times S U(2)_{9} \times S U(2)_{10}$, generalized color $S U(4)$ as the diagonal subgroup of $S U(4)_{1} \times S U(4)_{3}$, and breaking completely $S U(4)_{2,4,5}$ give rise to four chiral families.

We can examine the infinite series $D_{3} \times Z_{p}$ for $p \geq 3$ (as necessary for non-pseudoreality). The order is $g=6 p$. By generalizing the above discussions of 18/3 $\left(D_{3} \times Z_{3}\right), 24 / 9\left(D_{3} \times Z_{4}\right)$ and $30 / 3$ $\left(D_{3} \times Z_{5}\right)$ we find that with the same type of embedding one arrives at a maximal number of $2[\mathrm{p}]$ chiral families where $[\mathrm{x}]$ is the largest integer not greater than $\mathrm{x}$. For example, with $\mathrm{p}=$ $3,4,5,6,7,8,9,10, \ldots$ one obtains $2,4,4,6,6,8,8,10 \ldots$ chiral families resspectively. This is an example of accessing the more difficult nonabelian $\Gamma$ with $g \geq 32$ at least for orders $g=6 p \geq 36$.

That completes the analysis of the occurrence of chiral fermions for $\Gamma$ with $g \leq 31$. For the cases where there are $\geq 3$ chiral families, it remains to check whether the spectrum of complex scalars is sufficient to allow spontaneous symmetry breaking to the Standard Model gauge group. 


\subsection{The Scalar Sector}

In order to carry out the spontaneous symmetry breaking (SSB) in the chiral models we found in the last section, we must first extract the scalar sector from eq. (5), where the 6 is gotten from the embedding of $(\mathbf{4} \times \mathbf{4})_{A}$ which in turn follows from the embedding of the $\mathbf{4}$. We only consider models of phenomenological interest, i.e., those which potentially have three or more families, but preferably three. With this perspective in mind we first collect the models, they are: 
$16 / 8$ with $\mathbf{4}=\left(\mathbf{2}_{1}, \mathbf{2}_{1}\right)$ and $\chi=2^{8}$ with $N=2$.

$16 / 8$ with $\mathbf{4}=\left(\mathbf{1}_{2}, \mathbf{1}_{5}, \mathbf{2}_{1}\right)$ and $\chi=2^{7}$ with $N=2$.

$\left.\overline{16 / 11 \text { with } \mathbf{4}=\left(\mathbf{1}_{2}, \mathbf{1}_{2}, \mathbf{1}_{2}\right.}, \mathbf{1}_{2}\right)$ and $\chi=432$ with $N=3$.

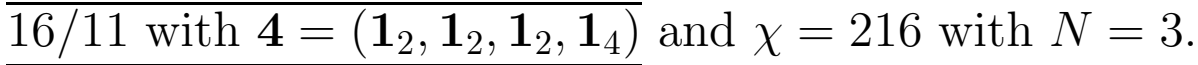

$16 / 13$ with $\mathbf{4}=\left(\mathbf{1}_{3}, \mathbf{1}_{4}, \mathbf{2}_{1}\right)$ and $\chi=2^{6}$ with $N=2$.

16/13 with $\mathbf{4}=\left(\mathbf{2}_{1}, \mathbf{2}_{2}\right)$ and $\chi=2^{6}$ with $N=2$

$\overline{16 / 13 \text { with } 4=\left(\mathbf{2}_{1}, \mathbf{2}_{1}\right)}$ and $\chi=2^{7}$ with $N=2$.

$18 / 3$ with $\mathbf{4}=\left(\mathbf{1} \alpha, \mathbf{1}^{\prime}, \mathbf{2} \alpha\right)$ and $\chi=192$ with $N=2$.

$20 / 5$ with $\mathbf{4}=\left(\mathbf{1}_{2}, \mathbf{1}_{2}, \mathbf{1}_{2}, \mathbf{1}_{2}\right)$ and $\chi=144$ with $N=3$.

$\overline{20 / 5 \text { with } \mathbf{4}=\left(\mathbf{1}_{2}, \mathbf{1}_{2}, \mathbf{1}_{2}, \mathbf{1}_{4}\right)}$ and $\chi=72$ with $N=3$.

$\overline{21 / 2 \text { with } \mathbf{4}=\left(\mathbf{1}_{2}, \mathbf{3}_{1}\right) \text { and } \chi}=108$ with $N=2$.

$24 / 7$ with $\mathbf{4}=\left(\mathbf{1} \alpha, \mathbf{1}^{\prime}, \mathbf{2} \alpha\right)$ and $\chi=240$ with $N=2$.

$24 / 9$ with $\mathbf{4}=\left(\mathbf{1}_{1} \alpha, \mathbf{1}_{2} \alpha^{3}, \mathbf{2} \alpha^{2}\right)$ and $\chi=320$ with $N=2$.

$24 / 9$ with $\mathbf{4}=\left(\mathbf{1}_{1} \alpha, \mathbf{1}_{2} \alpha, \mathbf{2} \alpha\right)$ and $\chi=320$ with $N=2$.

$24 / 9$ with $\mathbf{4}=\left(\mathbf{1}_{1} \alpha^{2}, \mathbf{1}_{2}, \mathbf{2} \alpha\right)$ and $\chi=192$ with $N=2$.

$\overline{24 / 9 \text { with } \mathbf{4}=(\mathbf{2} \alpha, \mathbf{2} \alpha) \text { and }} \chi=384$ with $N=2$.

$\overline{24 / 13 \text { with } \mathbf{4}=\left(\mathbf{2}_{1}, \mathbf{2}_{2}\right)}$ and $\chi=48$ with $N=2$.

$\overline{24 / 14 \text { with } \mathbf{4}=\left(\mathbf{2}_{1}, \mathbf{2}_{2}\right)}$ and $\chi=192$ with $N=2$.

$24 / 15$ with $\mathbf{4}=\left(\mathbf{1}_{2}, \mathbf{1}_{3}, \mathbf{2}_{3}\right)$ and $\chi=2^{7}$ with $N=2$.

24/15 with $4=\left(\mathbf{2}_{3}, \mathbf{2}_{5}\right)$ and $\chi=2^{7}$ with $N=2$.

24/15 with $4=\left(\mathbf{2}_{3}, \mathbf{2}_{3}\right)$ and $\chi=2^{8}$ with $N=2$.

$27 / 4$ with $\mathbf{4}=\left(\mathbf{1}_{2}, \mathbf{3}_{1}\right)$ and $\chi=324$ with $N=2$.

$30 / 2$ with $\mathbf{4}=\left(\mathbf{1} \alpha, \mathbf{1}^{\prime}, \mathbf{2} \alpha\right)$ and $\chi=336$ with $N=2$.

$\underline{30 / 3 \text { with } \mathbf{4}=\left(\mathbf{1} \alpha, \mathbf{1}^{\prime}, \mathbf{2} \alpha^{2}\right)}$ and $\chi=320$ with $N=2$.

where $\chi$ counts chirality, see below. 
First we consider $16 / 8$ with $\mathbf{4}=\left(\mathbf{1}_{2}, \mathbf{1}_{2}, \mathbf{2}_{1}\right)$, where we have included this example to demonstrate improper embedding. This representation is complex and would be expected to lead to chiral fermions, but $\mathbf{6}=(\mathbf{4} \times \mathbf{4})_{A}=\mathbf{1}_{1}+2\left(\mathbf{2}_{1}+\mathbf{2}_{1}\right)+\left(\mathbf{1}_{5}+\mathbf{1}_{6}+\mathbf{1}_{7}+\right.$ $\left.\mathbf{1}_{8}\right)_{A}$ is complex (for any choice of singlet in the last parenthetical expression), and therefore the embedding $\mathbf{4}=\left(\mathbf{1}_{2}, \mathbf{1}_{2}, \mathbf{2}_{1}\right)$ is improper and we need not consider this or other such models further.

Let us define the chirality measure $\chi$ of a model as the number of chiral fermion states. This variable applies to any irreps and provides a somewhat finer measure of chirality than the number of families. As spontaneous symmetry breaking (SSB) proceeds, $\chi$ decreased (except under unusual circumstances). For instance, the standard model and minimal $S U(5)$ both have $\chi=45$ initially. By the time the symmetry is broken to $S U(3) \times U_{E M}(1)$, $\chi=3$ since the neutrino's cannot acquire mass due to global $B-L$ symmetry. On the other hand, three family $S O(10)$ and $E_{6}$ models start with $\chi=48$ and $\chi=81$ respectively, but both break to $\chi=0$.

In model building with $A d S / C F T$ s we are faced with a number of choices. if we require the initial model be chiral before $\mathrm{SSB}$, then we need $\chi \geq 45$ initially. However, since the scale of SSB $M_{A d S}$ in these models can be relatively low (few 10s of $T e V$ ), vector like models are more appealing than usual, and we could allow an initial $\chi=0$ without resorting to incredibly detailed fine tunings. Our prejudice is to still require a chiral model with $\chi \geq 45$ initially in order to gain some control in model building, but we want to make it clear that, even though we have not displayed them explicitly, the entire class of vectorlike model based on the nonabelian orbifold classification given here would be worthy of detailed study. There are also models (chiral or vectorlike) that break from $G_{A d S}$ to $S U(3) \times U_{E M}(1)$ but without going through $S U(3) \times S U(2) \times U(1)$ directly. As 
$M_{A d S}$ may be not far above $M_{Z}$, there may be models in this class that could be in agreement with current data, but again we restrict most of our discussion to chiral models that break through the standard model. What is encouraging is the fact that orbifold AdS/CFTs provide such a wealth of potentially interesting models. 
We now begin a relatively systematic study of the scalar sectors of the chiral models.

$$
\underline{16 / 8 \text { with } \mathbf{4}=\left(\mathbf{2}_{1}, \mathbf{2}_{1}\right)} \text {. Here } \mathbf{6}=3\left(\mathbf{1}_{5}\right)+\mathbf{1}_{6}+\mathbf{1}_{7}+\mathbf{1}_{8} \text { which }
$$
is real so the embedding is proper and the scalar sector is:

\begin{tabular}{|c||c|c|c|c|c|c|c|c|c|c|}
\hline$\otimes$ & $1_{1}$ & $1_{2}$ & $1_{3}$ & $1_{4}$ & $1_{5}$ & $1_{6}$ & $1_{7}$ & $1_{8}$ & 2 & $2^{\prime}$ \\
\hline \hline $1_{1}$ & & & & & $\times \times \times$ & $\times$ & $\times$ & $\times$ & & \\
\hline $1_{2}$ & & & & & $\times$ & $\times$ & $\times$ & $\times$ & & \\
\hline $1_{3}$ & & & & & $\times$ & $\times$ & $\times$ & $\times$ & & \\
\hline $1_{4}$ & & & & & $\times$ & $\times$ & $\times$ & $\times \times \times$ & & \\
\hline $1_{5}$ & $\times \times \times$ & $\times$ & $\times$ & $\times$ & & & & & & \\
\hline $1_{6}$ & $\times$ & $\times$ & $\times$ & $\times$ & & & & & & \\
\hline $1_{7}$ & $\times$ & $\times$ & $\times$ & $\times$ & & & & & & \\
\hline $1_{8}$ & $\times$ & $\times$ & $\times$ & $\times \times \times$ & & & & & & \\
\hline 2 & & & & & & & & & & $\begin{array}{c}\times \times \times \\
\times \times \times\end{array}$ \\
\hline $2^{\prime}$ & & & & & & & & & $\begin{array}{c}\times \times \times \\
\times \times \times\end{array}$ & \\
\hline
\end{tabular}

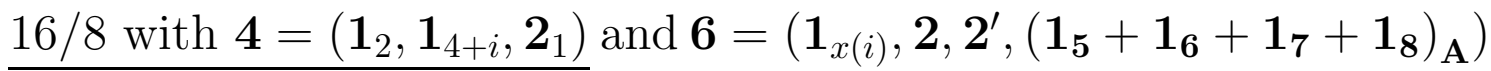
where $x=6,5,8$, or 7 for $i=1,2,3,4$. The fermionic sectors of these models are identical up to permutation, but there are two potential types of scalar sectors, depending on whether $\mathbf{1}_{x(i)}$ is the same as or different from the antisymmetric product $\left(\mathbf{2}_{1} \times \mathbf{2}_{1}\right)_{A}$. Let us relabel the singlets so $\left(\mathbf{2}_{1} \times \mathbf{2}_{1}\right)_{A}=\mathbf{1}_{\mathbf{6}}$, and then choose $\mathbf{1}_{x(i)}$ to be either $\mathbf{1}_{\mathbf{5}}$ or $\mathbf{1}_{\mathbf{6}}$. Now the two inequivalent scalar sectors (In this instance, it is easier to analyse both models and show that neither phenomenology is interesting, rather than untangle the correct antisymmetric singlet in $\left(\mathbf{2}_{1} \times \mathbf{2}_{1}\right)_{A}$. See the next section.) are: 


\begin{tabular}{|c||c|c|c|c|c|c|c|c|c|c|}
\hline$\otimes$ & $1_{1}$ & $1_{2}$ & $1_{3}$ & $1_{4}$ & $1_{5}$ & $1_{6}$ & $1_{7}$ & $1_{8}$ & 2 & $2^{\prime}$ \\
\hline \hline $1_{1}$ & & & & & $\times(5)$ & $(6)$ & & & $\times$ & $\times$ \\
\hline $1_{2}$ & & & & & $(6)$ & $\times(5)$ & & & $\times$ & $\times$ \\
\hline $1_{3}$ & & & & & & & $\times(5)$ & $(6)$ & $\times$ & $\times$ \\
\hline $1_{4}$ & & & & & & & $(6)$ & $\times(5)$ & $\times$ & $\times$ \\
\hline $1_{5}$ & $\times(5)$ & $(6)$ & & & & & & & $\times$ & $\times$ \\
\hline $1_{6}$ & $(6)$ & $\times(5)$ & & & & & & & $\times$ & $\times$ \\
\hline $1_{7}$ & & & $\times(5)$ & $(6)$ & & & & & $\times$ & $\times$ \\
\hline $1_{8}$ & & & $(6)$ & $\times(5)$ & & & & & $\times$ & $\times$ \\
\hline 2 & $\times$ & $\times$ & $\times$ & $\times$ & $\times$ & $\times$ & $\times$ & $\times$ & & $\times \times$ \\
\hline $2^{\prime}$ & $\times$ & $\times$ & $\times$ & $\times$ & $\times$ & $\times$ & $\times$ & $\times$ & $\times \times$ & \\
\hline
\end{tabular}

where(5) is replaced by an " $\times$ " and (6) by a blank if $\mathbf{1}_{x(i)}=\mathbf{1}_{\mathbf{5}}$ and vice versa if $\mathbf{1}_{x(i)}=\mathbf{1}_{\mathbf{6}}$. 
For $16 / 11$ with $\mathbf{4}=\left(\mathbf{1}_{2}, \mathbf{1}_{2}, \mathbf{1}_{2}, \mathbf{1}_{2}\right)$ and $\mathbf{6}=\left(\mathbf{1}_{3}, \mathbf{1}_{3}, \mathbf{1}_{3}, \mathbf{1}_{3}, \mathbf{1}_{3}, \mathbf{1}_{3}\right)$ the scalars are:

\begin{tabular}{|c||c|c|c|c|c|c|c|c|c|c|}
\hline$\otimes$ & $1_{1}$ & $1_{2}$ & $1_{3}$ & $1_{4}$ & $1_{5}$ & $1_{6}$ & $1_{7}$ & $1_{8}$ & 2 & $2^{\prime}$ \\
\hline \hline $1_{1}$ & & & $(\times)^{6}$ & & & & & & & \\
\hline $1_{2}$ & & $(\times)^{6}$ & & & & & & & & \\
\hline $1_{3}$ & $(\times)^{6}$ & & & & & & & & & \\
\hline $1_{4}$ & & & & $(\times)^{6}$ & & & & & & \\
\hline $1_{5}$ & & & & & & & $(\times)^{6}$ & & & \\
\hline $1_{6}$ & & & & & & $(\times)^{6}$ & & & & \\
\hline $1_{7}$ & & & & & $(\times)^{6}$ & & & & & \\
\hline $1_{8}$ & & & & & & & & $(\times)^{6}$ & & \\
\hline 2 & & & & & & & & & $(\times)^{6}$ & \\
\hline $2^{\prime}$ & & & & & & & & & & $(\times)^{6}$ \\
\hline
\end{tabular}


Similarly, for $16 / 11$ with $\mathbf{4}=\left(\mathbf{1}_{2}, \mathbf{1}_{2}, \mathbf{1}_{2}, \mathbf{1}_{4}\right)$ and $\mathbf{6}=\left(\mathbf{1}_{1}, \mathbf{1}_{1}, \mathbf{1}_{1}, \mathbf{1}_{3}, \mathbf{1}_{3}, \mathbf{1}_{3}\right)$ we find the scalars:

\begin{tabular}{|c||c|c|c|c|c|c|c|c|c|c|}
\hline$\otimes$ & $1_{1}$ & $1_{2}$ & $1_{3}$ & $1_{4}$ & $1_{5}$ & $1_{6}$ & $1_{7}$ & $1_{8}$ & 2 & $2^{\prime}$ \\
\hline \hline $1_{1}$ & $(\times)^{3}$ & & $(\times)^{3}$ & & & & & & & \\
\hline $1_{2}$ & & $(\times)^{6}$ & & & & & & & & \\
\hline $1_{3}$ & $(\times)^{3}$ & & $(\times)^{3}$ & & & & & & & \\
\hline $1_{4}$ & & & & $(\times)^{6}$ & & & & & & \\
\hline $1_{5}$ & & & & & $(\times)^{3}$ & & $(\times)^{3}$ & & & \\
\hline $1_{6}$ & & & & & & $(\times)^{6}$ & & & & \\
\hline $1_{7}$ & & & & & $(\times)^{3}$ & & $(\times)^{3}$ & & & \\
\hline $1_{8}$ & & & & & & & & $(\times)^{6}$ & & \\
\hline 2 & & & & & & & & & $(\times)^{6}$ & \\
\hline $2^{\prime}$ & & & & & & & & & & $(\times)^{6}$ \\
\hline
\end{tabular}

Next, $16 / 13$ with $\mathbf{4}=\left(\mathbf{1}_{\mathbf{3}}, \mathbf{1}_{\mathbf{4}}, \mathbf{2}_{1}\right)$ and $\mathbf{6}=\left(\mathbf{1}_{2}, \mathbf{1}_{c}, \mathbf{2}_{1}, \mathbf{2}_{3}\right)$, where

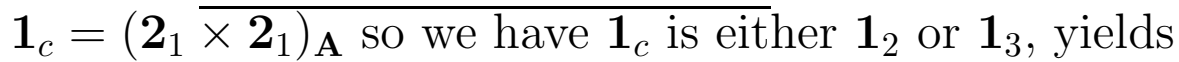

\begin{tabular}{|c||c|c|c|c|c|c|c|}
\hline$\otimes$ & $1_{1}$ & $1_{2}$ & $1_{3}$ & $1_{4}$ & $2_{1}$ & $2_{2}$ & $2_{3}$ \\
\hline \hline $1_{1}$ & & $\times(2)$ & $(3)$ & & $\times$ & & $\times$ \\
\hline $1_{2}$ & $\times(2)$ & & & $(3)$ & $\times$ & & $\times$ \\
\hline $1_{3}$ & $(3)$ & & & $\times(2)$ & $\times$ & & $\times$ \\
\hline $1_{4}$ & & $(3)$ & $\times(2)$ & & $\times$ & & $\times$ \\
\hline $2_{1}$ & $\times$ & $\times$ & $\times$ & $\times$ & & $\times \times$ & $\times \times$ \\
\hline $2_{2}$ & & & & & $\times \times$ & $\times \times$ & $\times \times$ \\
\hline $2_{3}$ & $\times$ & $\times$ & $\times$ & $\times$ & $\times \times$ & $\times \times$ & \\
\hline
\end{tabular}

Next, $16 / 13$ with $\mathbf{4}=\left(\mathbf{2}_{1}, \mathbf{2}_{2}\right)$ and $\mathbf{6}=\left(\mathbf{1}_{a}, \mathbf{1}_{b}, \mathbf{2}_{1}, \mathbf{2}_{3}\right)$, where $\mathbf{1}_{a}=\left(\mathbf{2}_{1} \overline{\left.\times \mathbf{2}_{1}\right)_{\mathbf{A}}=\left(1_{2}+1_{3}+2_{2}\right)_{\mathbf{A}}}\right.$ and $\mathbf{1}_{b}=\left(\mathbf{2}_{2} \times \mathbf{2}_{2}\right)_{\mathbf{A}}+\left(1_{1}+\right.$ $\left.1_{2}+1_{3}+1_{4}\right)_{\mathbf{A}}$ gives 


\begin{tabular}{|c||c|c|c|c|c|c|c|}
\hline$\otimes$ & $1_{1}$ & $1_{2}$ & $1_{3}$ & $1_{4}$ & $2_{1}$ & $2_{2}$ & $2_{3}$ \\
\hline \hline $1_{1}$ & $(1)$ & $(2)$ & $(3)$ & $(4)$ & $\times$ & & $\times$ \\
\hline $1_{2}$ & $(2)$ & $(1)$ & $(4)$ & $(3)$ & $\times$ & & $\times$ \\
\hline $1_{3}$ & $(3)$ & $(4)$ & $(1)$ & $(2)$ & $\times$ & & $\times$ \\
\hline $1_{4}$ & $(4)$ & $(3)$ & $(2)$ & $(1)$ & $\times$ & & $\times$ \\
\hline $2_{1}$ & $\times$ & $\times$ & $\times$ & $\times$ & $(1)(4)$ & $\times \times$ & $(2)(3)$ \\
\hline $2_{2}$ & & & & & $\times \times$ & $\begin{array}{c}(1)(2) \\
(3)(4)\end{array}$ & $\times \times$ \\
\hline $2_{3}$ & $\times$ & $\times$ & $\times$ & $\times$ & $(2)(3)$ & $\times \times$ & $(1)(4)$ \\
\hline
\end{tabular}

where again we insert $\times \mathrm{s}$ at the locations in parenthesis when the singlets are chosen properly from the antisymmetric products of the doublets. There are three inequivalent choices, either (i) put $\times \times$ at location (2), or (ii) put an $\times$ at (2) and one at (3), or (iii) put $\times$ at (2) and $\times$ at (1). All other choices lead to equivalent models. Thus, without the full detailed knowledge of the antisymmetric products, we can still reduce the analysis to the consideration of these three cases. 
Finally, we have $16 / 13$ with $\mathbf{4}=\left(\mathbf{2}_{1}, \mathbf{2}_{1}\right)$ and $\mathbf{6}=\left(\mathbf{1}_{2}, \mathbf{1}_{2}, \mathbf{1}_{2}, \mathbf{1}_{3}, \mathbf{2}_{2}\right)$ (which is equivalent to $\mathbf{6}=\left(\mathbf{1}_{2}, \mathbf{1}_{3}, \mathbf{1}_{3}, \mathbf{1}_{3}, \mathbf{2}_{2}\right)$ for SSB up to a relabeling of irreps).

\begin{tabular}{|c||c|c|c|c|c|c|c|}
\hline$\otimes$ & $1_{1}$ & $1_{2}$ & $1_{3}$ & $1_{4}$ & $2_{1}$ & $2_{2}$ & $2_{3}$ \\
\hline \hline $1_{1}$ & & $\times \times \times$ & $\times$ & & & $\times$ & \\
\hline $1_{2}$ & $\times \times \times$ & & & & & $\times$ & \\
\hline $1_{3}$ & $\times$ & & & $\times \times \times$ & & $\times$ & \\
\hline $1_{4}$ & & & $\times \times \times$ & & & $\times$ & \\
\hline $2_{1}$ & & & & & $\times$ & & $\begin{array}{c}\times \times \\
\times \times \times\end{array}$ \\
\hline $2_{2}$ & $\times$ & $\times$ & $\times$ & $\times$ & & $\begin{array}{c}\times \times \\
\times \times\end{array}$ & \\
\hline $2_{3}$ & & & & & $\begin{array}{l}\times \times \\
\times \times \times\end{array}$ & & $\times$ \\
\hline
\end{tabular}


Moving on to $o(\Gamma)=18$ we have $18 / 3$ with $4=\left(1^{\prime} \alpha, 1^{\prime}, 2 \alpha\right)$ and $6=\left(1^{\prime} \alpha, 2 \alpha, 2 \alpha^{2}, 1^{\prime} \alpha^{2}\right)$ with scalars

\begin{tabular}{|c||c|c|c|c|c|c|c|c|c|}
\hline$\otimes$ & 1 & $1^{\prime}$ & 2 & $1 \alpha$ & $1^{\prime} \alpha$ & $2 \alpha$ & $1 \alpha^{2}$ & $1^{\prime} \alpha^{2}$ & $2 \alpha^{2}$ \\
\hline \hline 1 & & & & & $\times$ & $\times$ & & $\times$ & $\times$ \\
\hline $1^{\prime}$ & & & & $\times$ & & $\times$ & $\times$ & & $\times$ \\
\hline 2 & & & & $\times$ & $\times$ & $\times \times$ & $\times$ & $\times$ & $\times \times$ \\
\hline $1 \alpha$ & & $\times$ & $\times$ & & & & & $\times$ & $\times$ \\
\hline $1^{\prime} \alpha$ & $\times$ & & $\times$ & & & & $\times$ & & $\times$ \\
\hline $2 \alpha$ & $\times$ & $\times$ & $\times \times$ & & & & $\times$ & $\times$ & $\times \times$ \\
\hline $1 \alpha^{2}$ & & $\times$ & $\times$ & & $\times$ & $\times$ & & & \\
\hline $1^{\prime} \alpha^{2}$ & $\times$ & & $\times$ & $\times$ & & $\times$ & & & \\
\hline $2 \alpha^{2}$ & $\times$ & $\times$ & $\times \times$ & $\times$ & $\times$ & $\times \times$ & & & \\
\hline
\end{tabular}


The two $o(\Gamma)=20$ models are $20 / 5$ with $\mathbf{4}=\left(\mathbf{1}_{2}, \mathbf{1}_{2}, \mathbf{1}_{2}, \mathbf{1}_{2}\right)$ and $\mathbf{6}=\left(\mathbf{1}_{3}, \mathbf{1}_{3}, \mathbf{1}_{3}, \mathbf{1}_{3}, \mathbf{1}_{3}, \mathbf{1}_{3}\right)$ which is very much like the $16 / 11$ model because of the similar embedding, with scalars

\begin{tabular}{|c||c|c|c|c|c|}
\hline$\otimes$ & $1_{1}$ & $1_{2}$ & $1_{3}$ & $1_{4}$ & 4 \\
\hline \hline $1_{1}$ & & & $(\times)^{6}$ & & \\
\hline $1_{2}$ & & & & $(\times)^{6}$ & \\
\hline $1_{3}$ & $(\times)^{6}$ & & & & \\
\hline $1_{4}$ & & $(\times)^{6}$ & & & \\
\hline 4 & & & & & \\
\hline
\end{tabular}

But a VEV for any of these renders the entire fermion sector vectorlike. 
The second model has $20 / 5$ with $\mathbf{4}=\left(\mathbf{1}_{2}, \mathbf{1}_{2}, \mathbf{1}_{2}, \mathbf{1}_{4}\right)$ and $\mathbf{6}=$ $\left(\mathbf{1}_{1}, \mathbf{1}_{1}, \mathbf{1}_{1}, \mathbf{1}_{3}, \mathbf{1}_{3}, \mathbf{1}_{3}\right)$ with scalars:

\begin{tabular}{|c||c|c|c|c|c|}
\hline$\otimes$ & $1_{1}$ & $1_{2}$ & $1_{3}$ & $1_{4}$ & 4 \\
\hline \hline $1_{1}$ & $\times \times \times$ & & $\times \times \times$ & & \\
\hline $1_{2}$ & & $\times \times \times$ & & $\times \times \times$ & \\
\hline $1_{3}$ & $\times \times \times$ & & $\times \times \times$ & & \\
\hline $1_{4}$ & & $\times \times \times$ & & $\times \times \times$ & \\
\hline 4 & & & & & $(\times)^{6}$ \\
\hline
\end{tabular}


At $o(\Gamma)=21$ we have a single model. $21 / 2$ with $\mathbf{4}=\left(\mathbf{1}_{2}, \mathbf{3}_{1}\right)$ and $\mathbf{6}=\mathbf{3}_{1}+\mathbf{3}_{2}$ with $N=2$. (All other embeddings of the 4 with chiral fermions and $\mathcal{N}=0$ SUSY are permutations and therefore equivalent to this model.). Here $\mathbf{6}$ is real so the embedding is proper and the scalar sector is:

\begin{tabular}{|c||c|c|c|c|c|}
\hline$\otimes$ & $1_{1}$ & $1_{2}$ & $1_{3}$ & $3_{1}$ & $3_{2}$ \\
\hline \hline $1_{1}$ & & & & $\times$ & $\times$ \\
\hline $1_{2}$ & & & & $\times$ & $\times$ \\
\hline $1_{3}$ & & & & $\times$ & $\times$ \\
\hline $3_{1}$ & $\times$ & $\times$ & $\times$ & $\times \times$ & $\times \times \times$ \\
\hline $3_{2}$ & $\times$ & $\times$ & $\times$ & $\times \times \times$ & $\times \times$ \\
\hline
\end{tabular}


The rich scalar sectors at $o(\Gamma)=24$ offer a number varied model building opportunities $24 / 7$ or equivalently $24 / 8$ (since they have isomorphic irrep product tables) with $4=$ $\left(\mathbf{1}_{1}, \alpha_{12}, \mathbf{2} \alpha\right)$ and $\mathbf{6}=\left(\mathbf{1}_{2} \alpha, \mathbf{1}_{2} \alpha^{2}, \mathbf{2} \alpha, \mathbf{2} \alpha^{2}\right)$ give the scalars

\begin{tabular}{|c||c|c|c|c|c|c|c|c|c|c|c|c|c|c|c|}
\hline$\otimes$ & $1_{1}$ & $1_{2}$ & $1_{3}$ & $1_{4}$ & 2 & $1_{1} \alpha$ & $1_{2} \alpha$ & $1_{3} \alpha$ & $1_{4} \alpha$ & $2 \alpha$ & $1_{1} \alpha^{2}$ & $1_{2} \alpha^{2}$ & $1_{3} \alpha^{2}$ & $1_{4} \alpha^{2}$ & $2 \alpha^{2}$ \\
\hline \hline $1_{1}$ & & & & & & & $\times$ & & & $\times$ & & $\times$ & & & $\times$ \\
\hline $1_{2}$ & & & & & & $\times$ & & & & $\times$ & $\times$ & & & & $\times$ \\
\hline $1_{3}$ & & & & & & & & & $\times$ & $\times$ & & & & $\times$ & $\times$ \\
\hline $1_{4}$ & & & & & & & & $\times$ & & $\times$ & & & $\times$ & & $\times$ \\
\hline 2 & & & & & & $\times$ & $\times$ & $\times$ & $\times$ & $\times$ & $\times$ & $\times$ & $\times$ & $\times$ & $\times$ \\
\hline $1_{1} \alpha$ & & $\times$ & & & $\times$ & & & & & & & $\times$ & & & $\times$ \\
\hline $1_{2} \alpha$ & $\times$ & & & & $\times$ & & & & & & $\times$ & & & & $\times$ \\
\hline $1_{3} \alpha$ & & & & $\times$ & $\times$ & & & & & & & & & $\times$ & $\times$ \\
\hline $1_{4} \alpha$ & & & $\times$ & & $\times$ & & & & & & & & $\times$ & & $\times$ \\
\hline $2 \alpha$ & $\times$ & $\times$ & $\times$ & $\times$ & $\times$ & & & & & & $\times$ & $\times$ & $\times$ & $\times$ & $\times$ \\
\hline $1_{1} \alpha^{2}$ & & $\times$ & & & $\times$ & & $\times$ & & & $\times$ & & & & & \\
\hline $1_{2} \alpha^{2}$ & $\times$ & & & & $\times$ & $\times$ & & & & $\times$ & & & & & \\
\hline $1_{3} \alpha^{2}$ & & & & $\times$ & $\times$ & & & & $\times$ & $\times$ & & & & & \\
\hline $1_{4} \alpha^{2}$ & & & $\times$ & & $\times$ & & & $\times$ & & $\times$ & & & & & \\
\hline $2 \alpha^{2}$ & $\times$ & $\times$ & $\times$ & $\times$ & $\times$ & $\times$ & $\times$ & $\times$ & $\times$ & $\times$ & & & & & \\
\hline
\end{tabular}


The scalars for $24 / 9$ with $\mathbf{4}=\left(\mathbf{1}_{\mathbf{1}} \alpha, \mathbf{1}_{\mathbf{2}} \alpha^{\mathbf{3}}, \mathbf{2} \alpha^{\mathbf{2}}\right)$ and $\mathbf{6}=\left(\mathbf{1}_{2}, \mathbf{1}_{2}, \mathbf{2} \alpha, \mathbf{2} \alpha^{3}\right)$ are:

\begin{tabular}{|c||c|c|c|c|c|c|c|c|c|c|c|c|}
\hline$\otimes$ & $1_{1}$ & $1_{2}$ & 2 & $1_{1} \alpha$ & $1_{2} \alpha$ & $2 \alpha$ & $1_{1} \alpha^{2}$ & $1_{2} \alpha^{2}$ & $2 \alpha^{2}$ & $1_{1} \alpha^{3}$ & $1_{2} \alpha^{3}$ & $2 \alpha^{3}$ \\
\hline \hline $1_{1}$ & & $\times \times$ & & & & $\times$ & & & & & & $\times$ \\
\hline $1_{2}$ & $\times \times$ & & & & & $\times$ & & & & & & $\times$ \\
\hline 2 & & & $\times \times$ & $\times$ & $\times$ & $\times$ & & & & $\times$ & $\times$ & $\times$ \\
\hline $1_{1} \alpha$ & & & $\times$ & & $\times \times$ & & & & $\times$ & & & \\
\hline $1_{2} \alpha$ & & & $\times$ & $\times \times$ & & & & & $\times$ & & & \\
\hline $2 \alpha$ & $\times$ & $\times$ & $\times$ & & & $\times \times$ & $\times$ & $\times$ & $\times$ & & & \\
\hline $1_{1} \alpha^{2}$ & & & & & & $\times$ & & $\times \times$ & & & & $\times$ \\
\hline $1_{2} \alpha^{2}$ & & & & & & $\times$ & $\times \times$ & & & & & $\times$ \\
\hline $2 \alpha^{2}$ & & & & $\times$ & $\times$ & $\times$ & & & $\times \times$ & $\times$ & $\times$ & $\times$ \\
\hline $1_{1} \alpha^{3}$ & & & $\times$ & & & & & & $\times$ & & $\times \times$ & \\
\hline $1_{1} \alpha^{3}$ & & & $\times$ & & & & & & $\times$ & $\times \times$ & & \\
\hline $2 \alpha^{3}$ & $\times$ & $\times$ & $\times$ & & & & $\times$ & $\times$ & $\times$ & & & $\times \times$ \\
\hline
\end{tabular}


The scalars for $24 / 9$ with $\mathbf{4}=\left(\mathbf{1}_{\mathbf{1}} \alpha, \mathbf{1}_{\mathbf{2}} \alpha, \mathbf{2} \alpha\right)$ and $\mathbf{6}=\left(\mathbf{1}_{\mathbf{2}} \alpha, \mathbf{2}, \mathbf{1}_{\mathbf{2}} \alpha, \mathbf{2} \alpha^{\mathbf{2}}\right)$ are:

\begin{tabular}{|c||c|c|c|c|c|c|c|c|c|c|c|c|}
\hline$\otimes$ & $1_{1}$ & $1_{2}$ & 2 & $1_{1} \alpha$ & $1_{2} \alpha$ & $2 \alpha$ & $1_{1} \alpha^{2}$ & $1_{2} \alpha^{2}$ & $2 \alpha^{2}$ & $1_{1} \alpha^{3}$ & $1_{2} \alpha^{3}$ & $2 \alpha^{3}$ \\
\hline \hline $1_{1}$ & & & & & & & & $\times \times$ & $\times \times$ & & & \\
\hline $1_{2}$ & & & & & & & $\times \times$ & & $\times \times$ & & & \\
\hline 2 & & & & & & & $\times \times$ & $\times \times$ & $\begin{array}{c}\times \times \\
\times \times\end{array}$ & & & \\
\hline $1_{1} \alpha$ & & & & & & & & & & & $\times \times$ & $\times \times$ \\
\hline $1_{2} \alpha$ & & & & & & & & & & $\times \times$ & & $\times \times$ \\
\hline $2 \alpha$ & & & & & & & & & & $\times \times$ & $\times \times$ & $\begin{array}{c}\times \\
\times\end{array}$ \\
\hline $1_{1} \alpha^{2}$ & & $\times \times$ & $\times \times$ & & & & & & & & & \\
\hline $1_{2} \alpha^{2}$ & $\times \times$ & & $\times \times$ & & & & & & & & & \\
\hline $2 \alpha^{2}$ & $\times \times$ & $\times \times$ & $\begin{array}{l}\times \times \\
\times \times\end{array}$ & & & & & & & & & \\
\hline $1_{1} \alpha^{3}$ & & & & & $\times \times$ & $\times \times$ & & & & & & \\
\hline $1_{1} \alpha^{3}$ & & & & $\times \times$ & & $\times \times$ & & & & & & \\
\hline $2 \alpha^{3}$ & & & & $\times \times$ & $\times \times$ & $\begin{array}{c}\times \times \\
\times \times\end{array}$ & & & & & & \\
\hline
\end{tabular}


For $24 / 9$ with $\mathbf{4}=\left(\mathbf{1}_{1} \alpha, \mathbf{1}_{2}, \mathbf{2} \alpha\right)$ and $\mathbf{6}=\left(\mathbf{1}_{2} \alpha^{2}, \mathbf{2} \alpha, \mathbf{2} \alpha^{-1}, \mathbf{1}_{2} \alpha^{-2}\right)$ where $\alpha^{4}=1$, the scalar sector is:

\begin{tabular}{|c||c|c|c|c|c|c|c|c|c|c|c|c|}
\hline$\otimes$ & $1_{1}$ & $1_{2}$ & 2 & $1_{1} \alpha$ & $1_{2} \alpha$ & $2 \alpha$ & $1_{1} \alpha^{2}$ & $1_{2} \alpha^{2}$ & $2 \alpha^{2}$ & $1_{1} \alpha^{3}$ & $1_{2} \alpha^{3}$ & $2 \alpha^{3}$ \\
\hline \hline $1_{1}$ & & & & & & $\times$ & & $\times \times$ & & & & $\times$ \\
\hline $1_{2}$ & & & & & & $\times$ & $\times \times$ & & & & & $\times$ \\
\hline 2 & & & & $\times$ & $\times$ & $\times$ & & & $\times \times$ & $\times$ & $\times$ & $\times$ \\
\hline $1_{1} \alpha$ & & & $\times$ & & & & & & $\times$ & & $\times \times$ & \\
\hline $1_{2} \alpha$ & & & $\times$ & & & & & & $\times$ & $\times \times$ & & \\
\hline $2 \alpha$ & $\times$ & $\times$ & $\times$ & & & & $\times$ & $\times$ & $\times$ & & & $\times \times$ \\
\hline $1_{1} \alpha^{2}$ & & $\times \times$ & & & & $\times$ & & & & & & $\times$ \\
\hline $1_{2} \alpha^{2}$ & $\times \times$ & & & & & $\times$ & & & & & & $\times$ \\
\hline $2 \alpha^{2}$ & & & $\times \times$ & $\times$ & $\times$ & $\times$ & & & & $\times$ & $\times$ & $\times$ \\
\hline $1_{1} \alpha^{3}$ & & & $\times$ & & $\times \times$ & & & & $\times$ & & & \\
\hline $1_{2} \alpha^{3}$ & & & $\times$ & $\times \times$ & & & & & $\times$ & & & \\
\hline $2 \alpha^{3}$ & $\times$ & $\times$ & $\times$ & & & $\times \times$ & $\times$ & $\times$ & $\times$ & & & \\
\hline
\end{tabular}


For $24 / 9$ with $\mathbf{4}=(\mathbf{2} \alpha, \mathbf{2} \alpha)$ where $\mathbf{6}=3\left(\mathbf{1}_{2} \alpha^{2}\right)+\mathbf{1}_{1} \alpha^{2}+$ $2 \alpha^{2}$, the scalars are:

\begin{tabular}{|c|c|c|c|c|c|c|c|c|c|c|c|c|}
\hline$\otimes$ & $1_{1}$ & $1_{2}$ & 2 & $1_{1} \alpha$ & $1_{2} \alpha$ & $2 \alpha$ & $1_{1} \alpha^{2}$ & $1_{2} \alpha^{2}$ & $2 \alpha^{2}$ & $1_{1} \alpha^{3}$ & $1_{2} \alpha^{3}$ & $2 \alpha^{3}$ \\
\hline $1_{1}$ & & & & & & & $x$ & $\times \times \times$ & $\times \times$ & & & \\
\hline $1_{2}$ & & & & & & & $\times \times \times$ & $\times$ & $\times \times$ & & & \\
\hline 2 & & & & & & & $\times \times$ & $\times \times$ & $\begin{array}{l}\times \times \times \\
\times \times \times\end{array}$ & & & \\
\hline $1_{1} \alpha$ & & & & & & & & & & $x$ & $\times \times \times$ & $\times x$ \\
\hline $1_{2} \alpha$ & & & & & & & & & & $\times \times \times$ & $\times$ & $\times \times$ \\
\hline $2 \alpha$ & & & & & & & & & & $\times \times$ & $\times \times$ & $\begin{array}{l}\times \times \times \\
\times \times \times\end{array}$ \\
\hline $1_{1} \alpha^{2}$ & $\times$ & $\times \times \times$ & $\times x$ & & & & & & & & & \\
\hline $1_{2} \alpha^{2}$ & $\times \times \times$ & $\times$ & $\times \times$ & & & & & & & & & \\
\hline $2 \alpha^{2}$ & $\times \times$ & $\times \times$ & $\begin{array}{l}\times \times \times \\
\times \times \times\end{array}$ & & & & & & & & & \\
\hline $1_{1} \alpha^{3}$ & & & & $\times$ & $\times \times \times$ & $\times \times$ & & & & & & \\
\hline $1_{2} \alpha^{3}$ & & & & $\times \times \times$ & $\times$ & $\times \times$ & & & & & & \\
\hline $2 \alpha^{3}$ & & & & $\times \times$ & $\times \times$ & $\begin{array}{l}\times \times \times \\
\times \times \times\end{array}$ & & & & & & \\
\hline
\end{tabular}


The next example of interest is $24 / 13$ with $\mathbf{4}=\left(\mathbf{2}_{1}, \mathbf{2}_{2}\right)$ and $\mathbf{6}=\mathbf{1}_{1}+\mathbf{1}_{2}+\mathbf{1}_{3}+\mathbf{3}$ with scalars:

\begin{tabular}{|c||c|c|c|c|c|c|c|}
\hline$\otimes$ & $1_{1}$ & $1_{2}$ & $1_{3}$ & $2_{1}$ & $2_{2}$ & $2_{3}$ & 3 \\
\hline \hline $1_{1}$ & $\times$ & $\times$ & $\times$ & & & & $\times$ \\
\hline $1_{2}$ & $\times$ & $\times$ & $\times$ & & & & $\times$ \\
\hline $1_{3}$ & $\times$ & $\times$ & $\times$ & & & & $\times$ \\
\hline $2_{1}$ & & & & $\times \times$ & $\times \times$ & $\times \times$ & \\
\hline $2_{2}$ & & & & $\times \times$ & $\times \times$ & $\times \times$ & \\
\hline $2_{3}$ & & & & $\times \times$ & $\times \times$ & $\times \times$ & \\
\hline 3 & $\times$ & $\times$ & $\times$ & & & & $\times \times$ \\
\hline
\end{tabular}

There are two ineqivalent models for the group 24/15, they are $\underline{\mathbf{4}=\left(\mathbf{1}_{2}, \mathbf{1}_{3}, \mathbf{2}_{3}\right)}$ where $\mathbf{6}=\mathbf{1}_{4}+\mathbf{1}_{2[4]}+\mathbf{2}_{3}+\mathbf{2}_{4}$ and the scalars are:

\begin{tabular}{|c||c|c|c|c|c|c|c|c|c|}
\hline$\otimes$ & $1_{1}$ & $1_{2}$ & $1_{3}$ & $1_{4}$ & $2_{1}$ & $2_{2}$ & $2_{3}$ & $2_{4}$ & $2_{5}$ \\
\hline \hline $1_{1}$ & & & & $\times \times$ & & & $\times$ & $\times$ & \\
\hline $1_{2}$ & & & $\times \times$ & & & & $\times$ & $\times$ & \\
\hline $1_{3}$ & & $\times \times$ & & & & & $\times$ & $\times$ & \\
\hline $1_{4}$ & $\times \times$ & & & & & & $\times$ & $\times$ & \\
\hline $2_{1}$ & & & & & & $\times \times$ & $\times$ & $\times$ & $\times \times$ \\
\hline $2_{2}$ & & & & & $\times \times$ & & $\times$ & $\times$ & $\times \times$ \\
\hline $2_{3}$ & $\times$ & $\times$ & $\times$ & $\times$ & $\times$ & $\times$ & & $\times \times$ & \\
\hline $2_{4}$ & $\times$ & $\times$ & $\times$ & $\times$ & $\times$ & $\times$ & $\times \times$ & & \\
\hline $2_{5}$ & & & & & $\times \times$ & $\times \times$ & & & $\times \times$ \\
\hline
\end{tabular}

if $\left(\mathbf{2}_{3} \times \mathbf{2}_{3}\right)_{A}=\mathbf{1}_{4}$ but if it is $\mathbf{1}_{2}$ then the top $4 \times 4$ changes to:

\begin{tabular}{||l|l|l|l|}
\hline \hline & $\times$ & & $\times$ \\
\hline$\times$ & & $\times$ & \\
\hline & $\times$ & & $\times$ \\
\hline$\times$ & & $\times$ & \\
\hline
\end{tabular}


The other $24 / 15$ case has $\mathbf{4}=\left(\mathbf{2}_{3}, \mathbf{2}_{3}\right)$ where $\mathbf{6}=3\left(\mathbf{1}_{2}\right)+$ $\mathbf{1}_{4}+\mathbf{2}_{1}$ and the scalars are (this time swapping $\mathbf{1}_{2}$ and $\mathbf{1}_{4}$ gives equivalent models):

\begin{tabular}{|c||c|c|c|c|c|c|c|c|c|}
\hline$\otimes$ & $1_{1}$ & $1_{2}$ & $1_{3}$ & $1_{4}$ & $2_{1}$ & $2_{2}$ & $2_{3}$ & $2_{4}$ & $2_{5}$ \\
\hline \hline $1_{1}$ & & $\times \times \times$ & & $\times$ & $\times$ & & & & \\
\hline $1_{2}$ & $\times \times \times$ & & $\times$ & & & $\times$ & & & \\
\hline $1_{3}$ & & $\times$ & & $\times \times \times$ & $\times$ & & & & \\
\hline $1_{4}$ & $\times$ & & $\times \times \times$ & & & & & & \\
\hline $2_{1}$ & $\times$ & & $\times$ & & $\times$ & $\begin{array}{c}\times \times \\
\times \times\end{array}$ & & & \\
\hline $2_{2}$ & & $\times$ & & & $\begin{array}{c}\times \times \\
\times \times\end{array}$ & $\times$ & & & \\
\hline 2 & & & & & & & & $\times \times \times$ & $\times$ \\
\hline 2 & & & & & & $\begin{array}{c}\times \times \times \\
\times \times\end{array}$ & & $\times$ \\
\hline 2 & & & & & & $\times$ & $\times$ & $\begin{array}{c}\times \\
\times\end{array}$ \\
\hline
\end{tabular}


The only $o(\Gamma)=27$ model to evaluate is $27 / 4$ with $\mathbf{4}=\left(\mathbf{1}_{2}, \mathbf{3}_{1}\right)$, where $\mathbf{6}=\mathbf{3}_{1}+\mathbf{3}_{2}$ is real, and where the scalar sector is:

\begin{tabular}{|c||c|c|c|c|c|c|c|c|c|c|c|}
\hline$\otimes$ & $1_{1}$ & $1_{2}$ & $1_{3}$ & $1_{4}$ & $1_{5}$ & $1_{6}$ & $1_{7}$ & $1_{8}$ & $1_{9}$ & $3_{1}$ & $3_{2}$ \\
\hline \hline $1_{1}$ & & & & & & & & & & $\times$ & $\times$ \\
\hline $1_{2}$ & & & & & & & & & & $\times$ & $\times$ \\
\hline $1_{3}$ & & & & & & & & & & $\times$ & $\times$ \\
\hline $1_{4}$ & & & & & & & & & & $\times$ & $\times$ \\
\hline $1_{5}$ & & & & & & & & & & $\times$ & $\times$ \\
\hline $1_{6}$ & & & & & & & & & & $\times$ & $\times$ \\
\hline $1_{7}$ & & & & & & & & & & $\times$ & $\times$ \\
\hline $1_{8}$ & & & & & & & & & & $\times$ & $\times$ \\
\hline $1_{9}$ & & & & & & & & & & $\times$ & $\times$ \\
\hline $3_{1}$ & $\times$ & $\times$ & $\times$ & $\times$ & $\times$ & $\times$ & $\times$ & $\times$ & $\times$ & & $\times \times \times$ \\
\hline $3_{2}$ & $\times$ & $\times$ & $\times$ & $\times$ & $\times$ & $\times$ & $\times$ & $\times$ & $\times$ & $\times \times \times$ & \\
\hline
\end{tabular}


And finally, at order 30 we have for $30 / 2$ with $\mathbf{4}=\left(\mathbf{1} \alpha, \mathbf{1}^{\prime}, \mathbf{2} \alpha\right)$ and $\mathbf{6}=\left(\mathbf{1}^{\prime} \alpha+\mathbf{2} \alpha+\mathbf{2} \alpha^{-1}+\mathbf{1}^{\prime} \alpha^{-1}\right)$ where $\alpha^{3}=1$, a model with scalar sector:

\begin{tabular}{|c||c|c|c|c|c|c|c|c|c|c|c|c|}
\hline$\otimes$ & 1 & $1^{\prime}$ & 2 & $2^{\prime}$ & $1 \alpha$ & $1^{\prime} \alpha$ & $2 \alpha$ & $2^{\prime} \alpha$ & $1 \alpha^{2}$ & $1^{\prime} \alpha^{2}$ & $2 \alpha^{2}$ & $2^{\prime} \alpha^{2}$ \\
\hline \hline 1 & & & & & & $\times$ & $\times$ & & & $\times$ & $\times$ & \\
\hline $1^{\prime}$ & & & & & $\times$ & & $\times$ & & $\times$ & & $\times$ & \\
\hline 2 & & & & & $\times$ & $\times$ & $\times$ & $\times$ & $\times$ & $\times$ & $\times$ & $\times$ \\
\hline $2^{\prime}$ & & & & & & & $\times$ & $\times \times$ & & & $\times$ & $\times \times$ \\
\hline $1 \alpha$ & & $\times$ & $\times$ & & & & & & & $\times$ & $\times$ & \\
\hline $1^{\prime} \alpha$ & $\times$ & & $\times$ & & & & & & $\times$ & & $\times$ & \\
\hline $2 \alpha$ & $\times$ & $\times$ & $\times$ & $\times$ & & & & & $\times$ & $\times$ & $\times$ & $\times$ \\
\hline $2^{\prime} \alpha$ & & & $\times$ & $\times \times$ & & & & & & & $\times$ & $\times \times$ \\
\hline $1 \alpha^{2}$ & & $\times$ & $\times$ & & & $\times$ & $\times$ & & & & & \\
\hline $1^{\prime} \alpha^{2}$ & $\times$ & & $\times$ & & $\times$ & & $\times$ & & & & & \\
\hline $2 \alpha^{2}$ & $\times$ & $\times$ & $\times$ & $\times$ & $\times$ & $\times$ & $\times$ & $\times$ & & & & \\
\hline $2^{\prime} \alpha^{2}$ & & & $\times$ & $\times \times$ & & & $\times$ & $\times \times$ & & & & \\
\hline
\end{tabular}


The other possibility at order 30 is $30 / 3$ with $\mathbf{4}=\left(\mathbf{1} \alpha, \mathbf{1}^{\prime}, \mathbf{2} \alpha^{2}\right)$ where $\mathbf{6}=\mathbf{1}^{\prime} \alpha+\mathbf{2} \alpha^{2}+\mathbf{2} \alpha^{3}+\mathbf{1}^{\prime} \alpha^{4}$ and $\alpha^{5}=1$, where the scalars are:

\begin{tabular}{|c||c|c|c|c|c|c|c|c|c|c|c|c|c|c|c|}
\hline$\otimes$ & 1 & $1^{\prime}$ & 2 & $1 \alpha$ & $1^{\prime} \alpha$ & $2 \alpha$ & $1 \alpha^{2}$ & $1^{\prime} \alpha^{2}$ & $2 \alpha^{2}$ & $1 \alpha^{3}$ & $1^{\prime} \alpha^{3}$ & $2 \alpha^{3}$ & $1 \alpha^{4}$ & $1^{\prime} \alpha^{4}$ & $2 \alpha^{4}$ \\
\hline \hline 1 & & & & & $\times$ & & & & $\times$ & & & $\times$ & & $\times$ & \\
\hline $1^{\prime}$ & & & & $\times$ & & & & & $\times$ & & & $\times$ & $\times$ & & \\
\hline 2 & & & & & & $\times$ & $\times$ & $\times$ & $\times$ & $\times$ & $\times$ & $\times$ & & & $\times$ \\
\hline $1 \alpha$ & & $\times$ & & & & & & $\times$ & & & & $\times$ & & & $\times$ \\
\hline $1^{\prime} \alpha$ & $\times$ & & & & & & $\times$ & & & & & $\times$ & & & $\times$ \\
\hline $2 \alpha$ & & & $\times$ & & & & & & $\times$ & $\times$ & $\times$ & $\times$ & $\times$ & $\times$ & $\times$ \\
\hline $1 \alpha^{2}$ & & & $\times$ & & $\times$ & & & & & & $\times$ & & & & $\times$ \\
\hline $1^{\prime} \alpha^{2}$ & & & $\times$ & $\times$ & & & & & & $\times$ & & & & & $\times$ \\
\hline $2 \alpha^{2}$ & $\times$ & $\times$ & $\times$ & & & $\times$ & & & & & & $\times$ & $\times$ & $\times$ & $\times$ \\
\hline $1 \alpha^{3}$ & & & $\times$ & & & $\times$ & & $\times$ & & & & & & $\times$ & \\
\hline $1^{\prime} \alpha^{3}$ & & & $\times$ & & & $\times$ & $\times$ & & & & & & $\times$ & & \\
\hline $2 \alpha^{3}$ & $\times$ & $\times$ & $\times$ & $\times$ & $\times$ & $\times$ & & & $\times$ & & & & & & $\times$ \\
\hline $1 \alpha^{4}$ & & $\times$ & & & & $\times$ & & & $\times$ & & $\times$ & & & & \\
\hline $1^{\prime} \alpha^{4}$ & $\times$ & & & & & $\times$ & & & $\times$ & $\times$ & & & & & \\
\hline $2 \alpha^{4}$ & & & $\times$ & $\times$ & $\times$ & $\times$ & $\times$ & $\times$ & $\times$ & & & $\times$ & & & \\
\hline
\end{tabular}

This concludes the enumeration of the scalar sectors of the properly embedded models. We will now investigate a number of symmetry breaking scenarios for these models. 


\subsection{Spontaneous Symmetry Breaking}

We are now in a position to carry out the spontaneous symmetry breaking for the models with fermions and scalars given in the previous sections. We restrict ourselves to chiral models with the potential of at least three families $(\chi \geq 45)$ and for the most part consider only models with $N=2$, although we have included two $N=3$ models. Again, we move progressively through the models of increasing order of $\Gamma$. The model is completely fixed by $\Gamma$, the embedding of 4 in $\Gamma$, and the choice of $N$.

It is worth mentioning explicitly that no attempt is made to stationarize the scalar potential in any of our models. We are tacitly assuming that there are sufficient parameters in the potential to arrange a minimum with the scalar VEVs displayed. It would be an interesting research project to study this in specific cases and will generally require a computer analysis.

The first relevant model is:

$$
\begin{aligned}
& \frac{16 / 8 \text { with } \mathbf{4}=\left(\mathbf{2}_{\mathbf{1}}, \mathbf{2}_{\mathbf{1}}\right) \text { and } N=2}{\text { The chiral fermions are }} \\
& 2[(2,1,1,1,1,1,1,1 ; 4,1)+(1,1,1,1,2,1,1,1 ; 1,4) \\
+ & (1,2,1,1,1,1,1,1 ; 4,1)+(1,1,1,1,1,2,1,1 ; 1,4) \\
+ & (1,1,2,1,1,1,1,1 ; 4,1)+(1,1,1,1,1,1,2,1 ; 1,4) \\
+ & (1,1,1,2,1,1,1,1 ; 4,1)+(1,1,1,1,1,1,1,2 ; 1,4) \\
+ & (2,1,1,1,1,1,1,1 ; 1, \overline{4})+(1,1,1,1,2,1,1,1 ; \overline{4}, 1) \\
+ & (1,2,1,1,1,1,1,1 ; 1, \overline{4})+(1,1,1,1,1,2,1,1 ; \overline{4}, 1) \\
+ & (1,1,2,1,1,1,1,1 ; 1, \overline{4})+(1,1,1,1,1,1,2,1 ; \overline{4}, 1) \\
+ & (1,1,1,2,1,1,1,1 ; 1, \overline{4})+(1,1,1,1,1,1,1,2 ; \overline{4}, 1)]
\end{aligned}
$$

and $\chi=2^{8}$. From the table of scalars for this model, we find that if we break $S U(4) \times S U(4)$ to the diagonal $S U_{D}(4)$, then the model becomes vectorlike.

All scalars that are nontrivial in the $S U(4)$ s are of the form $(1,1,1,1,1,1,1,1 ; 4, \overline{4})+$ h.c., and a VEV for any one can be 
rotated such that the unbroken symmetry is $S U_{D}(4)$. All other scalars are $S U_{i}(2) \times S U_{j}(2)$ bilinears, hence we cannot break to a Pati-Salam (PS) model or any standard type chiral model with this strategy.

As mentioned above, symmetry breaking via non-diagonal subgroups is another possibility, but we will not investigate such models here. They usually lead to somewhat more complicated patterns of spontaneous symmetry breaking (SSB) and while they are more cumbersome to analyze, they do offer further model building opportunities.

We continue with $16 / 8$ with $\mathbf{4}=\left(\mathbf{1}_{\mathbf{2}}, \mathbf{1}_{\mathbf{4}+\mathbf{i}}, \mathbf{2}_{\mathbf{1}}\right)$ and $N=2$, where

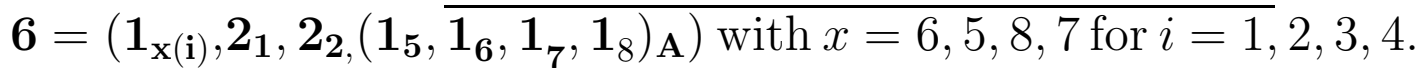
This model has only half the initial chirality of the previous model $\left(\chi=2^{7}\right)$, and the fermions are given above if the overall factor of 2 is removed. As above, we need to break one $S U(4)$, either will do. We choose $S U_{2}(4)$. For the scalars shown, we can do this with, say, $(1,1,1,2,1,1,1,1 ; 1, \overline{4})$ and $(1,1,1,1,1,1,1,2 ; 1,4)$ VEVs. The remaining chiral fermion sector is

$$
\begin{aligned}
& (2,1,1,1,1,1 ; 4)+(1,1,1,2,1,1 ; \overline{4})+(1,2,1,1,1,1 ; 4) \\
+ & (1,1,1,1,2,1 ; \overline{4})+(1,1,2,1,1,1 ; 4)+(1,1,1,1,1,2 ; \overline{4}) \\
& \text { for } G=\prod_{k} S U_{k}(2) \times S U(4), \text { with } \mathrm{k}=1,2,3,5,6,7 .
\end{aligned}
$$

There are only $S U_{i}(2) \times S U_{j}(2)$ bilinear scalars of the form $\left(2_{i}, 2_{j}\right)$ where $i=1,2$, or 3 and $j=4,5$,or 6 , whose VEVs reduce chirality further, so we cannot reach a three-family P-S model.

Note: what one would need is bilinears that allow us to break $S U_{1}(2) \times S U_{2}(2) \times S U_{3}(2)$ to a diagonal subgroup $S U_{L}(2)$, and similarly for $S U_{4}(2) \times S U_{5}(2) \times S U_{6}(2)$ to $S U_{R}(2)$. This would then have been a three-family P-S model, however, such scalars do not exist in the model.

Next consider $16 / 11$ with $\mathbf{4}=\left(\mathbf{1}_{\mathbf{2}}, \mathbf{1}_{\mathbf{2}}, \mathbf{1}_{\mathbf{2}}, \mathbf{1}_{\mathbf{2}}\right)$ and $N=3$. This model is highly chiral, with $\chi=432$, and the chiral fermions are 


$$
\begin{aligned}
& 6[(3, \overline{3}, 1,1,1,1,1,1 ; 1,1)+(1,1,1,1,3, \overline{3}, 1,1 ; 1,1) \\
+ & (1,3, \overline{3}, 1,1,1,1,1 ; 1,1)+(1,1,1,1,1,3, \overline{3}, 1 ; 1,1) \\
+ & (1,1,3, \overline{3}, 1,1,1,1 ; 1,1)+(1,1,1,1,1,1,3, \overline{3} ; 1,1) \\
+ & (\overline{3}, 1,1,3,1,1,1,1 ; 1,1)+(1,1,1,1, \overline{3}, 1,1,3 ; 1,1)] .
\end{aligned}
$$

We can ignore the $S U(6) \times S U(6)$ sector, since it can be broken completely without affecting the chirality. If we then give VEVs to $(1,1,1,8,1,1,1,1)$ and $(1,1,1,1,1,1,1,8)$ representations of $S U(3)^{8}$, we arrive at $6[(3, \overline{3}, 1)+(1,3, \overline{3})+(1,1,3)+$ $(\overline{3}, 1,1)]$ in the $S U_{i+1}(3) \times S U_{i+2}(3) \times S U_{i+3}(3)$ sector for both $i=0$ and $i=1$. The $i=0$ sector can be broken completely with $(1,1,1,1,8,1)$-type VEVs plus $(1,1,1,3,1, \overline{3})$-type VEVs. The remaining fermions falling nearly into six $E_{6} \longrightarrow$ $S U(3) \times S U(3) \times S U(3)$-type families. While close to containing the standard model, this model is still unsuccessful.

$16 / 11$ with $\mathbf{4}=\left(\mathbf{1}_{\mathbf{2}}, \mathbf{1}_{\mathbf{2}}, \mathbf{1}_{\mathbf{2}}, \mathbf{1}_{\mathbf{4}}\right)$ and $N=3$ The chiral fermion sector is exactly half the previous case. Again we break $S U(6) \times$ $S U$ (6) completely. Then breaking $\prod_{j=4}^{8} S U_{j}(3)$ completely with $S U_{j}(3)$ octet $\mathrm{VEV}$ gives us finally a chiral fermion sector $3[(3, \overline{3}, 1)+$ $(1,3, \overline{3})+(1,1,3)+(\overline{3}, 1,1)]$. This is tantalizingly close to a threefamily trinification model, but still lacks the requisite $(\overline{3}, 1,3)$ fermions.

16/13: There are three potential models for this group.

Consider first the case with

$\mathbf{4}=\left(2_{1}, 2_{1}\right)$ and $N=2$.

Here $\mathbf{6}=\left(1_{2}, 1_{2}, 1_{2}, 1_{3}, 2_{2}\right)$ and the chiral fermions are

$$
2[(2,1,1,1 ; 4,1,1)+(1,2,1,1 ; 1,1,4)+(1,1,2,1 ; 1,1,4)+
$$

$(1,1,1,2 ; 4,1,1)+(2,1,1,1 ; 1,1, \overline{4})+(1,2,1,1 ; \overline{4}, 1,1)$

$+(1,1,2,1 ; \overline{4}, 1,1)+(1,1,1,2 ; 1,1, \overline{4})]$ 
VEVs of the form $<4_{2}, \overline{4}_{2}>$ etc., can break $S U_{2}(4)$ completely (this group is irrelevant, since there are no chiral fermions with $S U_{2}(4)$ quantum numbers). VEVs for $\left(4_{1}, \overline{4}_{3}\right)$ scalars then breaks $S U_{1}(4) \times S U_{3}(4)$ to $S U_{D}(4)$, such that the fermions become vectorlike. On the other hand, VEVs for $\left(22_{4}, 4_{2}\right)+$ h.c. reduces the chiral sector to

$$
\begin{aligned}
& 2[(2,1,1 ; 1,4)+(1,2,1 ; 4,1)+(1,1,2 ; 4,1)+2(1,1,1 ; 1,4) \\
+ & (2,1,1 ; \overline{4}, 1)+2(1,1,1 ; \overline{4}, 1)+(1,2,1 ; 1, \overline{4})+(1,1,2 ; 1, \overline{4})]
\end{aligned}
$$

and then a VEV for $\left(22_{3}, 4_{2}\right)+$ h.c reduces this farther to $2[(2,1 ; 1,4)+(1,2 ; 4,1)+(1,2 ; 1, \overline{4})+(2,1 ; \overline{4}, 1)]$.

As above a VEV for $\left(4_{1}, \overline{4}_{3}\right)$ scalars would render the model vectorlike, while just breaking $S U_{3}(4)$ would give a one-family model. However, this needs VEVs for $\left(2_{1}, 2_{4}\right)$ and $\left(2_{2}, 2_{3}\right)$, but no scalars of this type exist in the model. We conclude the model has no Pati-Salam type phenomology.

\section{Consider next}

16/13 with $4=\left(\mathbf{2}_{1}, \mathbf{2}_{2}\right)$ and $N=2$.

This time $\mathbf{6}$ is as given in Section 6.5, but undetermined up to the identification of antisymmetric singlets in $\left(\mathbf{2}_{i} \times \mathbf{2}_{i}\right)_{A}$ with $i=$ 1,2 . The chiral fermions are as in the $\mathbf{4}=\left(\mathbf{2}_{\mathbf{1}}, \mathbf{2}_{\mathbf{1}}\right)$ case, but with the overall factor of 2 deleted. A useful strategy is to do a generic spontaneous symmetry breaking analysis to try to obtain a realistic Pati-Salam type phenomenology and then, if successful, one asks if the scalars to carry out the breaking are in the model. As above, $S U_{2}(4)$ is irrelevant and so can be ignored. If we identify $S U_{1}(2) \times S U_{4}(2)$ with $S U_{L}(2)$ and $S U_{2}(2) \times S U_{3}(2)$ with $S U_{R}(2)$, we find $2[(2,1 ; 1,4)+(1,2 ; 4,1)+(1,2 ; 1, \overline{4})+(2,1 ; \overline{4}, 1)]$. Now breaking one of the remaining $S U(4) s$ completely gives two families, and this is the best one can do. Hence independent of what scalars are available, there is no chance to get a model with three or more families.

The remaining 16/13 case is: 
$\mathbf{4}=\left(\mathbf{1}_{\mathbf{3}}, \mathbf{1}_{\mathbf{4}}, \mathbf{2}_{\mathbf{1}}\right)$ with $N=2$.

Now $\mathbf{6}=\left(\mathbf{1}_{\mathbf{2}}, \mathbf{2}_{\mathbf{1}}, \mathbf{2}_{\mathbf{3}} \mathbf{1}_{\mathbf{c}}\right)$. but the chiral fermions are in the same representations as the previous model, and so we can immediately conclude on general grounds that there is no viable phenomenology for this case.

\section{$18 / 3$}

Now consider

$18 / 3$ with $\mathbf{4}=\left(\mathbf{1} \alpha, \mathbf{1}^{\prime} ;, \mathbf{2} \alpha\right)$ and $N=3$. This model has $\chi=$ 192 and chiral fermions $(2,1,1,1,1,1 ; 1,4,1)+(1,2,1,1,1,1 ; 1,4,1)+$ $(1,1,2,1,1,1 ; \overline{4}, 1,1)$ $+(1,1,1,2,1,1 ; \overline{4}, 1,1)+(1,1,2,1,1,1 ; 1,1,4)+(1,1,1,2,1,1 ; 1,1,4)$ $+(1,1,1,1,2,1 ; 1, \overline{4}, 1)+(1,1,1,1,1,2 ; 1, \overline{4}, 1)+(1,1,1,1,2,1 ; 4,1,1)$ $+(1,1,1,1,1,2 ; 4,1,1)+(2,1,1,1,1,1 ; 1,1, \overline{4})+(1,2,1,1,1,1 ; 1,1, \overline{4})$ $+2[(1,1,1,1,1,1 ; \overline{4}, 4,1)+(1,1,1,1,1,1 ; 1, \overline{4}, 4)+(1,1,1,1,1,1 ; 4,1, \overline{4})]$. Breaking $S U^{6}(2)$ to a single diagonal $S U(2)$ with all six $\left(2_{i}, 2_{j}\right)$ type VEVs of $S U_{i}(2) \times S U_{j}(2)$, and then further VEVs of the type $(2 ; 4,1,1),(2 ; 1,4,1)$, and $(2 ; 1,1,4)$ to break the $S U(4)$ s to $S U(3)$ s leads to the set of remaining chiral fermions:

$2[(3, \overline{3}, 1)+(1,3, \overline{3})+(\overline{3}, 1,3)]$.

So this route leads to two families.

If instead we seek a Pati-Salam model, there are several spontaneous symmetry breaking routes we need to investigate. If we break with $(1,1,1,1,1,1 ; \overline{4}, 4,1)$ scalars to $S U^{6}(2) \times S U_{D}(4) \times$ $S U_{3}(4)$ we find the fermions remaining chiral are

$(2,1,1,1,1,1 ; 4,1)+(1,2,1,1,1,1 ; 4,1)+(1,1,2,1,1,1 ; 1,4)+$ $(1,1,1,2,1,1 ; 1,4)$

$+(1,1,2,1,1,1 ; \overline{4}, 1)+(1,1,1,2,1,1 ; \overline{4}, 1)+(2,1,1,1,1,1 ; 1, \overline{4})+$ $(1,2,1,1,1,1 ; 1, \overline{4})$.

Now breaking with a $\left(4_{1}, \overline{4}_{3}\right)$ or $\left(4_{2}, \overline{4}_{3}\right)$ VEV would render the model vectorlike, so we avoid this and insted give VEVs to 
$\left(2_{5}, 4_{1}\right)$ and $\left(22_{6}, 4_{1}\right)$ to break $S U_{D}(4)$ to $S U^{\prime}(2)$. However, this yields at most two families.

We must try another route. If we avoid $(\overline{4}, 4)$ type VEVs and give VEVs only to $(2,4)$ type scalars, we can proceed as follows: $<2_{1}, 4_{2}>,<2_{2}, 4_{2}>,<2_{3}, \overline{4}_{1}>$ and $<2_{4}, \overline{4}_{1}>\mathrm{VEVs}$ break $S U^{6}(2) \times S U^{3}(4)$ to $S U_{5}(2) \times S U_{6}(2) \times S U^{\prime}(2) \times S U^{\prime \prime}(2) \times$ $S U(4)$. Some fermions remain chiral but they are insufficient to construct families. We conclude that this model will not provide viable phenomenology.

$20 / 5$ with $\mathbf{4}=\left(\mathbf{1}_{\mathbf{2}}, \mathbf{1}_{\mathbf{2}}, \mathbf{1}_{\mathbf{2}}, \mathbf{1}_{\mathbf{2}}\right)$ and $N=3$

The chiral $S U^{4}(3)$ fermions are $4[(3, \overline{3}, 1,1)+(1,3, \overline{3}, 1)+$ $(1,1,3, \overline{3})+(\overline{3}, 1,1,3)]$. (The $S U(6)$ does not participate; it will be ignored.) The only scalars are in representations $(3,1, \overline{3}, 1)$ +h.c. and $(1,3,1, \overline{3})+$ h.c. A VEV to, say, the first of these, would break $S U_{1}(3) \times S U_{3}(3)$ to a diagonal $S U_{D}(3)$, and the fermions would become $4[(3, \overline{3}, 1)+(\overline{3}, 3,1)+(3,1, \overline{3})+(\overline{3}, 1,3)]$ under $S U_{D}(3) \times S U_{2}(3) \times S U_{4}(3)$, which is vectorlike. Hence any allowed VEVs immediately renders the model vectorlike.

We get no farther with $\mathbf{4}=\left(\mathbf{1}_{\mathbf{2}}, \mathbf{1}_{\mathbf{2}}, \mathbf{1}_{\mathbf{2}}, \mathbf{1}_{\mathbf{2}}\right)$ and $\mathrm{N}=3$, where $\mathbf{6}=\left(\mathbf{1}_{\mathbf{3}}, \mathbf{1}_{\mathbf{3}}, \mathbf{1}_{\mathbf{3}}, \mathbf{1}_{\mathbf{1}}, \mathbf{1}_{\mathbf{1}}, \mathbf{1}_{\mathbf{1}}\right)$, since this model has only half the chirality content of the previous case, and again VEVs will render it vectorlike.

$21 / 2$ with $4=\left(\mathbf{1}_{2}, \mathbf{3}_{1}\right)$ and $N=2$. Now $\mathbf{6}=\left(\mathbf{3}_{1}, \mathbf{3}_{2}\right)$. (Other embeddings of the 4 with $n=0$ SUSY are permutation of the representations of this model and are therefore all equivalent.) The fermions have $\chi=108$ and are $(2,1,1 ; 6,1)+(1,2,1 ; 6,1)+$ $(1,1,2 ; 6,1)+(2,1,1 ; 1, \overline{6})+(1,2,1 ; 1, \overline{6})+(1,1,2 ; 1, \overline{6})+(1,1,1 ; \overline{6}, 6)$. A VEV for a $(\overline{6}, 6)$ scalar renders the model vectorlike. Our only other option is to give $(2,6)$ type VEVs. A $<2,1,1 ; 6,1>$ breaks the gauge group to $S U_{2}(4) \times S U_{3}(2) \times S U(5) \times S U(6)$ with chiral fermions $2(1,1 ; 5,1)+(1,2 ; 5,1)+(2,1 ; 5,1)+(1,1 ; 1, \overline{6})+$ $(2,1 ; 1, \overline{6})+(1,2 ; 1, \overline{6})+(1,1,1 ; \overline{5}, 6)$. There is insufficient fermion content for a three family Pati-Salam model if we identify $S_{2}(4) \times$ 
$S U_{3}(2)$ with $S U_{L}(4) \times S U_{R}(2)$. Our only other choice is to get one of these $S U(2)$ s from $S U(5) \times S U(6)$. For instance a $<2_{2}, 5>\mathrm{VEV}$ breaks the gauge group to $S U_{3}(2) \times S U(4) \times S U(6)$ but the remaing chiral fermions are $4(1,4,1)+(2,4,1)+3(1,1, \overline{6})+(2,1, \overline{6})+$ $(1,2 ; 1, \overline{6})+(1,1,6)+(1, \overline{4}, 6)$. We can not identify $S U(4)$ with $S U_{P S}(4)$, so this group can only be in $S U(6)$. Breaking $S U(6)$ with an adjoint to $S U(2) \times S U(4)$ leaves us with $S U(2) \times S U(4) \times$ $S U(2) \times S U(4)$ fermions that are again insufficient for a three family Pati-Salam model.

$24 / 7$ with $\mathbf{4}=\left(\mathbf{1} \alpha, \mathbf{1}^{\prime}, \mathbf{2} \alpha\right)$ for $N=2$

This model, the only successful one in the present rather broad yet still not comprehensive search, has been discussed in detail in [178].

The original gauge group at the conformality scale is $S U(4)^{3} \times$ $S U(2)^{12}$ with chiral fermions as given in Section 6.4 and complex scalars as stated in Section 6.5 above.

If we break the three $S U(4)$ s to a single diagonal $S U(4)$ subgroup, chirality is lost. To avoid this we break $S U(4)_{1}$ completely and then break $S U(4)_{\alpha} \times S U(4)_{\alpha^{2}}$ to its diagonal subgroup $S U(4)_{D}$. The appropriate VEVs are available as $\left[\left(4_{1}, 2_{b} \alpha^{k}\right)+\right.$ h.c.] with $b$ arbitrary but $k=1$ or $k=2$. The second step requires an $S U(4)_{D}$ singlet VEV from $\left(\overline{4}_{\alpha}, 4_{\alpha^{2}}\right)$ and/or $\left(4_{\alpha}, \overline{4}_{\alpha^{2}}\right)$. Once a choice is made for $b$ (we take $b=4$ ), the remaining fermions are, in an intuitive notation,:

$$
\sum_{\alpha=1}^{\alpha=3}\left[\left(2_{\alpha} \alpha, 1,4_{D}\right)+\left(1,2_{\alpha} \alpha^{-1}, \overline{4}_{D}\right)\right]
$$

which has the same content as a three family Pati-Salam model, though with a separate $S U(2)_{L} \times S U(2)_{R}$ per family.

To further reduce the symmetry we must arrange to break to a single $S U(2)_{L}$ and a single $S U(2)_{R}$. This is achieved by modifying step one where $S U(4)_{1}$ was broken. Consider the block diagonal decomposition of $S U(4)_{1}$ into $S U(2)_{1 L} \times S U(2)_{1 R}$. The 
representations $\left(2_{\alpha} \alpha, 4_{1}\right)$ and $\left(2_{\alpha} \alpha^{-1}, 4_{1}\right)$ decompose as $\left(2_{\alpha} \alpha, 4_{1}\right) \rightarrow$ $\left(2_{\alpha} \alpha, 2,1\right)+\left(2_{\alpha} \alpha, 1,2\right)$ and $\left(2_{\alpha} \alpha^{-1}, 4_{1}\right) \rightarrow\left(2_{\alpha} \alpha^{-1}, 2,1\right)+\left(2_{\alpha}^{-1}, 1,2\right)$. Now if we give VEVs of equal magnitude to the $\left(2_{a} \alpha, 2,1\right), a=$ $1,2,3$ and equal magnitude VEVs to the $\left(2_{a} \alpha^{-1}, 1,2\right), a=1,2,3$, we break $S U(2)_{1 L} \times \prod_{a=1}^{a=3} S U\left(2_{a} \alpha\right)$ to a single $S U(2)_{L}$ and we break $S U(2)_{1 R} \times \prod_{a=1}^{a=3} S U\left(2_{a} \alpha^{-1}\right)$ to a single $S U(2)_{R}$. Finally, VEVs for $\left(22_{4} \alpha, 2,1\right)$ and $\left(2{ }_{4} \alpha, 1,2\right)$ as well as $\left(2{ }_{4} \alpha^{-1}, 2,1\right)$ and $\left(2_{4} \alpha^{-1}, 1,2\right)$ ensure that both $S U\left(2_{4} \alpha\right)$ and $S U\left(2_{4} \alpha^{-1}\right)$ are broken and that only three families remain chiral. The final set of chiral fermions is then $3[(2,1,4)+(1,2, \overline{4})]$ with gauge symmetry $S U(2)_{L} \times S U(2)_{R} \times S U(4)_{D}$.

To achieve the final reduction to the standard model, an adjoint VEV from $\left(\overline{4}_{\alpha}, 4_{\alpha^{2}}\right)$ and/or $\left(4_{\alpha}, \overline{4}_{\alpha^{2}}\right)$ is used to break $S U(4)_{D}$ to $S U(3) \times U(1)$, and a right-handed doublet is used to break $S U(2)_{R}$.

$24 / 9$ with $\mathbf{4}=\left(\mathbf{1}_{1} \alpha, \mathbf{1}_{2} \alpha^{3}, \mathbf{2} \alpha^{2}\right)$ for $N=2$

The original gauge group at the conformality scale is $S U(4)^{4} \times$ $S U(2)^{8}$ with chiral fermions as given in Section 6.4 and complex scalars as stated in Section 6.5 above.

Achievement of chiral families under the Pati-Salam subgroup $S U(4) \times S U(2)_{L} \times S U(2)_{R}$ requires the identifications $S U(2)_{1_{1}}=$ $S U(2)_{1_{2}}=S U(2)_{1_{1} \alpha}=S U(2)_{1_{2} \alpha}=S U(2)_{L} ; S U(2)_{1_{1} \alpha^{3}}=S U(2)_{1_{2} \alpha^{2}}=$ $S U(2)_{1_{1} \alpha^{3}}=S U(2)_{1_{2} \alpha^{3}}=S U(2)_{R}$; while, for example, $S U(4)_{2}=$ $S U(4)_{2 \alpha}=\overline{4}$ of $S U(4) ; S U(4)_{2 \alpha^{2}}=S U(4)_{2 \alpha^{3}}=4$ of $S U(4)$ where by this simplified notation we imply diagonal subgroups.

But the scalars tabulated for this case in Section 6.5 are insufficient to allow this pattern of spontaneous symmetry breaking, and hence no interesting model emerges.

$24 / 9$ with $\mathbf{4}=\left(\mathbf{1}_{1} \alpha, \mathbf{1}_{2} \alpha, \mathbf{2} \alpha\right)$ for $N=2$

The original gauge group at the conformality scale is $S U(4)^{4} \times$ $S U(2)^{8}$ with chiral fermions as given in Section 6.4 and complex scalars as stated in Section 6.5 above. 
Achievement of chiral families under the Pati-Salam subgroup $S U(4) \times S U(2)_{L} \times S U(2)_{R}$ requires the identifications $S U(2)_{1_{1}}=$ $S U(2)_{1_{2}}=S U(2)_{1_{1} \alpha}=S U(2)_{1_{2} \alpha}=S U(2)_{L} ; S U(2)_{1_{1} \alpha^{3}}=S U(2)_{1_{2} \alpha^{2}}=$ $S U(2)_{1} \alpha^{3}=S U(2)_{1_{2} \alpha^{3}}=S U(2)_{R}$; while, for example, $S U(4)_{2}=$ $S U(4)_{2 \alpha^{3}}=\overline{4}$ of $S U(4) ; S U(4)_{2 \alpha}=S U(4)_{2 \alpha^{2}}=4$ of $S U(4)$ where again by this simplified notation we imply diagonal subgroups.

But the scalars tabulated for this case in Section 6.5 are insufficient to allow this pattern of spontaneous symmetry breaking, and hence no interesting model emerges.

$24 / 9$ with $\mathbf{4}=\left(\mathbf{1}_{1} \alpha^{2}, \mathbf{1}_{2}, \mathbf{2} \alpha\right)$ for $N=2$

The original gauge group at the conformality scale is $S U(4)^{4} \times$ $S U(2)^{8}$ with chiral fermions as given in Section 6.4 and complex scalars as stated in Section 6.5 above.

Achievement of chiral families under the Pati-Salam subgroup $S U(4) \times S U(2)_{L} \times S U(2)_{R}$ requires the identifications $S U(2)_{1_{1}}=$ $S U(2)_{1_{2}}=S U(2)_{1_{1} \alpha}=S U(2)_{1_{2} \alpha}=S U(2)_{L} ; S U(2)_{1_{1} \alpha^{3}}=S U(2)_{1_{2} \alpha^{2}}=$ $S U(2)_{1_{1} \alpha^{3}}=S U(2)_{1_{2} \alpha^{3}}=S U(2)_{R}$; while, for example, $S U(4)_{2}=$ $S U(4)_{2 \alpha^{3}}=\overline{4}$ of $S U(4) ; S U(4)_{2 \alpha}=S U(4)_{2 \alpha^{2}}=4$ of $S U(4)$ where by this simplified notation we imply diagonal subgroups.

But again, as in the previous two embeddings, the scalars tabulated for this case in Section 6.5 are insufficient to allow this pattern of spontaneous symmetry breaking, and hence no interesting model emerges. 
The final model to consider for this group is $24 / 9$ with $\mathbf{4}=(\mathbf{2} \alpha, \mathbf{2} \alpha)$ for $N=2$

The original gauge group at the conformality scale is $S U(4)^{4} \times$ $S U(2)^{8}$ with chiral fermions as given in Section 6.4 and complex scalars as stated in Section 6.5 above.

Achievement of chiral families under the Pati-Salam subgroup $S U(4) \times S U(2)_{L} \times S U(2)_{R}$ requires the identifications $S U(2)_{1_{1}}=$ $S U(2)_{1_{1} \alpha}=S U(2)_{1_{1} \alpha^{2}}=S U(2)_{1_{1} \alpha^{3}}=S U(2)_{L} ; S U(2)_{1_{2} \alpha}=$ $S U(2)_{1_{2} \alpha}=S U(2)_{1_{2} \alpha^{2}}=S U(2)_{1_{2} \alpha^{3}}=S U(2)_{R}$; while, for example, $S U(4)_{2 \alpha}=S U(4)_{2 \alpha^{3}}=4$ of $S U(4)$ where by this simplified notation we imply diagonal subgroups, and $S U(4)_{2}$ and $S U(4)_{2 \alpha^{2}}$ are broken.

But the scalars tabulated for this case in Section 6.5 are insufficient to allow this pattern of spontaneous symmetry breaking, and hence no interesting model emerges.

Moving on we next consider $24 / 13$ with $\mathbf{4}=\left(\mathbf{2}_{1}, \mathbf{2}_{2}\right)$ for $N=2$

The original gauge group at the conformality scale is $S U(6) \times$ $S U(4)^{3} \times S U(2)^{3}$ with chiral fermions as given in Section 6.4 and complex scalars as stated in Section 6.5 above.

According to the analysis in Section 6.4 this orbifold permits only two chiral families and is therefore not of phenomenological interest.

$24 / 14$ with $\mathbf{4}=\left(\mathbf{2}_{1}, \mathbf{2}_{2}\right)$ for $N=2$

The original gauge group at the conformality scale is $S U(4)^{4} \times$ $S U(2)^{8}$ with chiral fermions as given in Section 6.4 and complex scalars as stated in Section 6.5 above.

Achievement of chiral families under the Pati-Salam subgroup $S U(4) \times S U(2)_{L} \times S U(2)_{R}$ requires the identifications $S U(2)_{1_{1}}=$ $S U(2)_{1_{2}}=S U(2)_{1_{5}}=S U(2)_{1_{6}}=S U(2)_{L} ; S U(2)_{1_{3}}=S U(2)_{1_{4}}=$ $S U(2)_{1_{5}}=S U(2)_{1_{6}}=S U(2)_{R}$; while, for example, $S U(4)_{2_{2}}=$ 
$S U(4)_{2_{3}}=4$ of $S U(4) ; S U(4)_{2_{1}}=S U(4)_{2_{4}}=\overline{4}$ of $S U(4)$ where by this simplified notation we imply diagonal subgroups.

But the scalars tabulated for this case in Section 6.5 are insufficient to allow this pattern of spontaneous symmetry breaking, and hence no interesting model emerges.

\section{$24 / 15$ with $\mathbf{4}=\left(\mathbf{1}_{2}, \mathbf{1}_{3}, \mathbf{2}_{3}\right)$ for $N=2$}

The original gauge group at the conformality scale is $S U(4)^{5} \times$ $S U(2)^{4}$ with chiral fermions as given in Section 6.4 and complex scalars as stated in Section 6.5 above.

Achievement of chiral families under the Pati-Salam subgroup $S U(4) \times S U(2)_{L} \times S U(2)_{R}$ requires the identifications $S U(2)_{1_{1}}=S U(2)_{1_{3}}=S U(2)_{L} ; S U(2)_{1_{2}}=S U(2)_{1_{4}}=S U(2)_{R}$; while, for example, $S U(4)_{2_{3}}=S U(4)_{2_{4}}=4$ of $S U(4)$, where by this simplified notation we imply diagonal subgroups.

But again the scalars tabulated for this case in Section 6.5 are insufficient to allow this pattern of spontaneous symmetry breaking, and hence no interesting model emerges.

$24 / 15$ with $\mathbf{4}=\left(\mathbf{2}_{3}, \mathbf{2}_{5}\right)$ for $N=2$

The original gauge group at the conformality scale is $S U(4)^{5} \times$ $S U(2)^{4}$ with chiral fermions as given in Section 6.4 and complex scalars as stated in Section 6.5 above.

According to the analysis in Section 6.4 this orbifold permits only two chiral families and is hence not phenomenologically interesting.

$24 / 15$ with $4=\left(\mathbf{2}_{3}, \mathbf{2}_{3}\right)$ for $N=2$

The original gauge group at the conformality scale is $S U(4)^{5} \times$ $S U(2)^{4}$ with chiral fermions as given in Section 6.4 and complex scalars as stated in Section 6.5 above.

Achievement of chiral families under the Pati-Salam subgroup $S U(4) \times S U(2)_{L} \times S U(2)_{R}$ requires the identifications 
$S U(2)_{1_{1}}=S U(2)_{1_{3}}=S U(2)_{L} ; S U(2)_{1_{2}}=S U(2)_{1_{4}}=S U(2)_{R}$; while, for example, $S U(4)_{2_{3}}=S U(4)_{2_{4}}=4$ of $S U(4)$ where by this simplified notation we imply diagonal subgroups.

But the scalars tabulated for this case in Section 6.5 are insufficient to allow this pattern of spontaneous symmetry breaking, and hence no interesting model emerges.

Continuing on to $o(\Gamma)=27$ we have

$27 / 4$ with $\mathbf{4}=\left(\mathbf{1}_{2}, \mathbf{3}_{1}\right)$ with $N=2$.

Here $\mathbf{6}=\left(\mathbf{3}_{1}, \mathbf{3}_{2}\right)$ and the chiral fermions are given by Equation 49 and all scalars are of type $\left(2_{i}, \overline{6}_{1}\right),\left(2_{i}, 6_{2}\right)$ or $\left(6_{1}, \overline{6}_{2}\right)$ for $i=1,2, \ldots, 9$.. A VEV for $\left(6_{1}, \overline{6}_{2}\right)+$ h.c. scalar breaks $S U_{1}(6) \times$ $S U_{2}(6)$ to $S U_{D}(6)$, and the model becomes vectorlike. Hence we must break only with $(2,6)$ type scalars if there is any hope of a viable model. We give VEVs to $\left(2_{i}, 6_{1}\right)$ scalars for $i=$ $1,2, \ldots, 5$ to break $S U_{1}(6)$ completely, and VEVs to $\left(2_{j}, 6_{2}\right)$ for $j=6,7$ to break $S U_{2}(6)$ to $S U(4)$. Then the remaining unbroken gauge group is $S U_{8}(2) \times S U_{9}(2) \times S U(4)$ with fermions $(2,1,4)+(1,2,4)+4(1,1, \overline{4})$, which are chiral but not of the correct form.

A more successful variation is obtained with $\left(2_{i}, 6_{1}\right)$ scalars VEVs for $i=1,2,3$ and 4 to break the gauge group to $S U_{5}(2) \times$ $S U_{6}(2) \times S U_{7}(2) \times S U_{8}(2) \times S U_{9}(2) \times S U^{\prime}(2) \times S U(6)$ and thenVEVs for $\left(2_{5}, 6_{2}\right)$ and $\left(2_{6}, 6_{2}\right)$ to break to $S U_{7}(2) \times S U_{8}(2) \times$ $S U_{9}(2) \times S U^{\prime}(2) \times S U(4)$ which has chiral fermions $(2,1,1,1,4)+(1,2,1,1,4)+(1,1,2,1,4)+3(1,1,1,2,4)$. If we could break $S U_{7}(2) \times S U_{8}(2) \times S U_{9}(2)$ to a diagonal $S U(2)$ subgroup, we would have a three-family Pati-Salam model. However, the scalars to accomplish this are not in the spectrum. If we could give VEVs to $\left(2_{i}, 6_{1}\right)$ scalars for $i=7,8,9$ to break $S U_{7}(2) \times S U_{8}(2) \times S U_{9}(2)$ to a $U_{Y}(1)$ without disturbing the $S U^{\prime}(2)$ subgroup of $S U_{1}(6)$, and a further $\left(2_{j}, 6_{2}\right) \mathrm{VEV}$, say $\left(2_{1}, 6_{2}\right)$, to break $S U(4)$ to $S U_{C}(3)$, then we would have a true three family standard (i.e., $U_{Y}(1) \times S U_{E W}(2) \times S U_{C}(3)$ ) model 
upon identifying $S U^{\prime}(2)$ with $S U_{E W}(2)$.

Finally at $o(\Gamma)=30$ we have $30 / 2$ with $\mathbf{4}=\left(\mathbf{1}_{\mathbf{1}} \alpha, \mathbf{1}_{\mathbf{2}}, \mathbf{2} \alpha\right)$ and $N=2$.

Here $\mathbf{6}=\left(\mathbf{1}_{2} \alpha, \mathbf{1}_{2} \alpha^{\mathbf{2}}, \mathbf{2} \alpha, \mathbf{2} \alpha^{\mathbf{2}}\right)$, and the gauge group is $S U^{6}(2) \times$ $S U^{6}(4)$. This group has chiral fermions:

$(2,1,1,1,1,1 ; 1,1,4,1,1,1)+(1,2,1,1,1,1 ; 1,1,4,1,1,1)$

$+(1,1,2,1,1,1 ; \overline{4}, 1,1,1,1,1)+(1,1,1,2,1,1 ; \overline{4}, 1,1,1,1,1)$

$+(1,1,1,1,1,1 ; 1, \overline{4}, 4,1,1,1)+(1,1,1,1,1,1 ; \overline{4}, 1,4,1,1,1)$

$+2(1,1,1,1,1,1 ; 1, \overline{4}, 1,4,1,1)+(1,1,1,1,1,1 ; \overline{4}, 1,1,4,1,1)$

$+(1,1,2,1,1,1 ; 1,1,1,1,4,1)+(1,1,1,2,1,1 ; 1,1,1,1,4,1)$

$+(1,1,1,1,2,1 ; 1,1, \overline{4}, 1,1,1)+(1,1,1,1,1,2 ; 1,1, \overline{4}, 1,1,1)$

$+(1,1,1,1,1,1 ; 1,1,1, \overline{4}, 4,1)+(1,1,1,1,1,1 ; 1,1, \overline{4}, 1,4,1)$

$+2(1,1,1,1,1,1 ; 1,1,1, \overline{4}, 1,4)+(1,1,1,1,1,1 ; 1,1, \overline{4}, 1,1,4)$

$+(1,1,1,1,2,1 ; 4,1,1,1,1,1)+(1,1,1,1,1,2 ; 4,1,1,1,1,1)$

$+(2,1,1,1,1,1 ; 1,1,1,1, \overline{4}, 1)+(1,2,1,1,1,1 ; 1,1,1,1, \overline{4}, 1)$

$+(1,1,1,1,1,1 ; 4,1,1,1, \overline{4}, 1)+(1,1,1,1,1,1 ; 4,1,1,1,1, \overline{4})$

$+2(1,1,1,1,1,1 ; 1,4,1,1,1, \overline{4})+(1,1,1,1,1,1 ; 1,4,1,1, \overline{4}, 1)$

The spontaneous symmetry breaking analysis for this model is quite unwieldy, but for the most part can be carried out systematically. For example, breaking with $(1,1,1,1,1,1 ; 1, \overline{4}, 1,4,1,1),(1,1,1,1,1,1 ; \overline{4}, 1,1,4,1,1)$,

$(1,1,1,1,1,1 ; 4,1,1,1, \overline{4}, 1)$ and $(1,1,1,1,1,1 ; 1,1, \overline{4}, 1,1,4)$ VEVs reduces $S U^{6}(4)$ to $S U_{1}(4) \times S U_{D}(4)$, with fermions remaining chiral in representations:

$(2,1,1,1,1,1 ; 1,4)+(1,2,1,1,1,1 ; 1,4)+(1,1,2,1,1,1 ; \overline{4}, 1)+$ $(1,1,1,2,1,1 ; \overline{4}, 1)$

$+(1,1,2,1,1,1 ; 1,4)+(1,1,1,2,1,1 ; 1,4)+(1,1,1,1,2,1 ; 1, \overline{4})+$ $(1,1,1,1,1,2 ; 1, \overline{4})$ 
$+(1,1,1,1,2,1 ; 4,1)+(1,1,1,1,1,2 ; 4,1)+(2,1,1,1,1,1 ; 1, \overline{4})+$ $(1,2,1,1,1,1 ; 1, \overline{4})$.

Now $(1,1,1,1,2,1 ; 4,1)$ and $(1,1,1,1,1,2 ; 4,1)$ VEVs break $S U_{5}(2) \times$ $S U_{6}(2) \times S U_{1}(4)$ to $S U^{\prime}(2)$ with fermions remaining chiral in the representations:

$(2,1,1,1 ; 4)+(1,2,1,1 ; 4)+(1,1,2,1 ; 4)+(1,1,1,2 ; 4)$

$+2(1,1,1,1 ; \overline{4})+2(1,1,1,1 ; \overline{4})+(2,1,1,1 ; \overline{4})+(1,2,1,1 ; \overline{4})$

which is already insufficient to provide three normal families. Other analyses of spontaneous symmetry breaking toward constructing a Pati-Salam model starting with this 30/2 model are similarly unsucessful.

An alternative is to seek a trinification model. To this end, consider only the $S U^{6}(4)$ fermion sector

$$
\begin{aligned}
& +(1, \overline{4}, 4,1,1,1)+(\overline{4}, 1,4,1,1,1)+2(1, \overline{4}, 1,4,1,1) \\
+ & (\overline{4}, 1,1,4,1,1)+(1,1,1, \overline{4}, 4,1)+(1,1, \overline{4}, 1,4,1) \\
+ & +2(1,1,1, \overline{4}, 1,4)+(1,1, \overline{4}, 1,1,4)+(4,1,1,1, \overline{4}, 1) \\
+ & 2(4,1,1,1,1, \overline{4})+2(1,4,1,1,1, \overline{4})+(1,4,1,1, \overline{4}, 1)
\end{aligned}
$$

Identifying $S U_{1}(4)$ with $S U_{2}(4), S U_{3}(4)$ with $S U_{4}(4)$ and $S U_{5}(4)$ with $S U_{6}(4)$ would lead to five families of the form $5[(\overline{4}, 4,1)+$ $(1, \overline{4}, 4)+(4,1, \overline{4})]$, however there are no scalars of the type needed to carry this out.

This analysis is not exhaustive and there may be models where $S U_{L}(2), S U_{R}(2)$ or both are contained in $S U^{6}(4)$. Since we are starting with a group of rank 24, and seek the standard model of rank 4 or a unified model thereof of rank 5 or 6, and since there are 66 Higgs representations in the theory, the spontaneous symmetry breaking possibilities are rather complex. The $N=3$ case is obviously even more complicated, with initial rank 42, and one could try to automate the search for phenomenological models, although we have not attempted to do so.

$$
\begin{aligned}
& 30 / 3 \text { with } \mathbf{4}=\left(\mathbf{1}_{\mathbf{1}} \alpha, \mathbf{1}_{\mathbf{2}}, \mathbf{2} \alpha^{\mathbf{2}}\right) \text { and } N=2 \text {. We now have } \mathbf{6}= \\
& \left(\mathbf{1}_{2} \alpha, \mathbf{1}_{2} \alpha^{4}, \mathbf{2} \alpha^{3}, \mathbf{2} \alpha^{2}\right) \text { where } \alpha^{5}=\mathbf{1} \text {. }
\end{aligned}
$$


The chiral $S U^{10}(2) \times S U^{5}(4)$ fermions are

$$
\left(1^{10} ; \overline{4}, 4,1,1,1\right)+\left(1^{10} ; \overline{4}, 1,4,1,1\right)+\left(1^{4}, 2,1^{5} ; \overline{4}, 1,1,1,1\right)
$$

$+\left(1^{5}, 2,1^{4} ; \overline{4}, 1,1,1,1\right)+\left(1^{10} ; 1, \overline{4}, 4,1,1\right)+\left(1^{10} ; 1, \overline{4}, 1,4,1\right)$

$+\left(1^{6}, 2,1^{3} ; 1, \overline{4}, 1,1,1\right)+\left(1^{7}, 2,1^{2} ; 1, \overline{4}, 1,1,1\right)+\left(1^{10} ; 1,1, \overline{4}, 4,1\right)$

$+\left(1^{10} ; 1,1, \overline{4}, 1,4\right)+\left(1^{8}, 2,1^{1} ; 1,1, \overline{4}, 1,1\right)+\left(1^{9}, 2 ; 1,1, \overline{4}, 1,1\right)$

$+\left(1^{10} ; 1,1,1, \overline{4}, 4\right)+\left(1^{10} ; 4,1,1, \overline{4}, 1\right)+\left(2,1^{9} ; 1,1,1, \overline{4}, 1\right)$

$+\left(1^{1}, 2,1^{8} ; 1,1,1, \overline{4}, 1\right)+\left(1^{10} ; 4,1,1,1, \overline{4}\right)+\left(1^{10} ; 1,4,1,1, \overline{4}\right)+\left(1^{2}, 2,1^{7}\right.$;

$1,1,1,1, \overline{4})$

$+\left(1^{3}, 2,1^{6} ; 1,1,1,1, \overline{4}\right)$

Consider the bifundamentals only. VEVs for $(1,1,1, \overline{4}, 4)$ and $(1, \overline{4}, 4,1,1)$ scalars reduce the chiral fermion sector to $2[(\overline{4}, 4,1)+$ $(1, \overline{4}, 4)+(4,1, \overline{4})]$ which provides at most a two family model.

If instead we try to construct a Pati-Salam model, and note that there are $20(2 ; 4)$ type fermions, and that we need six appropriate ones of these for three families, we must take care in the spontaneous symmetry breaking to preserve this much chirality. If we (i), break $S U_{2}(4) \times S U_{4}(4) \times S U_{5}(4)$ completely and (ii) $S U_{1}(4) \times S U_{3}(4)$ to $S U_{P S}(4)$ while (iii) equating $S U_{5}(2)$, $S U_{6}(2), S U_{9}(2)$ and (iv) equating $S U_{10}(2)$ with $S U_{L}(2)$, and $S U_{1}(2), S U_{2}(2), S U_{7}(2)$ and $S U_{8}(2)$ with $S U_{R}(2)$, and (v) breaking $S U_{3}(2) \times S U_{4}(2)$ completely, we would be left with a 4 family Pati-Salam Fmodel. Can we do this? (ii) is accomplished with (a) $\left(1^{10} ; \overline{4}, 1,4,1,1\right)$, then (i) requires (b) $\left(1^{10} ; 1, \overline{4}, 1,4,1\right)$ and (c) $\left(1^{10} ; 1, \overline{4}, 1,1,4\right)$ to get a $S U_{D}(4)$. Breaking this to nothing, assuming VEVs (a) and (b) allow no freedom to rotate the (c) VEV to diagonal form. Now, at this point, we are stymied, as there are insufficient $\left(2_{i}, 2_{j}\right)$ representations of $S U_{i}(2) \times S U_{j}(2)$ to accomplish (v).

Finally, one can imagine that there exist models with either $S U_{L}(2)$ or $S U_{R}(2)$ or both coming from $S U^{5}(4)$, but we see not obvious way to cary this out, while on the other hand since there are 60 Higgs representations we are unable to categorically eliminate this possibility. 


\section{Summary}

We have shown how $A d S / C F T$ duality leads to a large class of models which can provide interesting extensions of the standard model of particle phenomenology. The naturally occurring $\mathcal{N}=4$ extended supersymmetry was completely broken to $\mathcal{N}=0$ by choice of orbifolds $S^{5} / \Gamma$ such that $\Gamma \not \subset S U(3)$.

In the present work, we studied systematically all such nonabelian $\Gamma$ with order $g \leq 31$. We have seen how chiral fermions require that the embedding of $\Gamma$ be neither real nor pseudoreal. This reduces dramatically the number of possibilities to obtain chiral fermions. Nevertheless, many candidates for models which contain the chiral fermions of the three-family standard model were found.

However, the requirement that the spontaneous symmetry breaking down to the correct gauge symmetry of the standard model be permitted by the prescribed scalar representations eliminates most of the surviving models. We found only one allowed model based on the $\Gamma=24 / 7$ orbifold. We had initially expected to find more examples in our search. The moral for model-building is interesting. Without the rigid framework of string duality the scalar sector would be arbitrarily chosen to permit the required spontaneous symmetry breaking. This is the normal practice in the standard model, in grand unification, in supersymmetry and so on. With string duality, the scalar sector is prescribed by the construction and only in one very special case does it permit the required symmetry breaking.

This leads us to give more credence to the $\Gamma=24 / 7$ example that does work and to encourage its further study to check whether it can have any connection to the real world.

Note that we have usually taken the minimal choice for $N$, either 2 or 3, and have only considered SSB through diagonal subgroup chains. Both these constraints can obviously be relaxed 
to generate more model building opportunities, but clearly this is an open ended process as is choosing an orbifolding group. If properly motivated, then one of these directions may be productive. We stress that we are providing a guide to the process of model building, and not a compendium of carefully selected models. 


\section{Unification}

Going through the abelian quiver gauge theories, we may search for a model that contains as a sub theory the states of the standard model.

The scalar sector is dictated by the construction so that one does not have the usual model building luxury of choosing those scalar represntations which will most conveniently break the symmetry spontaneously in the manner desired. The constraint that the scalars present can break the gauge symmetry to the standard model is an exceptionally strong constraint which is, in general, not satisfied.

For $p \leq 6$ there is no solution. Only when we arrive at $p=7$ there are viable models $[176,177]$. Actually three different $p=7$ quiver diagrams can give: 3 chiral families, adequate scalars to spontaneously break $U(3)^{7} \rightarrow S U(3) \times S U(2) \times U(1)$ and $\sin ^{2} \theta_{W}=3 / 13=0.231$

The possible embeddings of $\Gamma=Z_{7}$ in $\mathrm{SU}(4)$ are:

7A. $\left(\alpha, \alpha, \alpha, \alpha^{4}\right)$

7B. $\left(\alpha, \alpha, \alpha^{2}, \alpha^{3}\right)^{*} \quad$ C-H-H-H-W-H-W

7C. $\left(\alpha, \alpha^{2}, \alpha^{2}, \alpha^{2}\right)$

7D. $\left(\alpha, \alpha^{3}, \alpha^{5}, \alpha^{5}\right)^{*} \quad$ C-H-W-H-H-H-W

7E. $\quad\left(\alpha, \alpha^{4}, \alpha^{4}, \alpha^{5}\right)^{*} \quad$ C-H-W-W-H-H-H

7F. $\quad\left(\alpha^{2}, \alpha^{4}, \alpha^{4}, \alpha^{4}\right)$

The three marked ${ }^{*}$ are those which possess the desired properties, The corresponding scalar quivers are indicated in the following figures. 
$7 \mathrm{~B}$

$4=(1,1,2,3) \quad 6=(2,3,3,-3,-3,-2)$

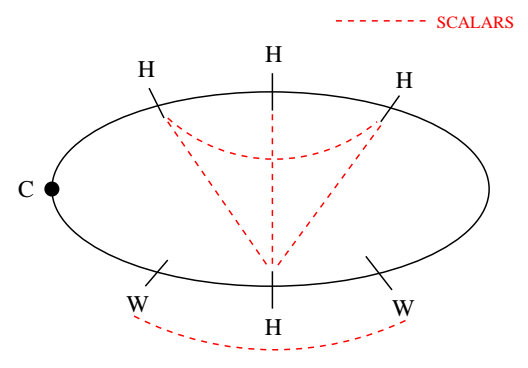

7D

$$
4=(1,3,5,5) \quad 6=(1,1,3,-3,-1,-1)
$$

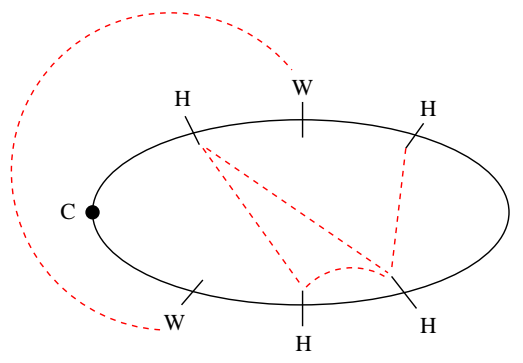

So the simplest abelian orbifold conformal extension of the standard model has $U(3)^{7} \rightarrow S U(3)^{3}$ trinification $\rightarrow(321)_{S M}$. In these cases we have $\alpha_{2}$ and $\alpha_{1}$ related correctly for low energy. But $\alpha_{3}(M) \simeq 0.07$ suggesting a conformal scale $M \geq 10 \mathrm{TeV}$ too high for detection at the L.H.C. The simple cases are not, in any cases, grand unified and far more interesting example uses $p=12$ and accommodates a TeV scale grand unification of a new type. The above merely illustrates how all the standard model states can be accommodated in abelian quiver theories.

$7 \mathrm{E} \quad 4=(1,4,4,5) \quad 6=(1,2,2,-2,-2,-1)$

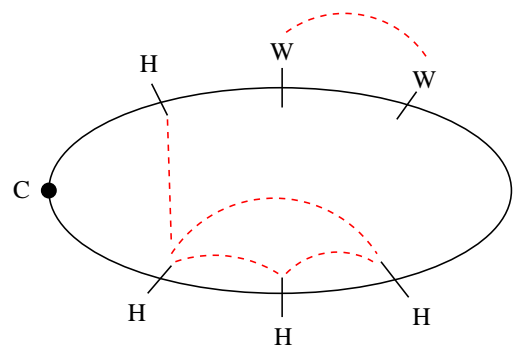




\subsection{Grand unification at $4 \mathrm{TeV}$}

A grand unified model [184] which introduces the $4 \mathrm{TeV}$ scale is: obtained by taking as orbifold $S^{5} / Z_{12}$ with embedding of $Z_{12}$ in the $\mathrm{SU}(4)$ R-parity specified by $\left.\mathbf{4} \equiv \alpha^{A_{1}}, \alpha^{A_{2}}, \alpha^{A_{3}}, \alpha^{A_{4}}\right)$ and $A_{\mu}=(1,2,3,6)$.

Firstly, this accommodates the scalars necessary to spontaneously break to the SM.

As a bonus, the dodecagonal quiver predicts three chiral families (see next page).

In this teravolt unification of $S U(3), S U(2)$ and $U(1)$ at $4 \mathrm{TeV}$, we shall assume that all non standard model states have masses satisfying $M \leq 4 \mathrm{TeV}$ and that the couplings of $S U(3)^{12}$ are scale invariant at higher energies. As will be discussed in Section 8 , cancellation of quadratic divergences requires that the $U(1)$ charged states also be at teravolt scales. A mechanism by which $U(1)$ couplings can (surprisingly) become scale invariant will be discussed in Section 9. 


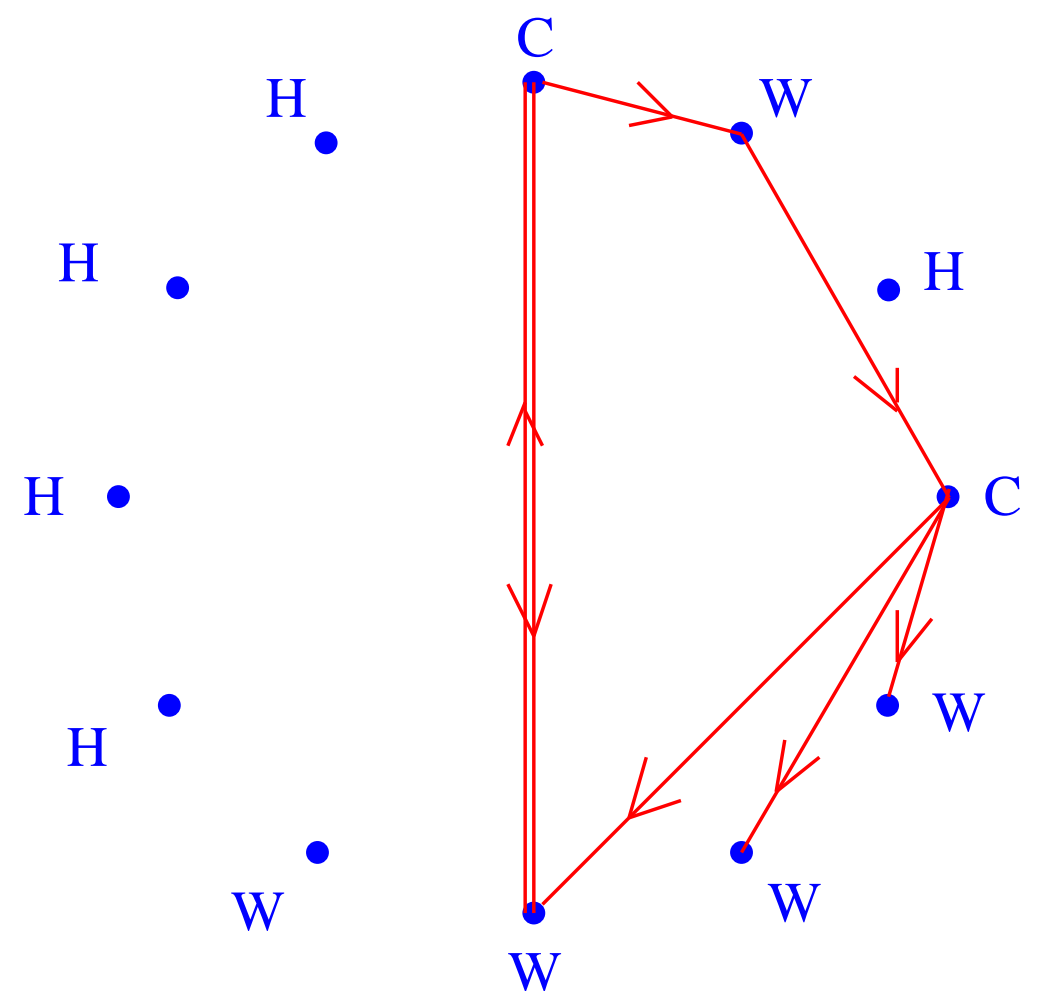

$$
\begin{aligned}
& A_{\mu}=(1,2,3,6) \\
& S U(3)_{C} \times S U(3)_{H} \times S U(3)_{H} \\
& 5(3, \overline{3}, 1)+2(\overline{3}, 3,1)
\end{aligned}
$$




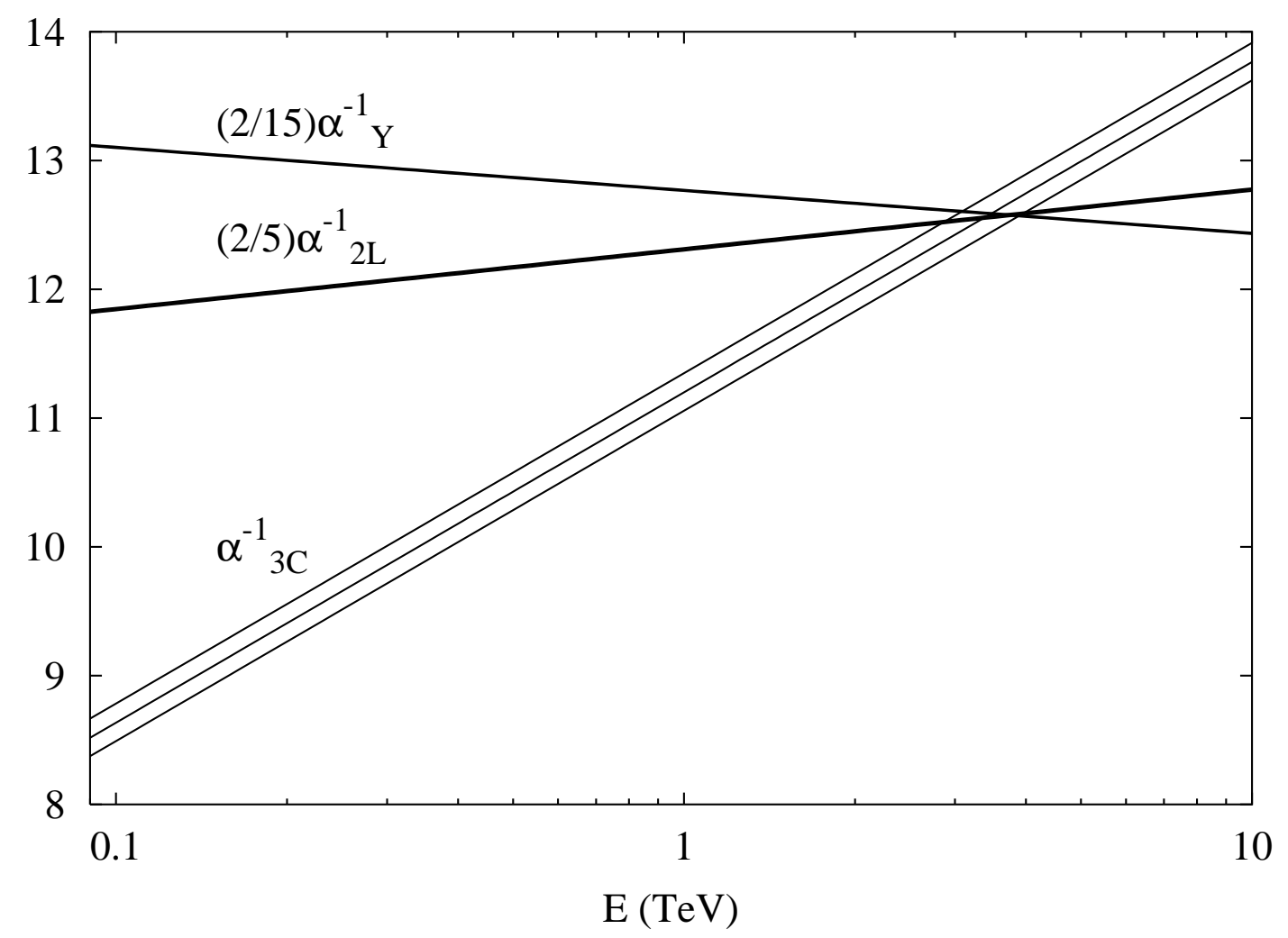

This grand unification works very precisely for the following bottom up reason. If we take the minimal standard model and run the couplings up in energy we find that the electroweak mixing angle $\sin ^{2} \theta_{W}(\mu)$ which has the value $\sim 0.231$ at scale $\mu=M_{Z}$ becomes $1 / 4$ at a scale $\mu \sim 4 \mathrm{TeV}$, actually nearer to 3.8 TeV. Another ratio $R(\mu)=\alpha_{3}(\mu) / \alpha_{2}(\mu)$ has a value larger than 3 at $\mu=M_{Z}$ then decreases through 3 at $400 \mathrm{GeV}$, becoming 2 at $140 \mathrm{TeV}$ then 1 at the GUT scale $\sim 10^{16} \mathrm{GeV}$. It goes through the simple rational fraction $R(\mu)=5 / 2$ at a value $\mu=3.8 \mathrm{TeV}$ within one percent of the scale where $\sin ^{2} \theta(\mu)=1 / 4$.

As a result the unification is perfect within errors as illustrated in the figure where all additional states are taken to be at $M_{U}=4 \mathrm{TeV}$. 


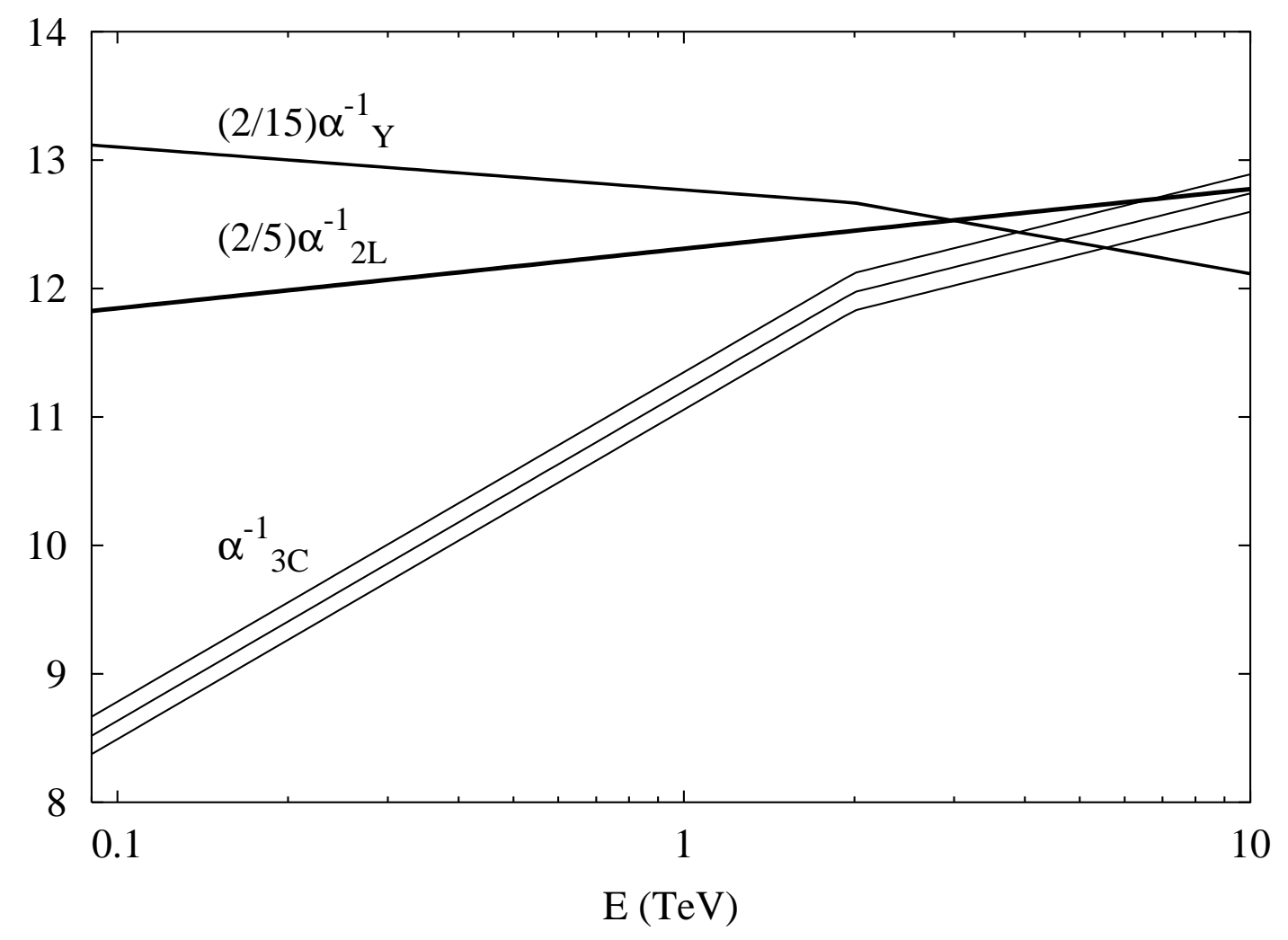

When the spectrum of additional states is altered there are threshold corrections which may spoil the perfect unification, as in more conventional grand unification. This example has " $\mathrm{CH}$ " fermions at $2 \mathrm{TeV}$, see [185]. 


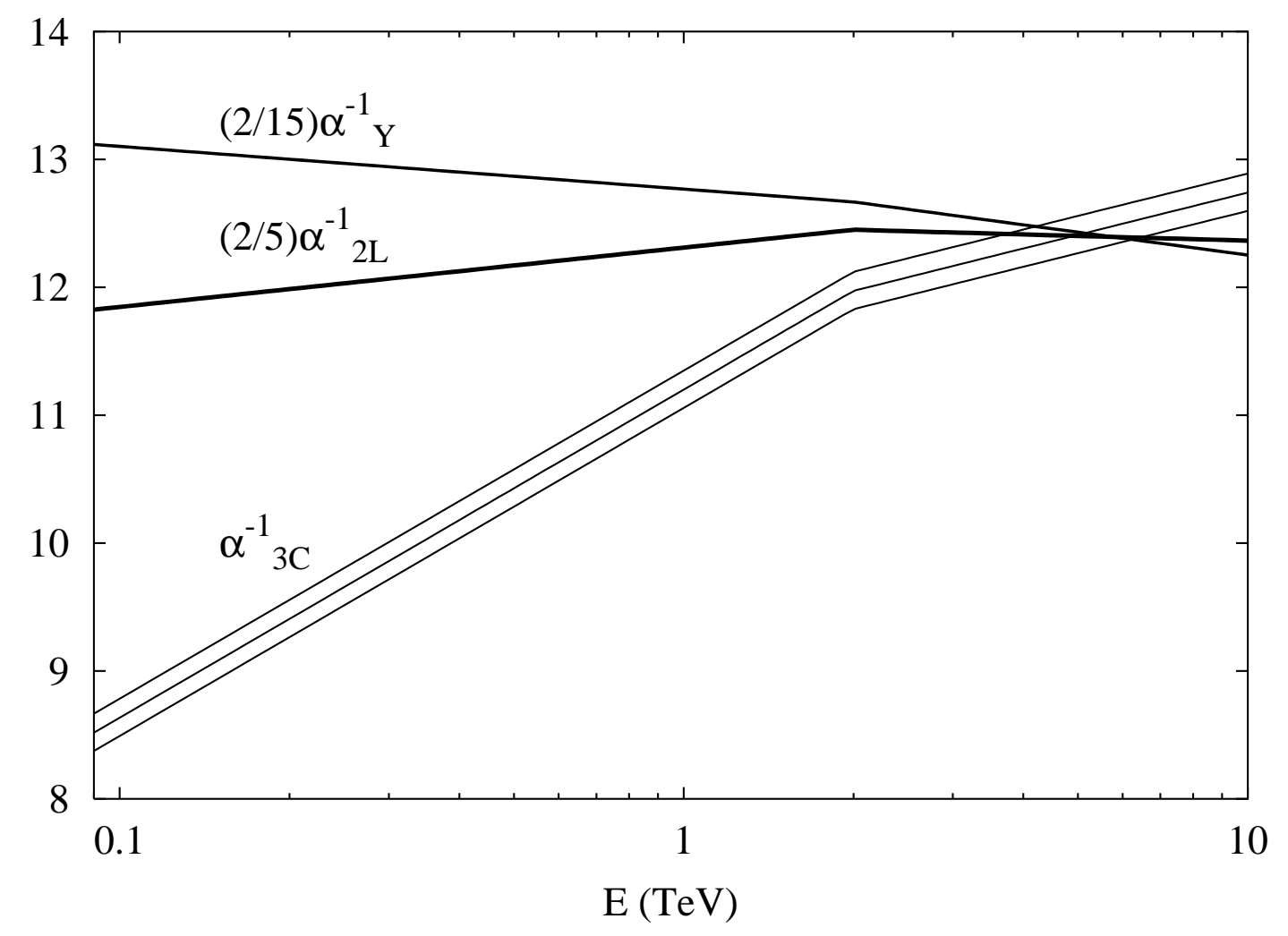

On the other hand, perfect unification can easily be maintained even with significant threshold corrections as indicated in this example which has "CW" fermions at $2 \mathrm{TeV}$, see [185]. 


\section{Quadratic divergences}

The lagrangian for the nonsupersymmetric $Z_{p}$ theory can be written in a convenient notation which accommodates simultaneously both adjoint and bifundamental scalars as

$$
\begin{aligned}
\mathcal{L}= & -\frac{1}{4} F_{\mu \nu ;, r, r}^{a b} F_{\mu \nu ;, r, r}^{b a}+i \bar{\lambda}_{r+A_{4}, r}^{a b} \gamma^{\mu} D_{\mu} \lambda_{r, r+A_{4}}^{b a}+2 D_{\mu} \Phi_{r+a_{i}, r}^{a b \dagger} D_{\mu} \Phi_{r, r+a_{i}}^{b a}+i \bar{\Psi}_{r+A_{m}, r}^{a b} \gamma^{\mu} D_{\mu} \Psi_{r, r+A_{m}}^{b a} \\
& -2 i g\left[\bar{\Psi}_{r, r+A_{i}}^{a b} P_{L} \lambda_{r+A_{i}, r+A_{i}+A_{4}}^{b c} \Phi_{r+A_{i}+A_{4}, r}^{\dagger c a}-\bar{\Psi}_{r, r+A_{i}}^{a b} P_{L} \Phi_{r+A_{i}, r-A_{4}}^{\dagger b c} \lambda_{r-A_{4}, r}^{c a}\right] \\
& -\sqrt{2} i g \epsilon_{i j k}\left[\bar{\Psi}_{r, r+A_{i}}^{a b} P_{L} \Psi_{r+A_{i}, r+A_{i}+A_{j}}^{b c} \Phi_{r-A_{k}-A_{4}, r}^{c a}-\bar{\Psi}_{r, r+A_{i}}^{a b} P_{L} \Phi_{r+A_{i}, r+A_{i}+A_{k}+A_{4}}^{b c} \Psi_{r-A_{j}, r}^{c a}\right] \\
& -g^{2}\left(\Phi_{r, r+a_{i}}^{a b} \Phi_{r+a_{i}, r}^{\dagger b c}-\Phi_{r, r-a_{i}}^{\dagger a b} \Phi_{r-a_{i}, r}^{b c}\right)\left(\Phi_{r, r+a_{j}}^{c c} \Phi_{r+a_{j}, r}^{\dagger d a}-\Phi_{r, r-a_{j}}^{\dagger c d} \Phi_{r-a_{j}, r}^{d a}\right) \\
& +4 g^{2}\left(\Phi_{r, r+a_{i}}^{a b} \Phi_{r+a_{i}, r+a_{i}+a_{j}}^{b c} \Phi_{r+a_{i}+a_{j}, r+a_{j}}^{\dagger c d} \Phi_{r+a_{j}, r}^{\dagger d a}-\Phi_{r, r+a_{i}}^{a b} \Phi_{r+a_{i}, r+a_{i}+a_{j}}^{b c} \Phi_{r+a_{i}+a_{j}, r+a_{i}}^{\dagger c d} \Phi_{r+a_{i}, r}^{\dagger d a}\right)
\end{aligned}
$$

where $\mu, \nu=0,1,2,3$ are lorentz indices; $a, b, c, d=1$ to $N$ are $U(N)^{p}$ group labels; $r=1$ to $p$ labels the node of the quiver diagram $; a_{i} \quad(i=\{1,2,3\})$ label the first three of the $\mathbf{6}$ of $\mathrm{SU}(4)$; $A_{m} \quad(m=\{1,2,3,4\})=\left(A_{i}, A_{4}\right)$ label the 4 of $\mathrm{SU}(4)$. By definition $A_{4}$ denotes an arbitrarily-chosen fermion $(\lambda)$ associated with the gauge boson, similarly to the notation in the $\mathcal{N}=1$ supersymmetric case.

Let us first consider the quadratic divergence question in the mother $\mathcal{N}=4$ theory. The $\mathcal{N}=4$ lagrangian is like Eq.(52) but since there is only one node all those subscripts become unnecessary so the form is simply

$$
\begin{aligned}
\mathcal{L}= & -\frac{1}{4} F_{\mu \nu}^{a b} F_{\mu \nu}^{b a}+i \bar{\lambda}^{a b} \gamma^{\mu} D_{\mu} \lambda^{b a}+2 D_{\mu} \Phi_{i}^{a b \dagger} D_{\mu} \Phi_{i}^{b a}+i \bar{\Psi}_{m}^{a b} \gamma^{\mu} D_{\mu} \Psi_{m}^{b a} \\
& -2 i g\left[\bar{\Psi}_{i}^{a b} P_{L} \lambda^{b c} \Phi_{i, r}^{\dagger c a}-\bar{\Psi}_{i}^{a b} P_{L} \Phi_{i}^{b c} \lambda^{c a}\right] \\
& -\sqrt{2} i g \epsilon_{i j k}\left[\bar{\Psi}_{i}^{a b} P_{L} \Psi_{j}^{b c} \Phi_{k}^{\dagger c a}-\bar{\Psi}_{i}^{a b} P_{L} \Phi_{j}^{b c} \Psi_{k}^{c a}\right] \\
& -g^{2}\left(\Phi_{i}^{a b} \Phi_{i}^{\dagger b c}-\Phi_{i}^{\dagger a b} \Phi_{i}^{b c}\right)\left(\Phi_{j}^{c d} \Phi_{j}^{\dagger d a}-\Phi_{j}^{\dagger c d} \Phi_{j}^{d a}\right) \\
& +4 g^{2}\left(\Phi_{i}^{a b} \Phi_{j}^{b c} \Phi_{i}^{\dagger c d} \Phi_{j}^{\dagger d a}-\Phi_{i}^{a b} \Phi_{j}^{b c} \Phi_{j}^{\dagger c d} \Phi_{i}^{\dagger d a}\right)
\end{aligned}
$$


All $\mathcal{N}=4$ scalars are in adjoints and the scalar propagator has one-loop quadratic divergences coming potentially from three scalar self-energy diagrams: (a) the gauge loop (one quartic vertex); (b) the fermion loop (two trilinear vertices); and (c) the scalar loop (one quartic vertex).

For $\mathcal{N}=4$ the respective contributions of $(\mathrm{a}, \mathrm{b}, \mathrm{c})$ are computable from Eq.(52) as proportional to $g^{2} N(1,-4,3)$ which cancel exactly.

The $\mathcal{N}=0$ results for the scalar self-energies $(\mathrm{a}, \mathrm{b}, \mathrm{c})$ are computable from the lagrangian of Eq.(52). Fortunately, the calculation was already done in the literature. The result is amazing! The quadratic divergences cancel if and only if $\mathrm{x}=3$, exactly the same "if and only if" as to have chiral fermions. It is pleasing that one can independently confirm the results directly from the interactions in Eq.(52) To give just one explicit example, in the contributions to diagram (c) from the last term in Eq.(52), the 1/N corrections arise from a contraction of $\Phi$ with $\Phi^{\dagger}$ when all the four color superscripts are distinct and there is consequently no sum over color in the loop. For this case, examination of the node subscripts then confirms proportionality to the kronecker delta, $\delta_{0, a_{i}}$. If and only if all $a_{i} \neq 0$, all the other terms in Eq.(52) do not lead to $1 / \mathrm{N}$ corrections to the $\mathcal{N}=4$.

We find it remarkable [216] and encouraging that the condition for naturalness, absence of one loop quadratic divergences, coincides with the necessary and sufficient condition for presence of chiral fermions. If the conditions had conflicted, as seemed $a$ priori possible, the approach would have had less phenomenological interest. 


\subsection{Is there a global symmetry?}

It is an old notion that $\mathcal{N}=4$ supersymmetric gauge theory is germane to the generalization of the standard model of particle phenomenology. The $\mathcal{N}=4$ theory has remarkable properties which include ultra violet finiteness and conformal invariance. Nevertheless, one striking feature of the standard model is the presence of chiral fermions which excludes both $\mathcal{N}=4$ and $\mathcal{N}=2$ extended supersymmetries. Also, although the situation might change, the absence of any experimental support even for $\mathcal{N}=1$ supersymmetry is striking.

Shortly after the discovery of supersymmetric theories, Haag, Lopuszanski and Sohnius generalized this to show in a 1975 publication [7] that under certain assumptions, supersymmetry is the only possibility.

No-go theorems can be useful because they provide a set of assumptions some or all of which must be violated in order to make progress. Here, we shall suggest [217] that the way around the generalized no-go theorem of Haag, Lopuszanki and Sohnius is to relax their assumption that the generators of the symmetry commute with gauge transformations. In particular, for a $U(N)^{n}$ quiver gauge theory, we suggest fermionic generators which transform as bi-bifundamentals under $U(N)^{n}$.

$\mathcal{N}=4$ supersymmetry

Here we collect briefly some well-known [218] facts, for convenience. 
The action for $\mathcal{N}=4$ Yang-Mills can be written

$$
\begin{aligned}
S= & \int d^{4} x\left[-\frac{1}{4} F_{\mu \nu a} F^{\mu \nu a}+\frac{1}{2} D_{\mu} \Phi_{i j}^{a} D^{\mu} \Phi_{i j}^{a}+i \bar{\chi}^{a} \gamma . D L \chi^{a}\right. \\
& -\frac{1}{2} i g f_{a b c}\left(\overline{\tilde{\chi}}^{a i} L \chi^{j b} \Phi_{i j}^{c}-\bar{\chi}_{i}^{a} R \tilde{\chi}_{j}^{b} \Phi^{i j c}\right) \\
& \left.-\frac{1}{4} g^{2}\left(f_{a b c} \Phi_{i j}^{b} \Phi_{k l}^{c}\right)\left(f_{a d e} \Phi^{i j d} \Phi^{k l e}\right)\right]
\end{aligned}
$$

where $\mu, \nu=0,1,2,3 ; i, j, k, l=1,2,3 ; L=\frac{1}{2}\left(1+\gamma_{5}\right), R=$ $\frac{1}{2}\left(1-\gamma_{5}\right)$; and $\tilde{\chi}_{i}=C \bar{\chi}^{i T}$ with $C$ the charge conjugation operator.

The action (52) is invariant under the $\mathcal{N}=4$ supersymmetry [218]

$$
\begin{aligned}
\delta A_{\mu}^{a} & =i\left(\bar{\alpha}_{i} \gamma_{\mu} L \chi^{i a}-\bar{\chi}_{i}^{a} \gamma_{\mu} L \alpha^{i}\right) . \\
\delta \Phi_{i j}^{a} & =i\left(\bar{\alpha}_{j} R \tilde{\chi}_{i}^{a}-\bar{\alpha}_{i} R \tilde{\chi}_{i}^{a}+\epsilon_{i j k l} \bar{\alpha}^{k} L \chi^{l a}\right) . \\
\delta L \chi^{i a} & =\sigma_{\mu \nu} F^{\mu \nu a} L \alpha^{i}-\gamma \cdot D \Phi^{i j a} R \tilde{\alpha}_{j}+\frac{1}{2} g f_{a b c} \phi_{b}^{i k} \Phi_{k j}^{c} L \alpha^{j} \\
\delta R \tilde{\chi}_{i}^{a} & =\sigma_{\mu \nu} F^{\mu \nu a} R \tilde{\alpha}_{i}+\gamma \cdot D \Phi_{i j}^{a} L \alpha^{i}+\frac{1}{2} g f_{a b c} \Phi_{i k}^{b} \Phi_{c}^{k j} L \tilde{\alpha}_{j} .
\end{aligned}
$$

where $\alpha^{i}$ transforms as a $\mathbf{4}$ and $\bar{\alpha}_{i}$ as a $\overline{\mathbf{4}}$ under an internal $S U(4)$ symmetry.

The group indices $a, b, c$ run over the dimension of the gauge group $a, b, c=1, \ldots ., d_{G}$. For $G=S U(N)$ or $U(N), d_{G}=$ $\left(N^{2}-1\right)$ or $N^{2}$ respectively. Note that the infintesimal supersymmetry parameter $\alpha^{i}$ is singlet under the gauge group $G$. This assumption will be relaxed for misaligned supersymmetry in the next section.

Misaligned supersymmetric gauge field theory (MSGFT)

The name is taken from [219] where string models without supersymmetry were studied, particularly the supertrace conditions necessary for cancellation of ultra violet divergences. 
The nonsupersymmetric quiver gauge theories here discussed satisfy such supertrace conditions if all scalars are in bifundamentals so the name "misaligned" supersymmetric gauge field theory (MSGFT) is appropriate. In [219], however, no explicit field transformation underlying misaligned supersymmetry was given and my aim here is to suggest how this may be accomplished.

A specific MSGFT model is defined by several integers, namely $N$ (the number of coalescing parallel D3 branes in AdS/CFT, also the $N$ in the gauge group $\left.U(N)^{n}\right), n$ (defining the abelian orbifold group $Z_{n}$, also the $n$ in the gauge group $\left.U(N)^{n}\right)$; and three integers $A_{1}, A_{2}, A_{3}$ which specify the embedding $Z_{n} \subset$ $S U(4)$ where $S U(4)$ is the internal symmetry of the $\mathcal{N}=4$ case corresponding to replacing the orbifold by a manifold. Note that the fourth integer $A_{4}$ defining the transformation of the 4 of $S U(4)$ is not independent because $A_{4}=-A_{1}-A_{2}-A_{3}(\mathrm{mod}$ $n)$. In summary, MSGFT models are specified by five integers $\left\{N, n, A_{1}, A_{2}, A_{3}\right\}$.

The action for such a MSGFT in the present notation

$$
\begin{aligned}
S= & \int d^{4} x\left[-\frac{1}{4} F_{\mu \nu a ; r, r} F_{r, r}^{\mu \nu a}+\frac{1}{2} D_{\mu} \Phi_{i j ; r+a_{i}, r}^{a} D^{\mu} \Phi_{i j ; r, r+a_{i}}^{a}+i \bar{\chi}_{r+A_{m}, r}^{a} \gamma . D L \chi_{r, r+A_{m}}^{a}\right. \\
& -\frac{1}{2} i g f_{a b c}\left(\bar{\chi}_{r, r+A_{m}}^{a i} L \chi_{r+A_{m}, r+A_{m}+A_{n}}^{j b} \Phi_{i j ; r+A_{m}+A_{n}, r}^{c}\right. \\
& \left.-\bar{\chi}_{i ; r, r+A_{m}}^{a} R \tilde{\chi}_{j ; r+A_{m}, r-A_{n}}^{b} \Phi_{r-A_{n}, r}^{i j c}\right) \\
& \left.-\frac{1}{4} g^{2}\left(f_{a b c} \Phi_{i j ; r, r+a_{i}}^{b} \Phi_{k l ; r+a_{i}, r+a_{i}+a_{j}}^{c}\right)\left(f_{a d e} \Phi_{r+a_{i}+a_{j}, r+a_{j}}^{i j d} \Phi_{r+a_{j}, r}^{k l e}\right)\right]
\end{aligned}
$$

in which the $a_{i}$ are defined by $a_{i}=A_{2}+A_{3}, a_{2}=A_{3}+A_{1}, a_{3}=$ $A_{1}+A_{2}$; the subscript $r=1,2, \ldots n$ is a node label; when the two node superscripts are equal it is an adjoint plus singlet of that $U(N)_{r}$; when the two subscripts are unequal it is a bifundamental and the two gauge labels transform under different $U(N)$ gauge groups. 
Now we address the question of what variation of the fields in the action (54) will leave it invariant. Given the field content, the infinitesimal fermionic parameters must transform under the $U(N)^{n}$ gauge group. As a generalization of equations (53), we suggest

$$
\begin{aligned}
& \delta\left(A_{\mu}^{(p)}\right)_{\alpha_{p}}^{\beta_{p}} \\
& =i\left(\left[\bar{\alpha}_{i}\right]_{\alpha_{p}, \delta_{p+A_{m}}}^{\beta_{p}, \gamma_{p}} \gamma_{\mu} L\left(\chi^{i\left(p, p+A_{m}\right)}\right)_{\gamma_{p}}^{\delta_{p+A_{m}}}-\left(\bar{\chi}_{i}^{\left(p-A_{m}, p\right)}\right)_{\gamma_{p-A_{m}}}^{\delta_{p}} \gamma_{\mu} L\left[\alpha^{i}\right]_{\alpha_{p}, \delta_{p}}^{\alpha_{p}, \gamma_{p-A_{m}}}\right) \\
& \delta\left(\Phi_{i j}^{\left(p, p+a_{i}\right)}\right)_{\alpha_{p}}^{\alpha_{p+a_{i}}} \\
& =i\left(\left[\bar{\alpha}_{j}\right]_{\alpha_{p}, \beta_{p+A_{m}}}^{\alpha_{p+a_{i}, \beta_{p}}} R\left(\tilde{\chi}_{i}^{\left(p, p+A_{m}\right)}\right)_{\beta_{p}}^{\beta_{p+A_{m}}}-\left[\bar{\alpha}_{i}\right]_{\alpha_{p}, \beta_{p+A_{m}}}^{\alpha_{p+a_{i}, \beta_{p}}} R\left(\tilde{\chi}_{j}^{\left(p, p+A_{m}\right)}\right)_{\beta_{p}}^{\beta_{p+A_{m}}}\right. \\
& \left.+\epsilon_{i j k l}\left[\bar{\alpha}^{k}\right]_{\alpha_{p}, \beta_{p+A_{m}}}^{\alpha_{p+a_{i}, \beta_{p}}} L\left(\chi^{l\left(p, p+A_{m}\right)}\right)_{\beta_{p}}^{\beta_{p+A_{m}}}\right) . \\
& \delta\left(L \chi^{i\left(p, p+A_{m}\right)}\right)_{\alpha_{p}}^{\alpha_{p+A_{m}}} \\
& =\sigma_{\mu \nu}\left(F^{\mu \nu(p)}\right)_{\beta_{p}}^{\gamma_{p}} L\left[\alpha^{i}\right]_{\alpha_{p} \gamma_{p}}^{\alpha_{p+A_{m}} \beta_{p}} \\
& -\gamma \cdot D\left(\Phi^{i j\left(p, p+a_{i}\right)}\right)_{\beta_{p}}^{\beta_{p+a_{i}}} R\left[\tilde{\alpha}_{j}\right]_{\alpha_{p} \beta_{p+a_{i}}}^{\alpha_{p+A_{m}} \beta_{p}} \\
& +\frac{1}{2} g \epsilon_{\alpha_{p+a_{i}} \beta_{p+a_{i}} \gamma_{p+a_{i}}} \epsilon^{\beta_{p} \gamma_{p} \delta_{p}}\left(\phi^{i k\left(p, p+a_{i}\right)}\right)_{\gamma_{p}}^{\beta_{p+a_{i}}}\left(\Phi_{k j}^{\left(p, p+a_{i}\right)}\right)_{\delta_{p}}^{\gamma_{p+a_{i}}} L\left[\alpha^{j}\right]_{\alpha_{p} \beta_{p}}^{\alpha_{p+A_{m}} \alpha_{p+a_{i}}} \\
& \delta\left(R \tilde{\chi}_{i}^{\left(p-A_{m}, p\right)}\right)_{\alpha_{p-A_{m}}}^{\alpha_{p}} \\
& =\sigma_{\mu \nu}\left(F^{\mu \nu(p)}\right)_{\beta_{p}}^{\gamma_{p}} R\left[\tilde{\alpha}_{i}\right]_{\alpha_{p-A_{m}} \gamma_{p}}^{\alpha_{p} \beta_{p}} \\
& +\gamma \cdot D\left(\Phi_{i j}^{\left(p-a_{i}, p\right)}\right)_{\beta_{p-a_{i}}}^{\beta_{p}} L\left[\alpha^{j}\right]_{\alpha_{p-A_{m}} \beta_{p}}^{\alpha_{p} \beta_{p-a_{i}}} \\
& +\frac{1}{2} g \epsilon_{\alpha_{p+a_{i}} \beta_{p+a_{i}} \gamma_{p+a_{i}}} \epsilon^{\beta_{p} \gamma_{p} \delta_{p}}\left(\phi_{i k}^{\left(p, p+a_{i}\right)}\right)_{\gamma_{p}}^{\beta_{p+a_{i}}}\left(\Phi^{k j\left(p, p+a_{i}\right)}\right)_{\delta_{p}}^{\gamma_{p+a_{i}}} R\left[\tilde{\alpha}_{j}\right]_{\alpha_{p-A_{m} \beta_{p}} \beta_{p} \alpha_{p+a_{i}}}^{\alpha_{1}}(55)
\end{aligned}
$$

The equations (55) are written so that they reduce to the $\mathcal{N}=$ 4 equations (53) when the internal $U(N)^{n}$ dependence of the fermionic generators is removed and are written such that the transformation properties under the gauge group $U(N)^{n}$ are consistent for each term in the field transformations (55)).

In the limit $A_{m}=a_{i}=0$ and $n=1$, the bifundamentals become adjoints and the couplings in the transformations Eq.(555) 
reduce to those in Eq.(53); this requirement excludes further (symmetric) cubic couplings in Eq. (55).

We see that the infinitesimal generators $\mathrm{n}$ Eq. (55) must generically be outer products of two bifundamentals under $U(N)^{n}$ although in all terms of (55) this reduces to an outer product of one adjoint with one bifundamental. In the transformation of the $\chi^{i}$ fields I have for definiteness specialized to the case $N=3$ in generalizing the structure constants $f_{a b c}$ of (53) for adjoint representations to the antisymmetric tensors $\epsilon_{\alpha \beta \gamma}$ in (55) for bifundamental representations; for general $N$ one can form a unique antisymmetric cubic invariant from bifundamentals writable in two equivalent forms

$$
f_{a b c}\left(\lambda^{a}\right)_{i^{\prime}}^{i}\left(\lambda^{b}\right)_{j^{\prime}}^{j}\left(\lambda^{c}\right)_{k^{\prime}}^{k} \Phi_{i}^{i^{\prime}} \Phi_{j}^{j^{\prime}} \Phi_{k}^{k^{\prime}} \quad \text { or } \quad \epsilon^{i j k l m n \ldots x y z} \epsilon_{i^{\prime} j^{\prime} k^{\prime} l m n \ldots x y z} \Phi_{i}^{i^{\prime}} \Phi_{j}^{j^{\prime}} \Phi_{k}^{k^{\prime}}
$$

\section{Discussion.}

A first issue concerns the no-go theorems of [7]. There is no problem with the earlier paper which did not consider fermionic generators and the generalized no-go theorem implicitly assumes that the fermionic generators are singlets under the gauge group; since this assumption is violated in misaligned supersymmetry, the no-go theorem is inapplicable.

There remain a number of questions to be explored: Does variation [217] under the field transformations (55) really provide an exact symmetry of the action (54)? Do the generators form a closed algebra and the transformations a group? What are the representations of this group? The quiver diagram must form a representation but it may be reducible. It would be interesting to know the irreducible representations. Do MSGFT share properties of supersymmetric gauge theories such as non renormalization theorems? Can a MSGFT be conformally invariant? 


\section{$9 \quad \mathrm{U}(1)$ factors}

The lagrangian for the nonsupersymmetric $Z_{n}$ theory can be written in a convenient notation which accommodates simultaneously both adjoint and bifundamental scalars as mentioned before.

As also mentioned above we shall restrict attention to models where all scalars are in bifundamentals which requires all $a_{i}$ to be non zero. Recall that $a_{1}=A_{2}+A_{3}, a_{2}=A_{3}+A_{1} ; a_{3}=A_{1}+A_{2}$.

The lagrangian is classically $U(N)^{p}$ gauge invariant. There are, however, triangle anomalies of the $U(1)_{p} U(1)_{q}^{2}$ and $U(1)_{p} S U(N)_{q}^{2}$ types. Making gauge transformations under the $U(1)_{r}(\mathrm{r}=$ $1,2, \ldots, \mathrm{n})$ with gauge parameters $\Lambda_{r}$ leads to a variation

$$
\delta \mathcal{L}=-\frac{g^{2}}{4 \pi^{2}} \Sigma_{p=1}^{p=n} A_{p q} F_{\mu \nu}^{(p)} \tilde{F}^{(p) \mu \nu} \Lambda_{q}
$$

which defines an $n \times n$ matrix $A_{p q}$ which is given by

$$
A_{p q}=\operatorname{Tr}\left(Q_{p} Q_{q}^{2}\right)
$$

where the trace is over all chiral fermion links and $Q_{r}$ is the charge of the bifundamental under $U(1)_{r}$. We shall adopt the sign convention that $\mathbf{N}$ has $Q=+1$ and $\mathbf{N}^{*}$ has $Q=-1$.

It is straightforward to write $A_{p q}$ in terms of Kronecker deltas because the content of chiral fermions is

$$
\sum_{m=1}^{m=4} \Sigma_{r=1}^{r=n}\left(\mathbf{N}_{r}, \mathbf{N}^{*}{ }_{r+A_{m}}\right)
$$

This implies that the antisymmetric matrix $A_{p q}$ is explicitly 


$$
A_{p q}=-A_{q p}=\Sigma_{m=1}^{m=4}\left(\delta_{p, q-A_{m}}-\delta_{p, q+A_{m}}\right)
$$

Now we are ready to construct $\mathcal{L}_{\text {comp }}^{(1)}$, the compensatory term [112]. Under the $U(1)_{r}$ gauge transformations with gauge parameters $\Lambda_{r}$ we require that

$$
\begin{aligned}
\delta \mathcal{L}_{\text {comp }}^{(1)} & =-\delta \mathcal{L} \\
& =+\frac{g^{2}}{4 \pi^{2}} \Sigma_{p=1}^{p=n} A_{p q} F_{\mu \nu}^{(p)} \tilde{F}^{(p) \mu \nu} \Lambda_{q}
\end{aligned}
$$

To accomplish this property, we construct a compensatory term in the form

$$
\mathcal{L}_{\text {comp }}^{(1)}=\frac{g^{2}}{4 \pi} \sum_{p=1}^{p=n} \Sigma_{k} B_{p k} \operatorname{Im} \operatorname{Tr} \ln \left(\frac{\Phi_{k}}{v}\right) F_{\mu \nu}^{(p)} \tilde{F}^{(p) \mu \nu}
$$

where $\Sigma_{k}$ runs over scalar links. To see that $\mathcal{L}_{\text {comr }}^{(1)}$ of Eq.(62) has $S U(N)^{n}$. invariance rewrite $\operatorname{Tr} \ln \equiv \exp \operatorname{det}$ and note that the $S U(N)$ matrices have unit determinant.

We note en passant that one cannot take the $v \rightarrow 0$ limit in Eq. (62); the chiral anomaly enforces a breaking of conformal invariance.

We define a matrix $C_{k q}$ by

$$
\delta\left(\Sigma_{p=1}^{p=n} \Sigma_{k} \operatorname{Im} \operatorname{Tr} \ln \left(\frac{\Phi_{k}}{v}\right)\right)=\Sigma_{q=1}^{q=n} C_{k q} \Lambda_{q}
$$

whereupon Eq.(61) will be satisfied if the matrix $B_{p k}$ satisfies $A=B C$. The inversion $B=A C^{-1}$ is non trivial because $C$ is singular but $C_{k q}$ can be written in terms of Kronecker deltas by noting that the content of complex scalar fields in the model implies that the matrix $C_{k q}$ must be of the form

$$
C_{k q}=3 \delta_{k q}-\Sigma_{i} \delta_{k+a_{i}, q}
$$




\subsection{Conformality of $\mathrm{U}(1)$ couplings.}

$U(1)$ factors are a major concern in quiver gauge theories. In the absence of the compensatory term, the two independent $U(N)^{n}$ gauge couplings $g_{N}$ for $\mathrm{SU}(\mathrm{N})$ and $g_{1}$ for $\mathrm{U}(1)$ are taken to be equal $g_{N}\left(\mu_{0}\right)=g_{1}\left(\mu_{0}\right)$ at a chosen scale, e.g. $\mu_{0}=4 \mathrm{TeV}$ to enable cancellation of quadratic divergences. Note that the $n \mathrm{SU}(\mathrm{N})$ couplings $g_{N}^{(p)}$ are equal by the overall $Z_{n}$ symmetry, as are the $n \mathrm{U}(1)$ couplings $g_{1}^{(p)}, 1 \leq p \leq n$.

As one evolves to higher scales $\mu>\mu_{0}$, the renormalization group beta function $\beta_{N}$ for $\mathrm{SU}(\mathrm{N})$ vanishes $\beta_{N}=0$ at least at one-loop level so the $g_{N}(\mu)$ can behave independent of the scale as expected by conformality. On the other hand, the beta function $\beta_{1}$ for $\mathrm{U}(1)$ is positive definite in the unadorned theory, given at one loop by

$$
b_{1}=\frac{11 N}{48 \pi^{2}}
$$

where $\mathrm{N}$ is the number of colors.

The corresponding coupling satisfies

$$
\frac{1}{\alpha_{1}(\mu)}=\frac{1}{\alpha_{1}(M)}+8 \pi b_{1} \ln \left(\frac{M}{\mu}\right)
$$

so the Landau pole, putting $\alpha(\mu)=0.1$ and $N=3$, occurs at

$$
\frac{M}{\mu}=\exp \left[\frac{20 \pi}{11}\right] \simeq 302
$$

so for $\mu=4 \mathrm{TeV}, M \sim 1200 \mathrm{TeV}$. The coupling becomes "strong" $\alpha(\mu)=1$ at

$$
\frac{M}{\mu}=\exp \left[\frac{18 \pi}{11}\right] \simeq 171
$$

or $M \sim 680 \mathrm{TeV}$. 
We may therefore ask whether the new term $\mathcal{L}_{\text {comp }}$ in the lagrangian, necessary for anomaly cancellation, can solve [112] this problem for conformality? 
Since the scale $v$ breaks conformal invariance, the matter fields acquire mass, so the one-loop diagram \#4 has a logarithmic divergence proportional to

$$
\int \frac{d^{4} p}{v^{2}}\left[\frac{1}{\left(p^{2}-m_{k}^{2}\right)}-\frac{1}{\left(p^{2}-m_{k^{\prime}}^{2}\right)}\right] \sim-\frac{\Delta m_{k k^{\prime}}^{2}}{v^{2}} \ln \left(\frac{\Lambda}{v}\right)
$$

the sign of which depends on $\delta m_{k k^{\prime}}^{2}=\left(m_{k}^{2}-m_{k^{\prime}}^{2}\right)$.

To achieve conformality of $\mathrm{U}(1)$, a constraint must be imposed on the mass spectrum of matter bifundamentals, viz

$$
\Delta m_{k k^{\prime}}^{2} \propto v^{2}\left(\frac{11 N}{48 \pi^{2}}\right)
$$

with a proportionality constant of order one which depends on the choice of model, the $n$ of $Z_{n}$ and the values chosen for $A_{m}, m=1,2,3$. This signals how conformal invariance must be broken at the $\mathrm{TeV}$ scale in order that it can be restored at high energy; it is interesting that such a constraint arises in connection with an anomaly cancellation mechanism which necessarily breaks conformal symmetry.

\footnotetext{
\#4 The usual one-loop $\beta$-function is of order $h^{2}$ regarded as an expansion in Planck's constant: four propagators each $\sim h$ and two vertices each $\sim h^{-1}$ (c.f. [220]). The diagram considered is also $\sim h^{2}$ since it has three propagators, one quantum vertex $\sim h$ and an additional $h^{-2}$ associated with $\Delta m_{k k^{\prime}}^{2}$.
} 


\section{Dark matter candidate}

In the nonsupersymmetric quiver gauge theories, the gauge group, for abelian orbifold $A d S_{5} \times S^{5} / Z_{n}$ is $U(N)^{n}$. In phenomenological application $N=3$ and $n$ reduces eventually after symmetry breaking to $n=3$ as in trinification. The chiral fermions are then in the representation of $S U(3)^{3}$ :

$$
\left(3,3^{*}, 1\right)+\left(3^{*}, 1,3\right)+\left(1,3,3^{*}\right)
$$

This is as in the $\mathbf{2 7}$ of $E_{6}$ where the particles break down in to the following representations of the $S U(3) \times S U(2) \times U(1)$ standard model group:

$$
Q, \quad u^{c}, \quad d^{c}, \quad L \quad e^{c} \quad N^{c}
$$

transforming as

$$
(3,2), \quad\left(3^{*}, 1\right), \quad\left(3^{*}, 1\right), \quad(1,2), \quad(1,1), \quad(1,1)
$$

in a $\mathbf{1 6}$ of the $S O(10)$ subgroup.

In addition there are the states

$$
h, \quad h *, \quad E, \quad E *
$$

transforming as

$$
(3,1), \quad\left(3^{*}, 1\right), \quad(2,1), \quad(2,1)
$$

in a 10 of $S O(10)$ and finally

transforming as the singlet

$$
(1,1)
$$

It is natural to define [221] a $Z_{2}$ symmetry $R$ which commutes with the $S O(10)$ subgroup of $E_{6} \rightarrow O(10) \times U(1)$ 
It is natural to define a $Z_{2}$ symmetry $R$ which commutes with the $S O(10)$ subgroup of $E_{6} \rightarrow O(10) \times U(1)$ such that $R=+1$ for the first 16 of states. Then it is mandated that $R=-1$ for the $\mathbf{1 0}$ and 1 of SO(10) because the following Yukawa couplings must be present to generate mass for the fermions:

$$
16_{f} 16_{f} 10_{s}, \quad 16_{f} 10_{f} 16_{s}, \quad 10_{f} 10_{f} 1_{s}, \quad 10_{f} 1_{f} 1_{s}
$$

which require $R=+1$ for $10_{s}, 1_{s}$ and $R=-1$ for $16_{s}$.

Contribution of the Lightest Conformality Particle (LCP) to the cosmological energy density:

The LCP act as cold dark matter WIMPs, and the calculation of the resultant energy density follows a well-known path. Here we follow the procedure in a recent technical book by Mukhanov.

The LCP decouple at temperature $T_{*}$, considerably less than their mass $M_{L C P}$; we define $x_{*}=M_{L C P} / T_{*}$. Let the annihilation cross-section of the LCP at decoupling be $\sigma_{*}$. Then the dark matter density $\Omega_{m}$, relative to the critical density,

$$
\Omega_{m} h_{75}^{2}=\frac{\tilde{g}_{*}^{1 / 2}}{g_{*}} x_{*}^{3 / 2}\left(\frac{3 \times 10^{-38} \mathrm{~cm}^{2}}{\sigma_{*}}\right)
$$

where $h_{75}$ is the Hubble constant in units of $75 \mathrm{~km} / \mathrm{s} / M p c . g_{*}=$ $\left(g_{b}+\frac{7}{8} g_{f}\right)$ is the effective number of degrees of freedom (dof) at freeze-out for all particles which later convert their energy into photons; and $\tilde{g}_{*}$ is the number of dof which are relativistic at $T_{*}$.

The LCP [221] is a viable candidate for a cold dark matter particle which can be produced at the LHC. The distinction from other candidates will require establishment of the $U(3)^{3}$ gauge bosons, extending the 3-2-1 standard model and the discovery that the LCP is in a bifundamental representation thereof. To confirm that the LCP is the dark matter particle would, however, require direct detection of dark matter. 


\section{Proton decay}

Here we address the issue of proton decay. Under $S U(3)_{C} \times$ $S U(3) \times S U(3)$, the quarks and leptons families each appear as in trinification [163] in the representations

$$
\left[\left(3,3^{*}, 1\right)+\left(1,3,3^{*}\right)+\left(3^{*}, 1,3\right)\right]
$$

for which the baryon numbers are respectively $B=+1 / 3,0,-1 / 3$ for quarks, leptons and antiquarks.

The gauge bosons cannot transform quarks into leptons or vice versa because of the factoring out of the color $S U(3)_{C}$ group. So unlike in $S U(5)([222,223])$ proton decay is absent in the gauge sector.

The scalars are likewise in 27's according to

$$
\left[\left(3,3^{*}, 1\right)+\left(1,3,3^{*}\right)+\left(3^{*}, 1,3\right)\right]+\text { c.c. }
$$

although here, unlike for the fermions the complex conjugate representations must be included.

Fermion masses arise in trinification [163] from Yukawa couplings of the form

$$
\left(3,3^{*}, 1\right)_{q}\left(3^{*}, 1,3\right)_{q}\left(1,3,3^{*}\right)_{\phi}
$$

for the quarks and

$$
\left(1,3,3^{*}\right)_{f}\left(1,3,3^{*}\right)_{f}\left(1,3,3^{*}\right)_{\phi}
$$

for the leptons. Because these are two independent couplings, trinification has the feature of giving no relationship between quark and lepton masses.

There are additional Yukawa couplings possible which would violate baryon number $B$ and cause catastrophically rapid proton decay with a $\mathrm{TeV}$ unification scale. Therefore these must be forbidden, as is achieved [224] by assigning a generalization 
of baryon number $B=+1 / 3,0-1 / 3$ respectively to the three representation listed.

$$
\begin{array}{cc}
\left(3,3^{*}, 1\right)_{\phi} & B=1 / 3 \\
\left(1,3,3^{*}\right)_{\phi} & B=0 \\
\left(3^{*}, 1,3\right)_{\phi} & B=-1 / 3
\end{array}
$$

Such assignmnents are very natural.

In phenomenological analysis of trinification [215], however, such a procedure is avoided in order to be able to obtain acceptable quark and lepton mass matrices. With teravolt unification such departure from the simplest case is not possible as proton decay must be totally forbidden.

However, the quark and lepton masses in conformality will acquire contributions in relevant operators $m_{i} \psi \psi$ from breaking of conformal symmetry. The pattern of these masses cannot yet be calculated, although a constraint on the pattern of the corresponding scalar masses has been suggested in [216] based on the cancellation of quadratic divergences.

In grand unification based on conformality, therefore, it is expected that proton decay will be completely absent (See however [225].) in the non-gravitational theory. Gravitational effects may eventually destabilize the proton but with lifetime $\sim 10^{50} y$ far beyond any forseeable experiment.

Equally or more important is the realization that consistency of the conformality with proton decay dictates that the quark and lepton masses receive significant contributions from the four-dimensional conformal symmetry breaking, not only from the Yukawa coupling to Higgs as universally assumed in previous studies of grand unification.

In the standard model, while the $Z^{0}, W^{ \pm}$masses are accurately predicted by a Higgs mechanism, there is no similar statement about fermion masses. The conclusion is that quark and 
lepton masses arise principally as relevant operators \#5 arising from breaking of four-dimensional conformal invariance; a corollary is that the couplings of the standard Higgs scalar to quarks and leptons depart from the values usually assumed.

\#5 One important question is how such mass terms are induced above the electroweak scale. One possibility is via four fermion operators. 


\section{Conclusions}

It has been established that conformality can provide (i) naturalness without one-loop quadratic divergence for the scalar mass and anomaly cancellation; (ii) precise unification of the coupling constants; and (iii) a viable dark matter candidate. It remains for experiment to check that quiver gauge theories with gauge group $U(3)^{3}$ or $U(3)^{n}$ with $n \geq 4$ are actually employed by Nature.

For completeness, we should note that possible problems with $\mathcal{N}=0$ orbifolds have been pointed out both in one-loop calculations in field theory [226] and from studies of tachyonic instability in the ancestral string theory [227].

One technical point worth another mention is that while the $U(1)$ anomalies discussed in Section 9 are cancelled in sting theory, in the gauge theory we have shown a different description of such cancellation which has the advantage of suggesting how $U(1)$ gauge couplings may be conformally invariant at high energies. This is important because in string theory, except for special linear combinations, all such $U(1)$ factors acquire mass by the Stückelberg mechanism while in the gauge field theory as discussed in Section 8 the cancellation of quadratic divergences and solution of the hierarchy problem for $\mathcal{N}=0$ require they be instead at the teravolt scale.

We have described how phenomenology of conformality has striking resonances with the standard model, as we have described optimistically as experimental evidence in its favor. We have described how $4 \mathrm{TeV}$ Unification predicts three families and new particles around $4 \mathrm{TeV}$ accessible to experiment (LHC).

It is encouraging that the scalar propagator in these theories has no quadratic divergence if and only if there are chiral fermions. Anomaly cancellation in the effective lagrangian has been tied to the conformality of $\mathrm{U}(1)$ gauge couplings.

A dark matter candidate $(\mathrm{LCP}=$ Lightest Conformality Par- 
ticle) may be produced at the Large Hadron Collider (LHC), then directly detected from the cosmos. Study of proton decay leads to the conclusion that quark and lepton masses arise not from the Yukawa couplings of the standard model but from operators induced in breaking of the four-dimensional conformal invariance. This implies that the Higgs couplings differ from those usually assumed in the unadorned standard model. This is yet another prediction from conformality to be tested when the Higgs scalar couplings and decay products are examined at the LHC in the near future. 


\section{Acknowledgements}

TWK thanks the Aspen Center for Physics for hospitality while this work was in progress. This work was supported in part by the U.S. Department of Energy under Grants No. DEFG02-06ER41418 (PHF) and DE-FG05-85ER40226 (TWK). 


\section{Note Added}

When the speculation was originally made in [14] that non supersymmetric $\mathcal{N}=0$ abelian orbifolded theories with gauge group $U(N)^{n}$ become conformally invariant (conformality) at high energy for finite $N$ it was believed that in the limit $N \rightarrow \infty$ conformality is correct, as suggested by the papers [228, 229] which provided a "proof" that in the large $\mathrm{N}$ limit the $\mathcal{N}=0$ theory coincided to all orders of perturbation theory with the ancestor $\mathcal{N}=4$ theory which is known to be conformally invariant.

For $\mathcal{N}=4$ gauge theory conformality is known [10-12] not only for the limit $N \rightarrow \infty$ but also for finite $N$. It was therefore natural in [14] to conjecture the same for $\mathcal{N} \leq 2$ including for the nonsupersymmetric case $\mathcal{N}=0$.

More recent work by Dymarsky, Klebanov and Roiban [230,231] shows that the "proof" in $[228,229]$ is false because there are double trace operators of the bifundamental scalars with the general form $\operatorname{Tr}(\Phi \Phi) \operatorname{Tr}(\Phi \Phi)$ which are forbidden by $\mathcal{N}=4$ symmetry from appearing in the ancestor theory but which can and do appear as counterterms in the $\mathcal{N}=0$ theory. The renormalization group $\beta$-functions for these double trace couplings do not vanish generically in the $N \rightarrow \infty$ limit so the conformality of [14] requires more than cancellation of $1 / N$ corrections.

The important papers $[230,231]$ study this phenomenon at the one-loop level. The impact on the all orders speculation of [14] about $\mathcal{N}=0$ and finite $N$ merits further investigation. 


\section{References}

[1] K.G. Wilson, Phys. Rev. D3, 1818 (1971).

[2] K.G. Wilson, Nucl. Phys. Proc. Suppl. 140, 3 (2005). hep-lat/0412043.

[3] S.L. Glashow, Nucl. Phys. 22, 579 (1961).

[4] S. Weinberg, Phys. Rev. Lett. 19, 1264 (1967).

[5] A. Salam, in Svartholm: Elementary Particle Theory, Proceedings of the Nobel Symposium held 1968 at Lerum, Sweden, Stockholm (1968) page 367-377.

[6] J. Wess and B. Zumino, Nucl. Phys. B70, 39 (1974).

[7] R. Haag, J.T. Lopuszansi and M. Sohnius, Nucl. Phys. B88, 257 (1975).

[8] S. Coleman and J.E. Mandula, Phys. Rev. 159, 1251 (1967).

[9] P. Woit, Not Even Wrong. Basic Books (2006).

[10] S. Mandelstam, Nucl. Phys. B213, 149 (1983).

[11] P. Howe and K. Stelle, Phys. Lett. B137 135 (1984);

[12] L. Brink, Talk at Johns Hopkins Workshop on Current Problems in High Energy Particle Theory, Bad Honnef, Germany (1983).

[13] J.M. Maldacena, Adv. Theor. Math. Phys. 2, 231 (1998). hep-th/9711200.

[14] P.H. Frampton, Phys.Rev. D60, 041901 (1999). hep-th/9812117.

[15] F. D. Smith, hep-th/9306011.

[16] M. R. Douglas and G. W. Moore, hep-th/9603167. 
[17] A. V. Sardo-Infirri, alg-geom/9610005.

[18] J. de Boer, K. Hori, H. Ooguri and Y. Oz, Nucl. Phys. B 493 (1997) 101 hep-th/9611063.

[19] F. Xu, q-alg/9707003.

[20] K. Okuyama and Y. Sugawara, JHEP 9808 (1998) 002 hep-th/9806001.

[21] A. Kapustin, JHEP 9812 (1998) 015 hep-th/9806238.

[22] Y. Sugawara, JHEP 9906 (1999) 035 hep-th/9903120.

[23] B. Feng, A. Hanany and Y. H. He, hep-th/9909125.

[24] Y. H. He, hep-th/9911114.

[25] M. Buican, D. Malyshev, D. R. Morrison, M. Wijnholt and H. Verlinde, JHEP 0701 (2007) 107 hep-th/0610007.

[26] H. Verlinde and M. Wijnholt, JHEP 0701 (2007) 106 hep-th/0508089.

[27] B. Fiol and M. Marino, JHEP 0007 (2000) 031 hep-th/0006189.

[28] A. M. Uranga, hep-th/0007173.

[29] C. Albertsson, B. Brinne, U. Lindstrom, M. Rocek and R. von Unge, Nucl. Phys. Proc. Suppl. 102 (2001) 3 hep-th/0103084.

[30] S. Govindarajan and T. Jayaraman, Nucl. Phys. B 600 (2001) 457 hep-th/0010196.

[31] B. Feng, A. Hanany, Y. H. He and N. Prezas, JHEP 0104 (2001) 037 hep-th/0011192.

[32] D. Berenstein, V. Jejjala and R. G. Leigh, Phys. Rev. D 64 (2001) 046011 hep-th/0012050. 
[33] C. Albertsson, B. Brinne, U. Lindstrom and R. von Unge, JHEP 0105 (2001) 021 hep-th/0102038.

[34] T. W. Kephart and Q. Shafi, Phys. Lett. B520, 313 (2001). hep-ph/0105237.

[35] T. Muto and T. Tani, JHEP 0109 (2001) 008 hep-th/0107217.

[36] F. Cachazo, S. Katz and C. Vafa, hep-th/0108120.

[37] A. Hanany and A. Iqbal, JHEP 0204 (2002) 009 hep-th/0108137.

[38] L. Alvarez-Consul and O. Garcia-Prada, arXiv:math.dg/0112160.

[39] L. Alvarez-Consul and O. Garcia-Prada, Commun. Math. Phys. 238 (2003) 1 arXiv:math.dg/0112161.

[40] D. Cremades, L. E. Ibanez and F. Marchesano, JHEP 0207 (2002) 009 hep-th/0201205.

[41] N. W. Kim, A. Pankiewicz, S. J. Rey and S. Theisen, Eur. Phys. J. C 25 (2002) 327 hep-th/0203080.

[42] A. Ishii, Prepared for School on Geometry and String Theory, Cambridge, England, 24 Mar - 20 Apr 2002

[43] S. Mukhi, M. Rangamani and E. P. Verlinde, JHEP 0205 (2002) 023 hep-th/0204147.

[44] P. Brax, A. Falkowski, Z. Lalak and S. Pokorski, Phys. Lett. B 538 (2002) 426 hep-th/0204195.

[45] T. J. Hollowood and S. P. Kumar, JHEP 0210 (2002) 077 hep-th/0206051.

[46] F. Denef, JHEP 0210 (2002) 023 hep-th/0206072.

[47] I. Frenkel, A. Malkin and M. Vybornov, arXiv:math-ph/0206012. 
[48] B. Feng, A. Hanany, Y. H. He and A. Iqbal, JHEP 0302 (2003) 056 hep-th/0206152.

[49] Z. Lalak, Prepared for 10th International Conference on Supersymmetry and Unification of Fundamental Interactions (SUSY02), Hamburg, Germany, 17-23 Jun 2002

[50] D. Berenstein and M. R. Douglas, hep-th/0207027.

[51] P. H. Frampton and P. Minkowski, hep-th/0208024.

[52] T. J. Hollowood and T. Kingaby, JHEP 0301 (2003) 005 hep-th/0210096.

[53] Y. H. He, JHEP 0302 (2003) 023 hep-th/0210127.

[54] S. Seki, Nucl. Phys. B 661 (2003) 257 hep-th/0212079.

[55] M. Ait Ben Haddou and E. H. Saidi, Phys. Lett. B 575 (2003) 100.

[56] P. H. Frampton, hep-th/0302057.

[57] R. Casero and E. Trincherini, JHEP 0309 (2003) 041 hep-th/0304123.

[58] J. Dai and Y. S. Wu, Phys. Lett. B 576 (2003) 209 hep-th/0306216.

[59] R. Casero and E. Trincherini, JHEP 0309 (2003) 063 hep-th/0307054.

[60] M. A. Benhaddou and E. H. Saidi, hep-th/0307103.

[61] K. Narayan and M. R. Plesser, hep-th/0309171.

[62] S. Mukhopadhyay and K. Ray, JHEP 0402 (2004) 070 hep-th/0309191.

[63] K. Narayan, Prepared for 3rd International Symposium on Quantum Theory and Symmetries (QTS3), Cincinnati, Ohio, 10-14 Sep 2003 
[64] J. Walcher, Prepared for 3rd International Symposium on Quantum Theory and Symmetries (QTS3), Cincinnati, Ohio, 10-14 Sep 2003

[65] A. Belhaj, hep-th/0310230.

[66] S. Chiantese, A. Klemm and I. Runkel, JHEP 0403 (2004) 033 hep-th/0311258.

[67] J. Dai and Y. S. Wu, Nucl. Phys. B 684 (2004) 75 hep-th/0312028.

[68] A. Belhaj and E. H. Saidi, Afr. J. Math. Phys. 1 (2004) 29.

[69] P. H. Frampton and T. Takahashi, Phys. Rev. D 70 (2004) 083530 hep-ph/0402119.

[70] J. Dai and Y. S. Wu, Nucl. Phys. B 708 (2005) 72 hep-th/0402201.

[71] H. Ita, H. Nieder, Y. Oz and T. Sakai, JHEP 0405 (2004) 058 hep-th/0403256.

[72] R. Ahl Laamara, M. Ait Ben Haddou, A. Belhaj, L. B. Drissi and E. H. Saidi, Nucl. Phys. B 702 (2004) 163 hep-th/0405222.

[73] D. Robles-Llana and M. Rocek, hep-th/0405230.

[74] E. Di Napoli, V. S. Kaplunovsky and J. Sonnenschein, JHEP 0406 (2004) 060 hep-th/0406122.

[75] N. Halmagyi, C. Romelsberger and N. P. Warner, Adv. Theor. Math. Phys. 10 (2006) 159 hep-th/0406143.

[76] N. Halmagyi, K. Pilch, C. Romelsberger and N. P. Warner, JHEP 0609 (2006) 063 hep-th/0406147.

[77] S. Franco and A. Hanany, JHEP 0503 (2005) 031 hep-th/0408016. 
[78] F. Fucito, J. F. Morales and R. Poghossian, JHEP 0410 (2004) 037 hep-th/0408090.

[79] D. Robles-Llana, hep-th/0411059.

[80] S. Benvenuti and A. Hanany, JHEP 0604 (2006) 032 hep-th/0411262.

[81] S. Benvenuti, S. Franco, A. Hanany, D. Martelli and J. Sparks, JHEP 0506 (2005) 064 hep-th/0411264.

[82] M. Bertolini, F. Bigazzi and A. L. Cotrone, JHEP 0412 (2004) 024 arXiv:hep-th/0411249].

[83] S. Benvenuti, A. Hanany and P. Kazakopoulos, JHEP 0507 (2005) 021 hep-th/0412279.

[84] E. Di Napoli, "Quiver gauge theories, chiral rings and random matrix models,"

[85] M. Billo, M. Frau, F. Lonegro and A. Lerda, JHEP 0505 (2005) 047 hep-th/0502084.

[86] A. Bergman, JHEP 0703 (2007) 112 hep-th/0502105.

[87] A. Hanany, P. Kazakopoulos and B. Wecht, JHEP 0508 (2005) 054 hep-th/0503177.

[88] A. D. Popov and R. J. Szabo, J. Math. Phys. 47 (2006) 012306 hep-th/0504025.

[89] S. Franco, A. Hanany, K. D. Kennaway, D. Vegh and B. Wecht, JHEP 0601 (2006) 096 hep-th/0504110.

[90] B. A. Burrington, J. T. Liu, M. Mahato and L. A. Pando Zayas, JHEP 0507 (2005) 019 hep-th/0504155.

[91] M. Bertolini, F. Bigazzi and A. L. Cotrone, Phys. Rev. D 72 (2005) 061902 |arXiv:hep-th/0505055|.

[92] S. Benvenuti and M. Kruczenski, JHEP 0604 (2006) 033 hep-th/0505206. 
[93] P. S. Aspinwall and L. M. Fidkowski, JHEP 0610 (2006) 047 hep-th/0506041.

[94] B. Szendroi, arXiv:math.ag/0506301.

[95] A. Takahashi, arXiv:math.ag/0506347.

[96] X. y. Zhu, arXiv:math.ag/0507316.

[97] S. Fujii and S. Minabe, arXiv:math.ag/0510455.

[98] A. Hanany and D. Vegh, hep-th/0511063.

[99] H. Kajiura, K. Saito and A. Takahashi, arXiv:math.ag/0511155.

[100] B. Feng, Y. H. He, K. D. Kennaway and C. Vafa, hep-th/0511287.

[101] M. Wijnholt, hep-th/0512122.

[102] N. J. Proudfoot and A. Bergman, arXiv:math.ag/0512166.

[103] D. Israel, JHEP 0604 (2006) 029 hep-th/0512166.

[104] Y. E. Antebi, Y. Nir and T. Volansky, Phys. Rev. D 73 (2006) 075009 hep-ph/0512211.

[105] A. Butti, D. Forcella and A. Zaffaroni, JHEP 0509 (2005) 018 arXiv:hep-th/0505220.

[106] A. Butti and A. Zaffaroni, Fortsch. Phys. 54 (2006) 309 hep-th/0512240.

[107] Y. Nakayama, Phys. Lett. B $\mathbf{6 3 6}$ (2006) 132 hep-th/0512280.

[108] K. Intriligator and N. Seiberg, JHEP 0602 (2006) 031 hep-th/0512347.

[109] A. Belhaj, J. Rasmussen, A. Sebbar and M. B. Sedra, J. Phys. A 39 (2006) 9339 hep-th/0512321. 
[110] T. W. Kephart, C. A. Lee and Q. Shafi, JHEP 0701, 088 (2007) arXiv:hep-ph/0602055.

[111] B. A. Burrington, J. T. Liu and L. A. Pando Zayas, Nucl. Phys. B 747 (2006) 436 hep-th/0602094.

[112] E. Di Napoli and P. H. Frampton, Phys. Lett. B638, 374 (2006). hep-th/0603065.

[113] I. Garcia-Etxebarria, F. Saad and A. M. Uranga, JHEP 0606 (2006) 055 hep-th/0603108.

[114] B. A. Burrington, J. T. Liu and L. A. Pando Zayas, Nucl. Phys. B 749 (2006) 245 hep-th/0603114.

[115] O. Lechtenfeld, A. D. Popov and R. J. Szabo, JHEP 0609 (2006) 054 hep-th/0603232.

[116] B. A. Burrington, J. T. Liu, M. Mahato and L. A. Pando Zayas, Nucl. Phys. B 757 (2006) 1 hep-th/0604092.

[117] J. Giedt, hep-lat/0605004.

[118] C. P. Herzog and R. L. Karp, hep-th/0605177.

[119] K. Ueda and M. Yamazaki, arXiv:math.ag/0605780.

[120] J. Park and W. Sim, JHEP 0610 (2006) 026 hep-th/0607075.

[121] A. Butti, D. Forcella and A. Zaffaroni, JHEP 0702 (2007) 081 hep-th/0607147.

[122] S. Benvenuti, B. Feng, A. Hanany and Y. H. He, hep-th/0608050.

[123] B. Florea, S. Kachru, J. McGreevy and N. Saulina, hep-th/0610003.

[124] T. Oota and Y. Yasui, Nucl. Phys. B 762 (2007) 377 hep-th/0610092. 
[125] D. Berenstein and S. Pinansky, Phys. Rev. D 75 (2007) 095009 hep-th/0610104.

[126] T. Volansky, AIP Conf. Proc. 903 (2007) 393 hep-ph/0611018.

[127] X. Zhu, Michigan Math. J. 54 (2006) 671.

[128] A. Butti, D. Forcella and A. Zaffaroni, JHEP 0706 (2007) 069 arXiv:hep-th/0611229|.

[129] R. Ahl Laamara, A. Belhaj, L. B. Drissi and E. H. Saidi, hep-th/0611289.

[130] B. A. Burrington, J. T. Liu and L. A. P. Zayas, hep-th/0701028.

[131] D. E. P. Diaconescu, R. Donagi and B. Florea, hep-th/0701104.

[132] D. Forcella, A. Hanany and A. Zaffaroni, arXiv:hep-th/0701236.

[133] Y. Imamura, H. Isono, K. Kimura and M. Yamazaki, hep-th/0702049.

[134] Y. E. Antebi and T. Volansky, hep-th/0703112.

[135] I. Garcia-Etxebarria, F. Saad and A. M. Uranga, arXiv:0704.0166 [hep-th].

[136] J. Evslin, C. Krishnan and S. Kuperstein, arXiv:0704.3484 [hep-th].

[137] D. L. Jafferis, arXiv:0705.2250 [hep-th].

[138] A. Butti, D. Forcella, A. Hanany, D. Vegh and A. Zaffaroni, arXiv:0705.2771 [hep-th].

[139] D. Forcella, arXiv:0705.2989 [hep-th]. 
[140] Z. Kakushadze, Phys. Lett. B 491, 317 (2000) hep-th/0008041.

[141] L. J. Dixon, J. A. Harvey, C. Vafa and E. Witten, Nucl. Phys. B 274, 285 (1986).

[142] L. J. Dixon, J. A. Harvey, C. Vafa and E. Witten, Nucl. Phys. B 261, 678 (1985).

[143] Z. Kakushadze and S. H. H. Tye, Phys. Lett. B 392, 335 (1997) hep-th/9609027.

[144] A. M. Uranga, JHEP 9901, 022 (1999) hep-th/9811004.

[145] K. Oh and R. Tatar, JHEP 9910, 031 (1999) hep-th/9906012.

[146] I. R. Klebanov and M. J. Strassler, of naked singularities," JHEP 0008, 052 (2000) hep-th/0007191.

[147] A. Hebecker and M. Ratz, Nucl. Phys. B 670, 3 (2003). hep-ph/0306049.

[148] S. Benvenuti and A. Hanany, JHEP 0508, 024 (2005). hep-th/0502043.

[149] Z. Kakushadze, Nucl. Phys. B 544, 265 (1999). hep-th/9808048.

[150] Z. Kakushadze, Phys. Rev. D 59, 045007 (1999). hep-th/9806091.

[151] A. Fayyazuddin and M. Spalinski, Nucl. Phys. B 535, 219 (1998). hep-th/9805096.

[152] Z. Kakushadze, Phys. Rev. D 58, 106003 (1998). hep-th/9804184.

[153] Z. Kakushadze, Nucl. Phys. B 529, 157 (1998). hep-th/9803214. 
[154] Z. Kakushadze, JHEP 0110, 031 (2001). hep-th/0109054.

[155] Z. Lalak, A. Lukas and B. A. Ovrut, Phys. Lett. B 425, 59 (1998) arXiv:hep-th/9709214.

[156] M. Faux, D. Lust and B. A. Ovrut, Nucl. Phys. B 554, 437 (1999) arXiv:hep-th/9903028.

[157] M. Faux, D. Lust and B. A. Ovrut, Nucl. Phys. B 589, 269 (2000) arXiv:hep-th/0005251].

[158] M. Faux, D. Lust and B. A. Ovrut, Int. J. Mod. Phys. A 18, 3273 (2003) arXiv:hep-th/0010087.

[159] M. Faux, D. Lust and B. A. Ovrut, Int. J. Mod. Phys. A 18, 2995 (2003) arXiv:hep-th/0011031.

[160] C. F. Doran, M. Faux and B. A. Ovrut, Adv. Theor. Math. Phys. 6, 329 (2003) arXiv:hep-th/0108078.

[161] G. Aldazabal, L. E. Ibanez, F. Quevedo and A. M. Uranga, JHEP 0008, 002 (2000) hep-th/0005067.

[162] Y. H. He, hep-th/0408142.

[163] S.L. Glashow, in Proceedings of the Fifth Workshop on Grand Unification, Editors: K.Kang, H. Fried and P.H. Frampton, World Scientific (1984). pages 88-94.

[164] J.C. Pati and A. Salam, Phys. Rev. D10, 275 (1974).

[165] T. Blazek, S. F. King and J. K. Parry, JHEP 0305, 016 (2003). hep-ph/0303192.

[166] J. B. Dent and T. W. Kephart, arXiv:0705.1995 [hep-ph].

[167] K. S. Babu, E. Ma and S. Willenbrock, Phys. Rev. D 69, 051301 (2004) hep-ph/0307380. 
[168] S. L. Chen and E. Ma, Mod. Phys. Lett. A 19, 1267 (2004) hep-ph/0403105.

[169] A. Demaria, C. I. Low and R. R. Volkas, Phys. Rev. D 72, 075007 (2005) [Erratum-ibid. D 73, 079902 (2006)] hep-ph/0508160.

[170] A. Demaria, C. I. Low and R. R. Volkas, Phys. Rev. D 74, 033005 (2006). hep-ph/0603152.

[171] A. Demaria and K. L. McDonald, Phys. Rev. D 75, 056006 (2007) hep-ph/0610346.

[172] K. S. Babu, T. W. Kephart and H. Pas, arXiv:0709.0765 [hep-ph].

[173] P.H. Frampton and T.W. Kephart, Int. J. Mod. Phys. A19, 593 (2004). hep-th/0306207.

[174] P.H. Frampton and W. F. Shively, Phys. Lett. B454, 49 (1999). hep-th/9902168.

[175] P.H. Frampton and C. Vafa. hep-th/9903226.

[176] P.H. Frampton, Phys. Rev. D60, 085004 (1999). hep-th/9905042.

[177] P.H. Frampton, Phys. Rev. D60, 121901 (1999). hep-th/9907051.

[178] P.H. Frampton and T.W. Kephart, Phys. Lett. B485, 403 (2000). hep-th/9912028.

[179] P.H. Frampton, J. Math. Phys. 42, 2915 (2001). hep-th/0011165.

[180] P.H. Frampton and T.W. Kephart, Phys. Rev. D64, 086007 (2001). hep-th/0011186.

[181] P.H. Frampton, R.N. Mohapatra and S. Suh, Phys. Lett. B520, 331 (2001). hep-ph/0104211. 
[182] S. Kachru and E. Silverstein, Phys. Rev. Lett. 80, 4855 (1998). hep-th/9802183.

[183] T. W. Kephart and H. Päs, Phys. Lett. B 522, 315 (2001) hep-ph/0109111.

[184] P.H. Frampton, Mod. Phys. Lett. A18, 1377 (2003). hep-ph/0208044.

[185] P.H. Frampton, R.M. Rohm and T. Takahashi, Phys. Lett. B570, 67 (2003). hep-ph/0302074.

[186] P.H. Frampton and T.W. Kephart, Phys. Lett. B585, 24 (2004). hep-th/0306053.

[187] A. Lawrence, N. Nekrasov and C. Vafa, Nucl. Phys. B533, 199 (1998). hep-th/9803015.

[188] T.W. Kephart and H. Paes, Phys. Lett. B522, 315 (2001). hep-ph/0109111.

[189] P. H. Frampton, hep-ph/0208044.

[190] T.W. Kephart and H. Paes, Phys. Rev. D70, 086009 (2004). hep-ph/0402228.

[191] A. G. Pickering, J. A. Gracey and D. R. Jones, Phys. Lett. B 510, 347 (2001) [Phys. Lett. B 512, 230 (2001)] hep-ph/0104247.

[192] S. Kachru and E. Silverstein, Phys. Rev. Lett. 80, 4855 (1998) hep-th/9802183.

[193] T. W. Kephart and H. Päs, hep-ph/0102243.

[194] P.H. Frampton and T.W. Kephart, Int. J. Mod. Phys. A10, 4689 (1995).

[195] D.E. Littlewood, it The Theory of Group Characters and Matrix Representations of Groups (Oxford 1940). 
[196] M. Hamermesh, Group Theory and Its Applications to Physical Problems (Addison-Wesley, 1962).

[197] J.S. Lomont, Applications of Finite Groups (Academic, 1959), reprinted by Dover (1993).

[198] A.D. Thomas and G.V. Wood, Group Tables (Shiva, 1980).

[199] I. Antoniadis, Phys. Lett. B246, 377 (1990).

[200] I. Antoniadis and K. Benakli, Phys. Lett. B326, 69 (1994).

[201] I. Antoniadis, K. Benakli and M. Quiros, Phys. Lett. B331, 313 (1994).

[202] J. D. Lykken, Phys. Rev. D54, 3693 (1996).

[203] I. Antoniadis, N. Arkani-Hamed, S. Dimopoulos and G. Dvali, Phys. Lett. B436, 257 (1998).

[204] I. Antoniadis, S. Dimopoulos, A. Pomarol and M. Quiros, Nucl. Phys. B544, 503 (1999).

[205] K. Dienes, E. Dudas and T. Gherghetta, Phys. Lett. B436, 55 (1998); Nucl. Phys. 537, 47 (1999).

[206] K. Dienes, E. Dudas and T. Gherghetta, Phys. Lett. B436, 55 (1998). Nucl. Phys. B543, 387 (1999).

[207] P.H. Frampton and A. Rasin, Phys. Lett. 460B, 313 (1999).

[208] D. Ghilencea and G.G. Ross, Phys. Lett. B442, 165 (1998).

[209] C.D. Carone, Phys. Lett. B454, 70 (1999).

[210] C. Bachas, JHEP 981, 023 (1998).

[211] G. Shiu and S.-H. H. Tye, Phys. Rev. D58, 106007 (1998).

[212] Z. Kakushadze and S.H. Tye, Nucl. Phys. B548, 180 (1999). 
[213] Z. Kakushadze, Nucl. Phys. B551, 549 (1999).

[214] N. Arkani-Hamed, S. Dimopoulos and G. Dvali, Phys. Lett 429, 506 (1998).

[215] K.S. Babu, X.G. He and S. Pakvasa, Phys. Rev. D33, 763 (1986).

[216] X. Calmet, P.H. Frampton and R.M. Rohm, Phys. Rev. D72, 055005 (2005). hep-th/0412176

[217] P.H. Frampton, Mod. Phys. Lett. A21, 893 (2006). hep-th/0511265.

[218] L. Brink, J. Scherk and J.H. Schwarz, Nucl. Phys. B121, 77 (1977).

[219] K.R. Dienes, Nucl. Phys. B429, 533 (1994). hep-th/9402006.

[220] Y. Nambu, Phys. Lett. B26, 626 (1968).

[221] P.H. Frampton, Mod. Phys. Lett. 22A, 931 (2007). astro-ph/0607391.

[222] H. Georgi and S.L. Glashow, Phys. Rev. Lett. 32, 438 (1974);

[223] H. Georgi, H.R. Quinn and S. Weinberg, Phys. Rev. Lett. 33, 451 (1974).

[224] P.H. Frampton, Mod. Phys. Lett. 22A, 347 (2007). hep-ph/0610116.

[225] J. B. Dent and T. W. Kephart, arXiv:0704.1451 [hep-ph], Phys. Rev D, to appear.

[226] A. Adams and E. Silverstein, Phys. Rev. D64, 086001 (2001). hep-th/0103220.

[227] A. Adams, J. Polchinski and E. Silverstein, JHEP 0110:029 (2001). hep-th/0108075. 
[228] M. Bershadsky and A. Johansen, Nucl Phys. B536, 141 (1998). hep-th/9803249.

[229] M. Bershadsky, Z. Kakushadze and C. Vafa, Nucl. Phys. B523, 59 (1998). hep-th/9803076.

[230] A. Dymarsky, I.R. Klebanov and R. Roiban, JHEP 0508:011 (2005). hep-th/0505099.

[231] A. Dymarsky, I.R. Klebanov and R. Roiban, JHEP 0511:038 (2005). hep-th/0509132. 